\title{
Long-rising Type II supernovae from Palomar Transient Factory and Caltech Core-Collapse Project
}

\author{
F. Taddia ${ }^{1}$, J. Sollerman ${ }^{1}$, C. Fremling ${ }^{1}$, K. Migotto ${ }^{1}$, A. Gal-Yam² ${ }^{2}$, S. Armen ${ }^{3}$, G. Duggan ${ }^{4}$, M. Ergon ${ }^{1}$, \\ A. V. Filippenko ${ }^{5}$, C. Fransson ${ }^{1}$, G. Hosseinzadeh ${ }^{6,7}$, M. M. Kasliwal ${ }^{8}$, R. R. Laher ${ }^{9}$, G. Leloudas ${ }^{2,10}$, D. C. Leonard ${ }^{3}$, \\ R. Lunnan ${ }^{4}$, F. J. Masci ${ }^{11}$, D.-S. Moon ${ }^{12}$, J. M. Silverman ${ }^{13}$, and P. R. Wozniak ${ }^{14}$ \\ 1 The Oskar Klein Centre, Department of Astronomy, Stockholm University, AlbaNova, 10691 Stockholm, Sweden \\ e-mail: francesco.taddia@astro.su.se \\ 2 Department of Particle Physics \& Astrophysics, Weizmann Institute of Science, 76100 Rehovot, Israel \\ 3 Department of Astronomy, San Diego State University, San Diego, CA 92182-1221, USA \\ 4 Astronomy Department, California Institute of Technology, Pasadena, California 91125, USA \\ 5 Department of Astronomy, University of California, Berkeley, CA 94720-3411, USA \\ 6 Las Cumbres Observatory Global Telescope, 6740 Cortona Dr, Suite 102, Goleta, CA 93117, USA \\ 7 Department of Physics, University of California, Santa Barbara, CA 93106-9530, USA \\ 8 The Observatories, Carnegie Institution for Science, 813 Santa Barbara Street, Pasadena, CA 91101, USA \\ 9 Spitzer Science Center, California Institute of Technology, M/S 314-6, Pasadena, CA 91125, USA \\ 10 Dark Cosmology Centre, Niels Bohr Institute, University of Copenhagen, Juliane Maries Vej 30, 2100 Copenhagen, Denmark \\ 11 Infrared Processing and Analysis Center, California Institute of Technology, MS 100-22, Pasadena, CA 91125, USA \\ 12 Department of Astronomy and Astrophysics, University of Toronto, Toronto, ON M5S 3H4, Canada \\ 13 Department of Astronomy, University of Texas, Austin, TX 78712, USA \\ ${ }^{14}$ Los Alamos National Laboratory, MS D436, Los Alamos, NM 87545, USA
}

Received 23 November 2015 / Accepted 23 January 2016

\section{ABSTRACT}

\begin{abstract}
Context. Supernova (SN) 1987A was a peculiar hydrogen-rich event with a long-rising ( 84 d) light curve, stemming from the explosion of a compact blue supergiant star. Only a few similar events have been presented in the literature in recent decades.

Aims. We present new data for a sample of six long-rising Type II SNe (SNe II), three of which were discovered and observed by the Palomar Transient Factory (PTF) and three observed by the Caltech Core-Collapse Project (CCCP). Our aim is to enlarge this small family of long-rising SNe II, characterizing their differences in terms of progenitor and explosion parameters. We also study the metallicity of their environments.

Methods. Optical light curves, spectra, and host-galaxy properties of these SNe are presented and analyzed. Detailed comparisons with known SN 1987A-like events in the literature are shown, with particular emphasis on the absolute magnitudes, colors, expansion velocities, and host-galaxy metallicities. Bolometric properties are derived from the multiband light curves. By modeling the earlytime emission with scaling relations derived from the SuperNova Explosion Code (SNEC) models of MESA progenitor stars, we estimate the progenitor radii of these transients. The modeling of the bolometric light curves also allows us to estimate other progenitor and explosion parameters, such as the ejected ${ }^{56} \mathrm{Ni}$ mass, the explosion energy, and the ejecta mass.

Results. We present PTF12kso, a long-rising SN II that is estimated to have the largest amount of ejected ${ }^{56} \mathrm{Ni}$ mass measured for this class. PTF09gpn and PTF12kso are found at the lowest host metallicities observed for this SN group. The variety of early lightcurve luminosities depends on the wide range of progenitor radii of these $\mathrm{SNe}$, from a few tens of $R_{\odot}$ (SN 2005ci) up to thousands (SN 2004ek) with some intermediate cases between $100 R_{\odot}$ (PTF09gpn) and $300 R_{\odot}(\mathrm{SN} 2004 \mathrm{em})$.

Conclusions. We confirm that long-rising SNe II with light-curve shapes closely resembling that of SN 1987A generally arise from blue supergiant (BSG) stars. However, some of them, such as SN 2004em, likely have progenitors with larger radii $\left(\sim 300 R_{\odot}\right.$, typical of yellow supergiants) and can thus be regarded as intermediate cases between normal SNe IIP and SN 1987A-like SNe. Some extended red supergiant (RSG) stars such as the progenitor of SN 2004ek can also produce long-rising SNe II if they synthesized a large amount of ${ }^{56} \mathrm{Ni}$ in the explosion. Low host metallicity is confirmed as a characteristic of the SNe arising from compact BSG stars.
\end{abstract}

Key words. supernovae: general - Galaxy: abundances

\section{Introduction}

Supernova (SN) 1987A marked the explosion of a blue supergiant (BSG) star (Sanduleak $-69^{\circ} 202$, Gilmozzi et al. 1987), and it showed a peculiar long-rising light curve (84 days from explosion to peak) and a hydrogen-rich (Type II SN) spectrum. Given its extraordinary proximity (merely $50 \mathrm{kpc}$, in the Large Magellanic Cloud-LMC), SN 1987A is to date the best-studied
SN. It changed our understanding of SN explosions (see the reviews by Arnett et al. 1989 and McCray 1993), and it continues to trigger scientific activity; it is the only SN that can be spatially resolved in the optical and was the first one whose transition to a young SN remnant could be observed (Larsson et al. 2011).

In the last decade, a handful of SNe resembling SN 1987A have been presented in the literature. Most of these SN 1987Alike SNe were collected by Pastorello et al. (2012). The 
best-observed objects are SNe 1998A (Pastorello et al. 2005), 2000cb (Kleiser et al. 2011), 2006V, 2006au (Taddia et al. 2012), and 2009E (Pastorello et al. 2012). Well-sampled optical light curves of the long-rising ( $>40$ d) SNe II 2004ek, 2004em, and 2005ci by the Caltech Core-Collapse Project (CCCP; Gal-Yam et al. 2007) were shown by Arcavi et al. (2012), and here we present their full datasets, including unpublished spectroscopy. González-Gaitán et al. (2015) also discussed slow risers in their sample of SNe II. Some of their SNe lack spectral classifications and might be of Type IIn; however, at least one spectroscopically confirmed SN 1987A-like event is presented (SNLS-07D2an), with a rise time of $>60 \mathrm{~d}$. The first SN 1987A-like event was likely SN 1909A (Young \& Branch 1988), but for this object there is no spectral classification.

The analysis of the light curves and spectra of the SNe discussed by Pastorello et al. (2012) led to the conclusion that they arise from the explosions of compact $\left(R<100 R_{\odot}\right)$ BSG stars, with initial masses of about $M_{\text {ZAMS }}=20 M_{\odot}$, explosion energies of a few $10^{51} \mathrm{erg}$, and ${ }^{56} \mathrm{Ni}$ masses of $\sim 10^{-1} M_{\odot}$.

As BSG stars are not expected to explode in the standard stellar evolution models, following the explosion of Sanduleak $-69^{\circ} 202$ there were many attempts to explain this surprising fact (see Podsiadlowski 1992, for a review). Models showed that a low metallicity (like that measured in the LMC) and/or fast rotation could make a single $M_{\text {ZAMS }}=20 M_{\odot}$ star explode in the blue part of the Hertzsprung-Russell (HR) diagram. We (Taddia et al. 2013) presented a detailed study of the metallicity at the locations of the known SN 1987A-like events, finding that these $\mathrm{SNe}$ indeed tend to occur at lower (LMC-like) metallicity than normal SNe IIP. Alternatively, a binary scenario may also explain BSG explosions, and in the specific case of SN 1987A (Podsiadlowski 1992) it may also explain the complex shape of the circumstellar medium (CSM).

Given the rarity of long-rising SNe II, it is important to extend the number of well-observed objects, in order to study the variety of their properties. The observations from the Palomar Transient Factory (PTF; Rahmer et al. 2008; Rau et al. 2009) and from the CCCP that we present here are therefore significant additions to this $\mathrm{SN}$ subgroup.

The PTF discoveries are particularly important as they are not biased toward bright (i.e., metal-rich) host galaxies (Arcavi et al. 2010). Our small PTF sample of SN 1987A-like events allowed us to find these $\mathrm{SNe}$ at unprecedented low metallicity, with important implications for the progenitor scenario. We notice that other rare and peculiar core-collapse (CC) SNe have been observed in low or very low metallicity environments (e.g., the Type Ic-BL PTF12gzk; Ben-Ami et al. 2012). Other recent examples are the class of hydrogen-poor superluminous $\mathrm{SNe}$ (SLSNe; Quimby et al. 2011; Gal-Yam 2012; Lunnan et al. 2014; Leloudas et al. 2015), and that of the SN impostors (Taddia et al. 2015a).

The PTF survey and its continuation (the intermediate PTF, iPTF; Kulkarni 2013) not only extends the domain of SN discovery to low-luminosity hosts, but thanks to the high cadence allows regular discoveries of very young transients (e.g., iPTF13ast; Gal-Yam et al. 2014). Indeed, some of our PTF (but also CCCP) objects were likely discovered and observed soon after explosion. Therefore, some of them show possible signatures of the shock-breakout cooling tail in their optical light curves (Chevalier 1992; Chevalier \& Fransson 2008; Rabinak \& Waxman 2011; Piro \& Nakar 2013). This feature is rarely observed and contains important information to understand the nature of the SN progenitor. First, it allows an estimate of the explosion epoch with small uncertainty, which is crucial for
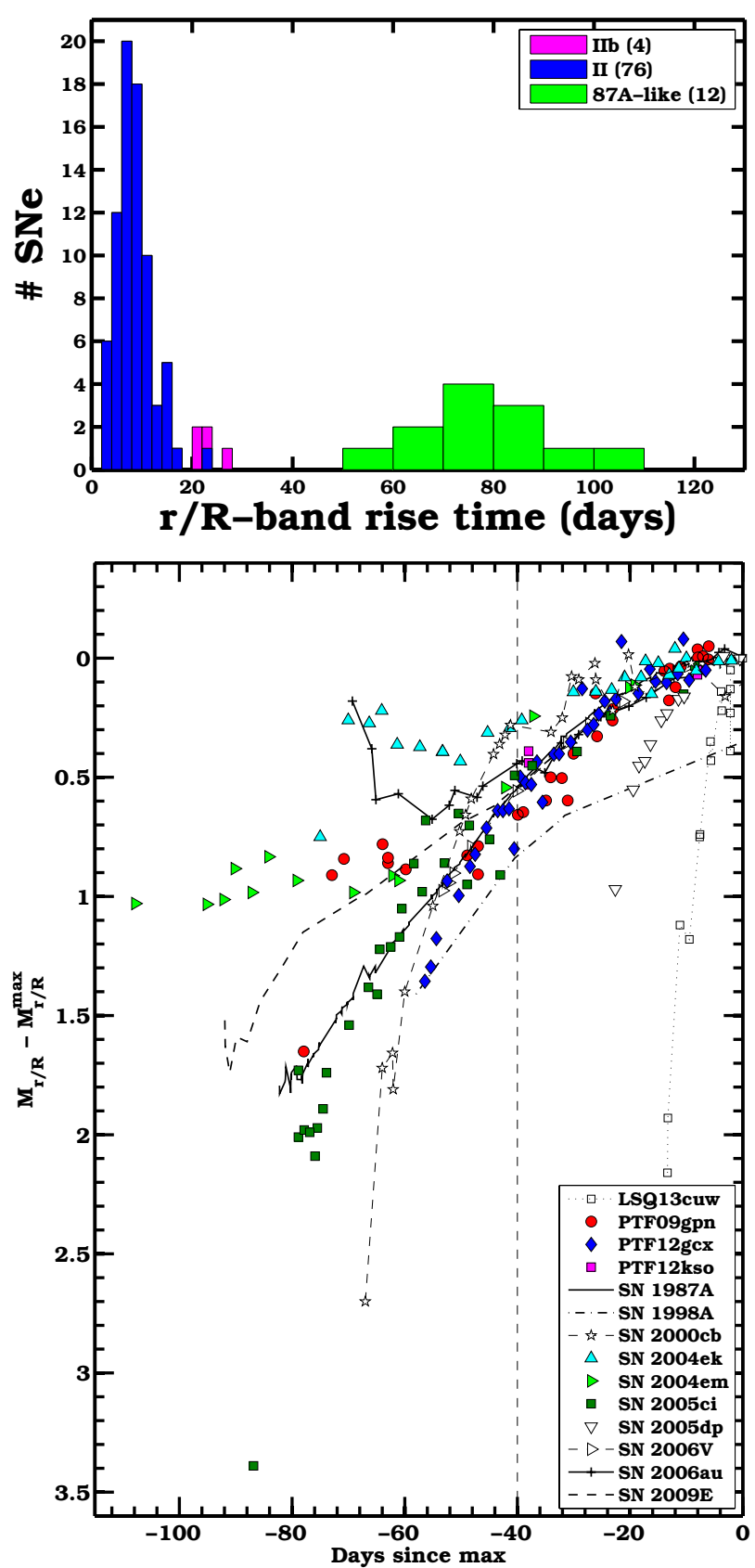

Fig. 1. Top panel: distribution of $r / R$-band rise times for SNe IIP, IIL, IIb, and SN 1987A-like SNe from the literature (Gall et al. 2015; Taddia et al. 2015a) and from this work. (See Filippenko 1997, for a review of SN classification.) We define as long-rising SNe II or SN 1987A-like events those with rise time $>40 \mathrm{~d}$. These SNe are powered mainly by radioactive decay at peak, whereas normal SN II peaks are dominated by shock energy. Bottom panel: $r / R$-band light curves of slow-rising $\mathrm{SNe}$ II (from this work, when colored, and from the literature), aligned to the peak epoch and scaled to match their peaks. All of them show rise times $>40 \mathrm{~d}$, but display a variety of shapes before peak. We also show SN 2005dp (Arcavi et al. 2012) and LSQ13cuw (Gall et al. 2015), which have relatively long rise times compared to normal SNe IIP, but still $<40 \mathrm{~d}$.

deriving the properties of the progenitor from light-curve and spectral modeling. In addition, especially for objects that are radioactively powered at peak (like our long-rising SNe II), the modeling of the early-time light curve can constrain the radius of the SN progenitor. 
Table 1. PTF and CCCP sample of SN 1987A-like SNe.

\begin{tabular}{|c|c|c|c|c|c|c|c|c|c|}
\hline$S N$ & $\begin{array}{l}\alpha(\mathrm{J} 2000) \\
\text { (hh:mm:ss) }\end{array}$ & $\begin{array}{l}\delta(\mathrm{J} 2000) \\
(\mathrm{dd}: \mathrm{mm}: \mathrm{ss})\end{array}$ & Galaxy & $\begin{array}{c}\text { Galaxy } \\
\text { Type }\end{array}$ & Redshift & $\begin{array}{l}\text { Distance } \\
(\mathrm{Mpc})\end{array}$ & $\begin{array}{l}A_{V}^{\mathrm{MW}} \\
(\mathrm{mag})\end{array}$ & $\begin{array}{c}A_{V}^{\text {host }} \\
(\mathrm{mag})\end{array}$ & $\begin{array}{c}E W(\mathrm{Na} \text { I D })_{\mathrm{h}} \\
(\AA)\end{array}$ \\
\hline $2004 \mathrm{ek}$ & 01:09:58.51 & $+32: 22: 47.7$ & UGC 724 & $S$ & 0.017275 & $75.3 \pm 2.1$ & 0.183 & $0.347 \pm 0.015$ & $0.70 \pm 0.03$ \\
\hline $2004 \mathrm{em}$ & $19: 31: 31.11$ & $+35: 52: 15.7$ & IC 1303 & SAc & 0.014894 & $59.6 \pm 1.7$ & 0.298 & $\ldots$ & n.d. \\
\hline $2005 \mathrm{ci}$ & $14: 34: 44.88$ & $+48: 40: 19.8$ & NGC 5682 & $\mathrm{SB}(\mathrm{s}) \mathrm{b}$ & 0.007581 & $36.3 \pm 13.8$ & 0.089 & $\ldots$ & n.d. \\
\hline PTF09gpn & $03: 43: 43.26$ & $-17: 08: 43.1$ & Anon. & $\ldots$ & 0.0154 & $66.3 \pm 1.2$ & 0.453 & $\ldots$ & n.d. \\
\hline PTF12gcx & $15: 44: 17.32$ & $+09: 57: 43.1$ & SDSS J154417.02+095743.8 & $\ldots$ & 0.045041 & $198.3 \pm 3.7$ & 0.140 & $\ldots$ & n.d. \\
\hline PTF12kso & 04:36:10.89 & $-05: 04: 53.3$ & Anon. & $\ldots$ & 0.030 & $130.6 \pm 2.4$ & 0.184 & $\ldots$ & n.d. \\
\hline
\end{tabular}

Notes. MW extinctions are obtained via NED (Fitzpatrick 1999; Schlafly \& Finkbeiner 2011). Redshifts are from NED except for PTF09gpn and PTF12kso, where we measured it from the narrow lines of their host-galaxy spectra. Distances are redshift-independent for the three CCCP SNe, and obtained from the redshift for the three PTF SNe (assuming WMAP5 cosmological parameters; Komatsu et al. 2009). PTF12kso is also known as SN 2012gg, CSS J043611.05-050452.1, and LSQ12fjm (Sternberg et al. 2012).

Here we present new observations of six long-rising SNe II, which appear rather similar to SN 1987A. The paper is structured as follows. In Sect. 2 we describe the SN sample, and in Sect. 3 we discuss data acquisition and reduction. Section 4 includes the analysis of the host galaxies. We describe and analyze SN photometry and spectroscopy in Sects. 5 and 6, respectively. Bolometric properties are derived in Sect. 7. Data are modeled in Sect. 8, in order to derive the progenitor parameters. We discuss the results in Sect. 9 and give our conclusions in Sect. 10.

\section{The supernova sample}

We define a SN II as "long-rising" based on the epoch of the (second) peak of its $r / R$-band light curve. In Fig. 1 (top panel) we show the distribution of $r / R$-band rise times of SNe II, with data for normal SNe II (IIP and IIL) and SNe IIb from Gall et al. (2015), Rubin et al. (2015), and Taddia et al. (2015a), as well as data for long-rising SNe II from the light curves discussed in Sect. 5 and displayed in the bottom panel of Fig. 1. SNe showing rise times $>40 \mathrm{~d}$ are considered "long risers" in this work. Interestingly, among SNe II (excluding SNe IIn, which are powered by CSM interaction), there is a lack of SNe rising in 30$50 \mathrm{~d}$. This is likely due to the fact that the explosion energy and the ejecta mass (up to $\sim 25 M_{\odot}$ ) of SNe II appear to be linearly correlated (Utrobin et al. 2010), and their ratios imply long rise times for compact progenitor stars (see Eq. (2)).

We include in our definition of slow risers only those $\mathrm{SNe}$ with rise times $>40 \mathrm{~d}$, to be sure to include only objects that are mainly powered by radioactive decay at peak, like SN 1987A. In SNe IIP and IIL, a rise of only a few weeks in the optical could instead be due to a temperature effect, with most of the flux in the ultraviolet (UV) part of the spectrum until $\sim 20-25 \mathrm{~d}$. This is why we exclude from our sample another CCCP SN II with relatively slow rise (24 d), SN 2005dp (Arcavi et al. 2012). The rising part of the $R$-band light curve of this transient is shown in the bottom panel of Fig. 1, and it is clearly distinct from the family of slow risers resembling SN 1987A. For SNe IIb, the peak is powered by radioactive decay as in SN 1987A, but their shorter rise times are due to the lower ejecta mass as compared to SN 1987A-like $\mathrm{SNe}$. SNe IIb have lost their $\mathrm{H}$ envelopes almost entirely prior to exploding.

As shown in the bottom panel of Fig. 1, the slow risers can exhibit a variety of light-curve shapes at early epochs. Their rise can be only a few tenths of a magnitude from the early minimum to the second peak (e.g., PTF09gpn), or it can be several magnitudes (e.g., SN 1987A). We include both of these types of objects in the category of long-rising SNe II or SN 1987A-like events. Their common feature is the late-time peak, which we will show also appears in their bolometric light curves; it is therefore likely to be powered mainly by the decay chain of ${ }^{56} \mathrm{Ni}$. We will propose that the variety of the early-time light curves is a result of the explosion of progenitors with different radii (see Sect. 8.3 and the results by Young 2004).

Here we present data for six SNe II with long-rising light curves. Three of them were discovered and followed by PTF (PTF09gpn, PTF12gcx, PTF12kso), and three were observed by CCCP (SNe 2004ek, 2004em, 2005ci). For the PTF objects we present unpublished light curves and spectra. For the CCCP objects we present unpublished spectra and make use of the light curves already included in Arcavi et al. (2012). In Table 1 the basic information on the targets is given, including SN coordinates, host-galaxy name and type, redshift, distance, and extinction. The redshifts are obtained from $\mathrm{NED}^{1}$ for the CCCP SNe and for PTF12gcx, and from the host-galaxy emission lines in the case of the other two PTF objects. We adopted redshift-independent distances (from NED) when available (i.e., in the case of the CCCP targets), whereas we used the known redshift and the WMAP5 cosmological parameters $\left(H_{0}=70.5 \mathrm{~km} \mathrm{~s}^{-1} \mathrm{Mpc}^{-1}\right.$, $\Omega_{\mathrm{M}}=0.27, \Omega_{\Lambda}=0.73$; Komatsu et al. 2009) to compute the distances for the hosts without redshift-independent estimates (i.e., for the PTF SNe). The Galactic extinction is obtained from NED (Schlafly \& Finkbeiner 2011), the host-galaxy extinction (non-negligible only in the case of SN 2004ek) is obtained from the NaI D equivalent width (EW) via the relation $E(B-V)=0.16 \mathrm{EW}(\mathrm{Na}$ I D) (Taubenberger et al. 2006) and assuming $R_{V}=3.1$. Here we neglect the correction for time dilation as these $\mathrm{SNe}$ are all in low-redshift galaxies.

\section{Data acquisition and reduction}

\subsection{Discovery and explosion epochs}

In Table 2 we report the last nondetection date and limiting magnitude, the discovery date and magnitude (along with their references), and the assumed explosion epoch for each SN. It is important to constrain the explosion date for each SN, in order to compare the different $\mathrm{SNe}$ at similar phases and to derive the progenitor parameters. The last nondetection and the discovery

1 The NASA/IPAC Extragalactic Database, http://ned.ipac. caltech.edu 
A\&A 588, A5 (2016)

Table 2. Discovery data for the PTF and CCCP sample of SN 1987A-like SNe.

\begin{tabular}{|c|c|c|c|c|c|c|c|}
\hline$S N$ & $\begin{array}{l}\text { Discovery }^{a} \\
\text { source }\end{array}$ & $\begin{array}{l}\text { Classification }^{a} \\
\text { source }\end{array}$ & $\begin{array}{l}\text { Last nondetection } \\
\text { JD-2 } 450000\end{array}$ & $\begin{array}{l}\text { Limit } \\
\text { mag }\end{array}$ & $\begin{array}{c}\text { Discovery } \\
\text { JD-2 } 450000\end{array}$ & $\begin{array}{l}\text { Discovery } \\
\text { mag }\end{array}$ & $\begin{array}{l}\text { Explosion date } \\
\text { JD-2450000 }\end{array}$ \\
\hline $2004 \mathrm{ek}$ & IAUC 8405 & CBET 86 & 3242.5 & 19.5 & 3258.4730 & 17.1 & $3250.5 \pm 8.0$ \\
\hline $2004 \mathrm{em}$ & CBET 82 & IAUC 8411 & 3214.5 & 19.0 & 3263.4680 & 17.5 & $3263.3_{-7.10}^{+0.168}$ \\
\hline $2005 \mathrm{ci}$ & CBET 168 & CBET 169/Kleiser et al. (2011) & 3523.81 & 19.0 & 3523.86 & 20.0 & $3523.69_{-12.03}^{+0.17}$ \\
\hline PTF09gpn & PTF (Arcavi et al. 2010) & PTF (Arcavi et al. 2010) & $5127.91(\mathrm{P} 48)$ & $21.00(\mathrm{P} 48)$ & $5137.77(\mathrm{P} 48)$ & $20.30(\mathrm{P} 48)$ & $5137.23_{-0.05}^{+0.05}$ \\
\hline PTF12gcx & PTF (ATel 4293) & PTF (ATel 4293) & 6085.39 (P48) & $20.93(\mathrm{P} 48)$ & $6085.84(\mathrm{P} 48)$ & $21.14(\mathrm{P} 48)$ & $6085.72_{-0.40}^{+0.12}$ \\
\hline PTF12kso & PTF / CBET 3296,1 & PTF / CBET 3296.2 & $6153.00(\mathrm{P} 48)$ & $20.85(\mathrm{P} 48, g)$ & $6198.98(\mathrm{P} 48)$ & $18.41(\mathrm{P} 48)$ & $6176.0 \pm 23.0$ \\
\hline
\end{tabular}

Notes. Magnitudes are unfiltered or $r$ band except for the limiting magnitude of PTF12kso. For PTF12kso there is a nondetection at JD= 2456169.5 by LSQ (Aug. 30), which is later than the last PTF nondetection, but the limiting magnitude is unknown. LSQ discovered it at $\mathrm{JD}=2456202.7324$, later than PTF. For SN 2005ci the explosion date $\left(3522.3_{-30}^{+1.5}\right)$ derived from the expanding photospheric method (EPM; see Jones et al. 2009) using the VI bands and the first two spectra matches that derived from the PL fit which we adopted. We notice that the explosion date of SN 2005ci occurs before the last nondetection, but the magnitude limit at this phase was not deep enough (19 mag) to detect SN 2005ci.

References. ${ }^{(a)}$ References: IAUC 8405: Monard et al. (2004); CBET 86: Modjaz et al. (2004); CBET 82: Armstrong (2004); IAUC 8411: Filippenko et al. (2004); CBET 168: Madison \& Li (2005); CBET 169: Modjaz et al. (2005); ATel 4293: Gal-Yam et al. (2012); CBET 3296, 1: Mahabal et al. (2012); CBET 3296, 2: Tomasella et al. (2012).

epochs can be used to constrain the explosion epoch, if they occur close in time and the nondetection magnitude limit is sufficiently deep. Alternatively, a power-law (PL) fit to the early-time light curves can be used (e.g., Cao et al. 2013).

For SNe 2004em, 2005ci, PTF09gpn, and PTF12gcx, we used a PL fit to the early light curves to estimate the explosion epoch. For SN 2004em we fit the first six epochs of the bolometric light curve (see Sect. 7). For SN 2005ci, PTF09gpn, and PTF12gcx, we fit the first 11,3, and 7 epochs of the $r / R$ band light curves (see Sect. 5), respectively. The uncertainty in the explosion date is determined by the fit uncertainty and/or by the difference between the discovery and the last nondetection epochs.

For SN 2004ek we derive the explosion date from the average between discovery and the last nondetection, and the uncertainty from the interval between these two epochs. We adopt the same method for PTF12kso as we do not have observations of this SN at early times that would allow us to fit a PL.

In the rest of the paper the phase of each object will be defined in days since explosion.

\subsection{CCCP supernova data}

The CCCP BVRI SN light curves were obtained from Arcavi et al. (2012) via Wiserep (Yaron \& Gal-Yam 2012). Additional data for SNe 2004ek and 2005ci were obtained from Kleiser et al. (2011, unfiltered) and Tsvetkov (2008, BVRI), respectively. The $B V R I$ photometry from Tsvetkov (2008) was shifted by $+0.1,+0.2,+0.2$, and +0.1 mag (respectively), in order to match the CCCP photometry of SN 2004ek. We report all the magnitudes in Table A.1.

Five spectra of each of SNe 2004ek and 2004em were obtained with the Double Spectrograph (DBSP; Oke \& Gunn 1982) mounted on the 200-inch Hale telescope at Palomar Observatory (P200), the Low Resolution Imaging Spectrometer (LRIS; Oke et al. 1995) mounted on the Keck 1 10-m telescope, and the Kast spectrograph (Miller \& Stone 1993) on the 3-m Shane reflector at Lick Observatory. Three spectra of SN 2005ci were obtained with P200+DBSP. For a detailed log of the CCCP spectral observations, see Table A.3. The spectra were reduced in a standard manner, including (i) bias and flatfield corrections; (ii) onedimensional (1D) spectral extraction from the 2D frames after tracing and choosing proper apertures and background regions;

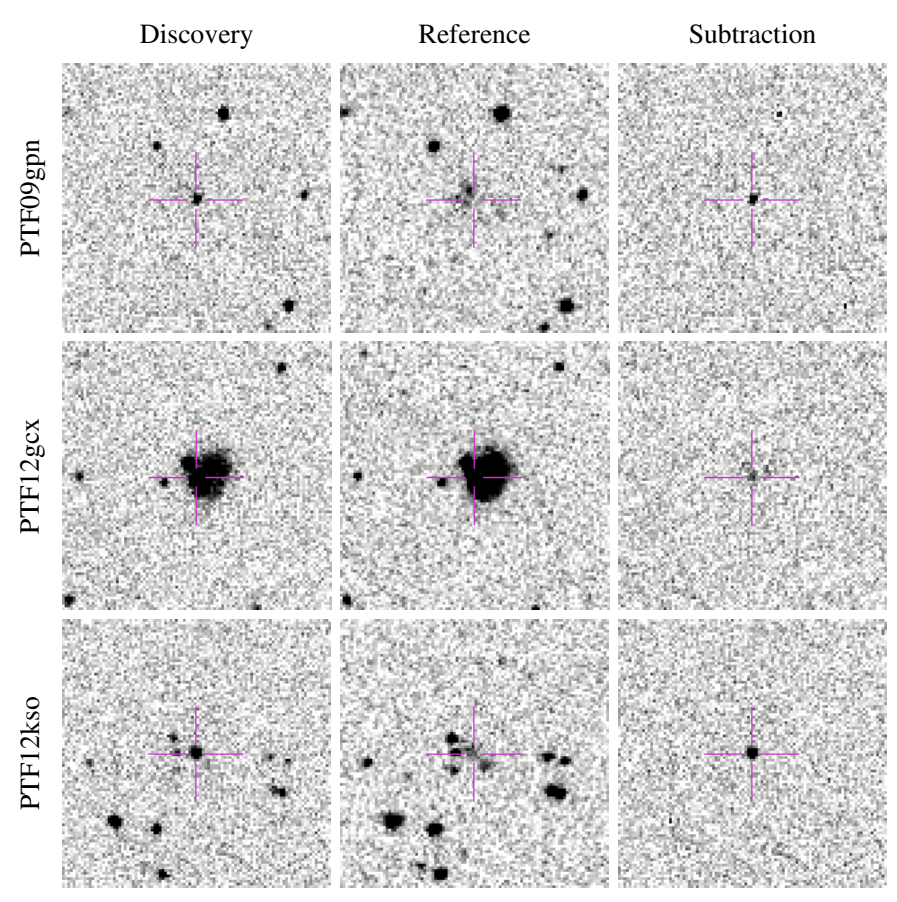

Fig. 2. Discovery, reference, and subtracted P48 $r$-band images for the three PTF SNe. North is up and east is to the left. The field of view is $1.67^{\prime} \times 1.67^{\prime}$.

(iii) wavelength calibration with spectra of calibration lamps; and (iv) flux calibration with spectrophotometric standard stars observed during the same night.

\subsection{PTF supernova data}

In Fig. 2 we show the discovery images, along with reference and subtracted images, of the three PTF SNe. For these SNe $r$-band photometry was obtained with the 48-inch Samuel Oschin Telescope at Palomar Observatory (P48), equipped with the 96 Mpixel mosaic camera CFH12K (Rahmer et al. 2008) and a Mould $R$ filter (Ofek et al. 2012). Additional $g$-band photometry of PTF12kso was obtained with the same telescope and instrument. PTF09gpn, PTF12gcx, and PTF12kso were also imaged in the Bgri bands with the automated Palomar 60-inch 

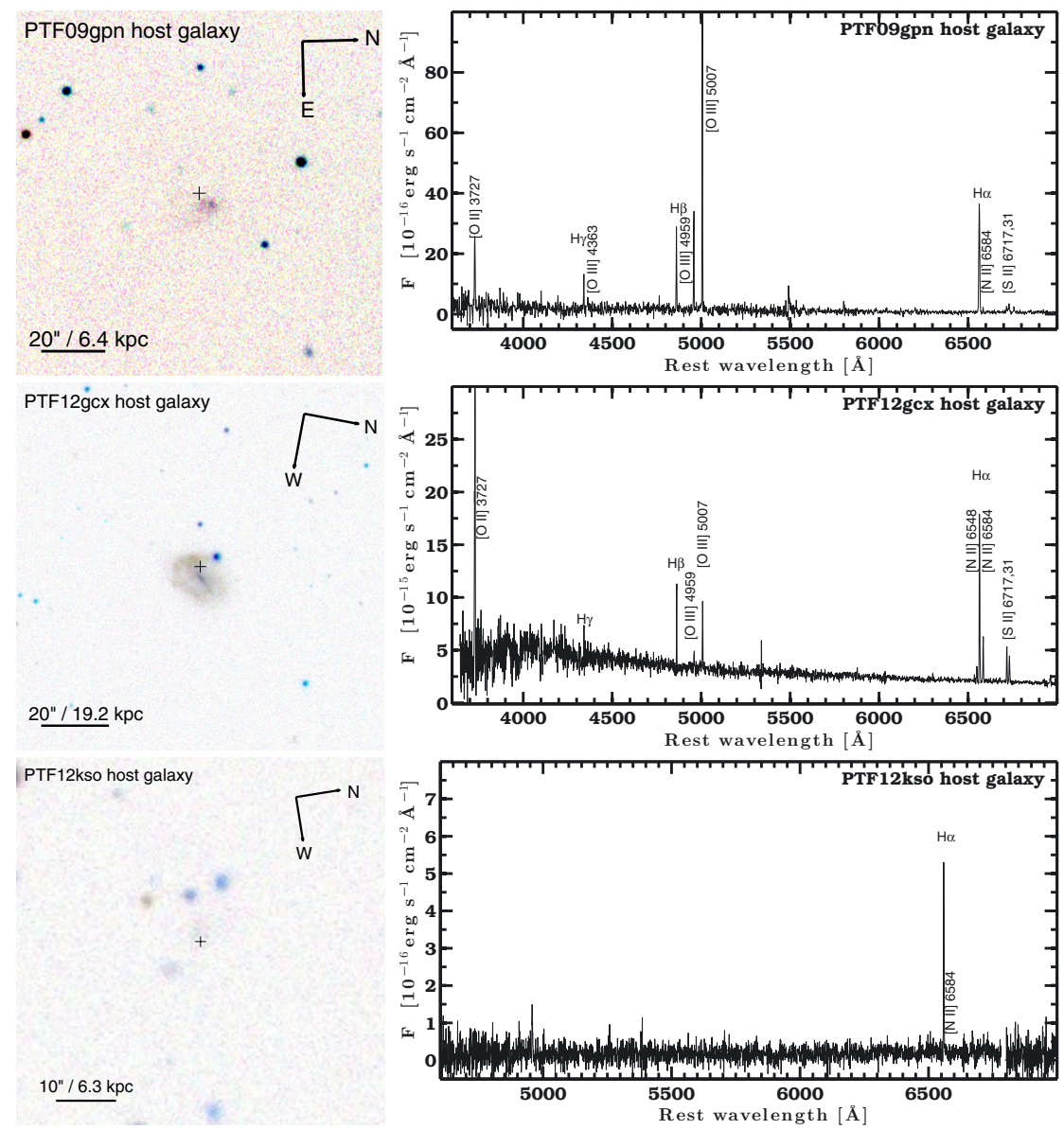

Fig. 3. Color images and spectra of the three host galaxies of our long-rising SNe II from PTF. The hosts of PTF09gpn and PTF12kso are particularly faint and metal poor. In the color images the position of the SN is marked by a black "+", and the scale as well as the orientation are reported. The color image of the host of PTF09gpn was obtained by combining three images in gri filters that we obtained with P60. The images of the other hosts are from SDSS. In the host-galaxy spectra, shown in the rest frame, we identified the most prominent emission lines. Some of them were used to estimate the metallicity.

telescope (P60; Cenko et al. 2006). PTF12gcx was not detected in the $B$ band, whereas for PTF09gpn $z$-band photometry was also obtained. For PTF12kso, a large number of photometric epochs with the 1-m telescopes of the Las Cumbres Observatory Global Telescope Network (LCOGT; Brown et al. 2013) in the griz bands were also obtained. For the same $\mathrm{SN}$ additional $B V R I$ photometry was obtained at Mount Laguna Observatory (MLO; Smith \& Nelson 1969) using the telescope, camera, and reduction technique described by Smith et al. (2015).

Point-spread-function (PSF) photometry was obtained on template-subtracted P48 and P60 images using the Palomar Transient Factory Image Differencing and Extraction (PTFIDE) pipeline $e^{2,3}$ and the $\mathrm{P} 60$ pipeline presented by Fremling et al. (in prep.). The photometry was calibrated against Sloan Digital Sky Survey (SDSS; Ahn et al. 2014) stars in the SN field (or observed the same night in other fields, in the case of PTF09gpn). The magnitudes are reported in Table A.2. For the LCOGT images of PTF12kso, we estimated the galaxy contribution by fitting a low-order surface and then performed PSF photometry.

A single optical spectrum of PTF09gpn was obtained using the P200+DBSP. Keck-1+LRIS was used to acquire an optical spectrum of the host galaxy. For PTF12gcx, three spectra were obtained with three different facilities: Keck 2+DEep Imaging Multi-Object Spectrograph (DEIMOS; Faber et al. 2003), P200+DBSP, and Lick 3-m+Kast. Four spectra of PTF12kso were obtained with P200+DBSP and Keck 2+DEIMOS, and

\footnotetext{
2 http://spider.ipac.caltech.edu/staff/fmasci/home/ miscscience/ptfide-v4.5.pdf

3 http://web.ipac.caltech.edu/staff/fmasci/home/ miscscience/forcedphot.pdf
}

one with the Copernico $1.82 \mathrm{~m}+$ AFOSC (obtained from the Asiago Supernova Classification program ${ }^{4}$ ). Spectra of the three host galaxies were obtained with P200+DBSP (PTF09gpn), from the SDSS archive (PTF12gcx), and with Keck 2+DEIMOS (PTF12kso). The spectra were reduced in a standard manner as outlined for the CCCP spectra. Table A.3 reports a log of the PTF spectral observations.

\section{Host galaxies}

We have already examined the host galaxies of the CCCP SN 1987A-like SNe in Taddia et al. (2013) along with those of the entire literature sample of long-rising SNe II. There we found that SN 1987A-like SN locations are more metal-poor than those of normal SNe II. These peculiar SNe are located either in the metal-poor outskirts of bright galaxies or in dwarf hosts. Here we present and analyze spectra and images of the PTF objects, which are shown in Fig. 3.

PTF09gpn was located in a faint host galaxy characterized by an apparent magnitude of 19.13 in the $r$ band (Arcavi et al. 2010). A color image of the galaxy is shown in Fig. 3 (top-left panel). The host-galaxy spectrum (top-right panel of Fig. 3) reveals several emission lines (Balmer lines, [O III] $\lambda \lambda 4363,4959$,

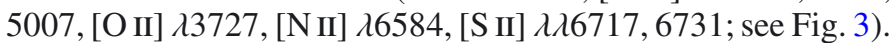

The absolute magnitude of the host galaxy in the $r$ band is $M_{r}=-15.35 \mathrm{mag}$, which is that of a dwarf galaxy. By using the value of the absolute magnitude in the $r$ band and Eq. (1) in Arcavi et al. (2010), we estimated the metallicity to be $0.13 Z_{\odot}$, or $12+\log (\mathrm{O} / \mathrm{H})=7.80\left(\right.$ where $12+\log (\mathrm{O} / \mathrm{H})_{\odot}=8.69 \mathrm{dex}$;

4 http://graspa.oapd.inaf.it/asiago_class.html 
Asplund et al. 2009). This would place PTF09gpn at the lowest end of the known metallicity distribution of SN 1987A-like events (Taddia et al. 2013).

To provide a more direct measurement of the host metallicity, we made use of the emission-line fluxes in the host-galaxy spectrum. We first modeled the spectrum to account for the Balmer absorption lines due to the underlying stellar population, using the STARLIGHT code (Cid Fernandes et al. 2005) ${ }^{5}$ as in Taddia et al. (2015a). Next we measured the Balmer-line fluxes and those of several other lines after subtracting the continuum; see Table A.4. We then computed the total extinction, which turned out to be negligible as measured from the Balmerline decrement (Osterbrock 1989). Different diagnostics, namely R23 (McGaugh 1991), N2, and O3N2 (Pettini \& Pagel 2004), were used to determine $12+\log (\mathrm{O} / \mathrm{H})$. R23 indicate an oxygen abundance of $12+\log (\mathrm{O} / \mathrm{H})_{\odot}=7.77 \pm 0.15$ (see Table A.4), confirming that PTF09gpn is characterized by one of the lowest metallicities measured so far. $\mathrm{N} 2$ and $\mathrm{O} 3 \mathrm{~N} 2$ indicate a somewhat higher metallicity $\left(12+\log (\mathrm{O} / \mathrm{H})_{\odot}=8.06 \pm 0.18 / 0.14\right)$, but they rely on the poor detection of [N II] $\lambda 6584(2.5 \sigma)$. Also, it is known that there are offsets between different strong-line diagnostics (Kewley \& Ellison 2008).

The bright $[\mathrm{O}$ III] emission makes the spectrum of this host similar to those of the so-called "green pea" (G.P.) galaxies (Cardamone et al. 2009) and H-poor SLSN hosts (Leloudas et al. 2015), which often show a preference for extreme emission-line galaxies (Amorín et al. 2015). The [O III] $\lambda 5007 /[\mathrm{O}$ II] $\lambda 3727$ ratio is 4.2, consistent with those of G.P. galaxies (Jaskot \& Oey 2013). The diameter of this galaxy is $\sim 10^{\prime \prime}$, which corresponds to $\sim 3.2 \mathrm{kpc}$, also consistent with the size of G.P. galaxies.

The $\mathrm{H} \alpha$ luminosity is $L(\mathrm{H} \alpha)=2.4 \times 10^{40} \mathrm{erg} \mathrm{s}^{-1}$. This gives an estimate of the star-formation rate (SFR) by applying the formula of Kennicutt \& Evans (2012): $\log _{10}(S F R)=$ $\log _{10} L(\mathrm{H} \alpha)-41.27$. We obtain $S F R=0.07 M_{\odot} \mathrm{yr}^{-1}$. The spectrum was calibrated against the $r$-band magnitude of the galaxy.

From the STARLIGHT fit we also obtained a value for the stellar mass $\left(M_{*}=1.24 \times 10^{8} M_{\odot}\right)$, which implies a specific star-formation rate (sSFR) of $5.6 \times 10^{-10} \mathrm{yr}^{-1}$.

PTF12gcx was hosted by a spiral galaxy, SDSS J154417.02+095743.8, characterized by SDSS apparent magnitudes of ugriz $=18.08(0.04), 16.92(0.01), 16.51(0.01)$, 16.25(0.01), 16.06(0.02) mag. A color image of the galaxy from SDSS is shown in Fig. 3 (center-left panel). The spectrum of its nucleus (see Fig. 3, center-right panel) reveals the same emission lines as shown by the host of PTF09gpn, with the exception of [O III] $\lambda 4363$ and $\mathrm{H} \delta$, which are too faint to be detected in this case.

The absolute magnitude of the host galaxy in the $r$ band is $M_{r}=20.08 \mathrm{mag}$, which is that of a bright spiral galaxy. Again, by using the value of the absolute magnitude in the $r$ band and Arcavi et al. (2010), we estimated a metallicity of $0.75 Z_{\odot}$, or $12+\log (\mathrm{O} / \mathrm{H})=8.56$. This places PTF12gcx at the highest end of the known metallicity distribution of SN 1987A-like events (Taddia et al. 2013), together with SNe 1998A, 2004em, and 2006 au.

We made use of the emission-line fluxes (see Table A.4) in the host-galaxy spectrum and strong-line diagnostics to further constrain the metallicity. The SN exploded relatively close to the host center (at 4 " separation), so the SDSS spectrum (obtained with a $3^{\prime \prime}$-wide fiber) can provide a good estimate of the metallicity at the position of PTF12gcx. N2 and O3N2 indicate $12+\log (\mathrm{O} / \mathrm{H})_{\odot}=8.52 \pm 0.18$ and $12+\log (\mathrm{O} / \mathrm{H})_{\odot}=8.56 \pm 0.14$

\footnotetext{
5 http://astro.ufsc.br/starlight/
}

(respectively), confirming that PTF12gcx is at relatively high metallicity. R23 indicates a slightly lower oxygen abundance of $12+\log (\mathrm{O} / \mathrm{H})_{\odot}=8.27 \pm 0.15($ see Table A.4).

The $\mathrm{H} \alpha$ luminosity is $L(\mathrm{H} \alpha)=2.5 \times 10^{40} \mathrm{erg} \mathrm{s}^{-1}$, which corresponds to a SFR of $0.13 M_{\odot} \mathrm{yr}^{-1}$ (Kennicutt \& Evans 2012). The spectrum was calibrated against the SDSS $r$-band magnitude of the galaxy. With $M^{*}=6.4 \times 10^{9} M_{\odot}$, we obtain $s S F R=2.0 \times 10^{-11} \mathrm{yr}^{-1}$.

PTF12kso was hosted by an extremely faint galaxy, barely detected in the SDSS images (see Fig. 3, bottom-left panel). Aperture photometry on stacked P48 $r$-band images reveals a host apparent magnitude of $r=20.64 \mathrm{mag}$. The absolute magnitude of -15.12 mag implies a metallicity of $Z=0.0024=$ $0.12 Z_{\odot}$, or $12+\log (\mathrm{O} / \mathrm{H})=7.76$, which makes the host of PTF12kso one of the most metal-poor SN 1987A-like SN hosts observed so far.

The spectrum of this faint host galaxy exhibits strong $\mathrm{H} \alpha$ emission and a faint continuum. We use the flux of $\mathrm{H} \alpha$ and the limit on the flux of [N II] to estimate an oxygen abundance of $12+\log (\mathrm{O} / \mathrm{H}) \lesssim 8.04$ with the $\mathrm{N} 2$ method. The SFR obtained from the $\mathrm{H} \alpha$ luminosity is $S F R=0.015 M_{\odot} \mathrm{yr}^{-1}$. The spectral continuum is too faint and noisy to obtain a reliable $M^{*}$ estimate from the STARLIGHT fit.

In summary, two out of our three new metallicity measurements push the oxygen-abundance distribution of the host galaxies of SN 1987A-like SNe shown in Taddia et al. (2013) to even lower values, making the SN 1987A-like SNe one of the most metal-poor populations of CC SNe, together with SN impostors (Taddia et al. 2015b) and H-poor SLSN hosts (Leloudas et al. 2015).

\section{Supernova light curves and colors}

\subsection{Light-curve shape}

\subsubsection{PTF supernovae}

PTF09gpn was imaged in the Bgriz bands, and the light curves (with the exception of the late $r$-band epochs) are reported in Fig. 4 (left-hand panel). The $r$-band light curve of PTF09gpn covers the first $\sim 350 \mathrm{~d}$. The $r$-band light curve sampling is rather frequent ( $2.4 \mathrm{~d}$ average cadence) until $\sim 72 \mathrm{~d}$. Several epochs were taken at late times ( 300 to $350 \mathrm{~d}$ ). In the other bands the follow-up observations started $\sim 2$ weeks after discovery, with an average cadence of $4.1,3.6,5.7$, and $5.7 \mathrm{~d}$ for $z, i, g$, and $B$ (respectively). In these filters the last photometric epoch was taken at $\sim 82 \mathrm{~d}($ Bgi $)$ and $\sim 72 \mathrm{~d}(z)$. The $r$-band light curve shows a rise of $\sim 0.8 \mathrm{mag}$ in the first $10 \mathrm{~d}$. Then it settles onto an early plateau between days 10 and 30, and it finally starts a long rise to peak ( $\sim 0.9$ mag brighter than the plateau) that occurs at $\sim 75 \mathrm{~d}$. All of the other bands reach maximum around the same time, and between days 10 and 30 they also exhibit an early-time plateau, preceded by a decline in the case of $B$ and $g$. Since the followup observations started 2 weeks later for these filters than in the $r$ band, no early-time rise could be detected. The overplotted and scaled light curves of SN 2006au (from Taddia et al. 2012) clearly show the close resemblance of PTF09gpn to this SN 1987A-like SN.

PTF12gcx was imaged in the $r$ band with P48. The light curve (central panel of Fig. 4) is characterized by frequent sampling, with the SN being imaged every 1.3 nights on average during the rise $(\sim 1.4 \mathrm{mag}$ from discovery to peak). Three epochs (the last at $\sim 100 \mathrm{~d}$ ) were taken after peak, which occurred at day 57. After peak the decline is of $\sim 1 \mathrm{mag}$ in $\sim 40 \mathrm{~d}$. The light-curve shape is very similar to that of SN 1987A, which is also shown in the figure. A difference is that PTF12gcx rises 

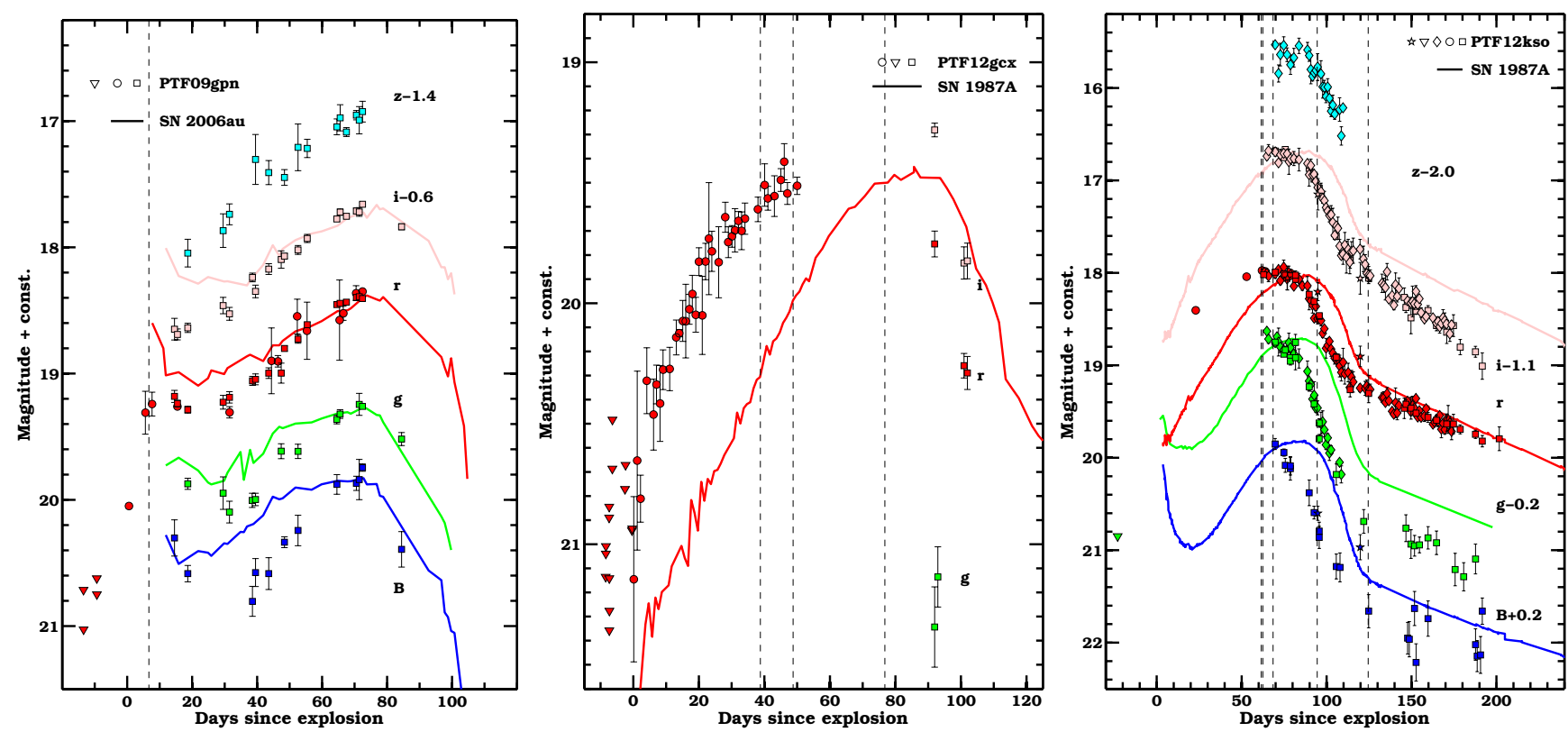

Fig. 4. Optical light curves of PTF09gpn, PTF12gcx, and PTF12kso. The circles show detections obtained with the P48 telescope and the squares indicate observations with the P60 telescope in Bgriz. Diamonds and stars mark the LCOGT and MLO photometry (obtained on the JohnsonCousins BVRI photometric system; see Table A.2) for PTF12kso. Triangles indicate prediscovery $3 \sigma$ limiting magnitudes from P48 observations. The corresponding light curves of SN 2006au (from Taddia et al. 2012) and SN 1987A (solid lines) are overplotted and shifted in magnitude to match those of our objects. The vertical dashed lines mark the spectroscopic epochs.

faster in the first days after discovery and thus peaks earlier than SN 1987A. A few epochs with P60 in gri were also taken after the peak.

The light curves of PTF12kso are shown in the right-hand panel of Fig. 4. This SN was imaged in the Bgriz bands. The first observation in $r$ with P48 occurred at $\sim 20 \mathrm{~d}$. Then, after a gap of $\sim 20 \mathrm{~d}$ in the light-curve coverage, at $\sim 40 \mathrm{~d}$ intense follow-up observations started in all the bands with P60 and in griz with LCOGT. The SN was observed and detected by LCOGT almost every night from a few days before peak (which occurred at $61 \mathrm{~d}$ in $r$ ) until $\sim 170 \mathrm{~d}(\sim 110 \mathrm{~d}$ in $g$ and $z)$. P60 took additional images up to $200 \mathrm{~d}$. After peak the light curves decline by $\sim 1 \mathrm{mag}$ in $\sim 60 \mathrm{~d}$, but then after $120 \mathrm{~d}$ the decline rate becomes smaller by about $50 \%$, with the light curve setting onto a tail very similar to that observed in SN 1987A.

Rise times and peak magnitudes for the PTF SNe are summarized in Table 3.

\subsubsection{CCCP supernovae}

SN 2004ek was observed in BVRI by Arcavi et al. (2012) and Tsvetkov (2008). The light curves are shown in Fig. 5 (top-left panel). The monitoring started at $\sim 13 \mathrm{~d}$ and, before maximum (which occurred at $\sim 90 \mathrm{~d}$ in $V R I$ ), the light-curve sampling was rather frequent, with the SN observed every 2.7 nights on average. After peak, a few epochs were obtained between $\sim 130$ and $\sim 160$ d. The last observations occurred at almost $290 \mathrm{~d}$ in all bands except $B$, for which the last epoch was obtained at $\sim 130 \mathrm{~d}$. The light curves of SN 2004ek show an early decline up to $\sim 25-$ $32 \mathrm{~d}$ in all the bands except $I$, which shows an early plateau of comparable length. The decline is faster in the bluer bands, with $B$ and $R$ exhibiting a decline of $\sim 0.5$ and 0.2 mag from the early peak, respectively. Following this phase, the VRI bands show a slow rise to peak $(\sim 0.4 \mathrm{mag}$ in $\sim 55$ days), which occurred at $\sim 80$ days. At the same epochs, the $B$ band shows a plateau. The early, long-lasting declining phase and the slow rise to maximum shown by SN 2004ek make this SN a very peculiar object - sort of an intermediate case between SN 1987A and a normal SN IIP such as SN 1999em, as we show in Fig. 5. The post-maximum decline up to $160 \mathrm{~d}$ is also quite slow in all of the redder bands, as compared with SNe 1987A and 1999em. However, the last epoch obtained at $290 \mathrm{~d}$ shows a difference in magnitude from peak comparable to that of SN 1999em and larger than that of SN 1987A.

SN 2004em has BVRI light-curve coverage and shape that are rather similar to those of SN 2004ek (see Fig. 5, top-right panel). For this SN the monitoring began at $\sim 2$ weeks, and most of the epochs were obtained before maximum (which occurred at $\sim 110$ d). Before maximum the $\mathrm{SN}$ was observed once per week on average. A few additional epochs were obtained at $\sim 140 \mathrm{~d}$, and the last one at $275 \mathrm{~d}$. This SN shows a $0.6 \mathrm{mag}$ decline in the $B$-band light curve in the first $\sim 40$ days. At the same epochs, the $V$ and $R$ bands appear constant, whereas the $I$ band first rises by 0.5 mag for $\sim 2$ weeks, and then also shows a short plateau up to $\sim 40$ days. After the early phase, all of the light curves rise to peak. The rise to peak is steeper than in the case of SN 2004ek, reaching about $1 \mathrm{mag}$ over $50 \mathrm{~d}$ in $R$. After peak, the decline is slower than for SN 1987A, as shown in the figure. The last epoch shows that the difference in magnitude from peak to tail is similar to that of SN 1987A.

The light curves of SN 2005ci are rather similar to those of SN 1987A, as shown in Fig. 5 (bottom-left panel). This SN was observed by Arcavi et al. (2012) in BVRI and unfiltered $(\sim R)$ by Kleiser et al. (2011). The CCCP follow-up observations started at $11 \mathrm{~d}$, with an average cadence of 4 nights and the last epoch taken at $130 \mathrm{~d}$. The unfiltered light curve has the first epoch only a few hours after explosion, and covers the first $44 \mathrm{~d}$. The rise to peak, which occurs at $\sim 87 \mathrm{~d}$ in $R$, is faster than for SN 1987A in the first $\sim 30-40$ days in all bands. Thereafter, the slope becomes less steep $(\sim 0.5 \mathrm{mag}$ in $\sim 50 \mathrm{~d}$ in $R)$. The $B$ band shows hints of an early plateau before day 20 . Its shape is flatter than that of SN 1987A. The post-peak decline $(\sim 1 \mathrm{mag}$ in $\sim 40 \mathrm{~d}$ in the $R$ band) is similar to that of SN 1987A. 

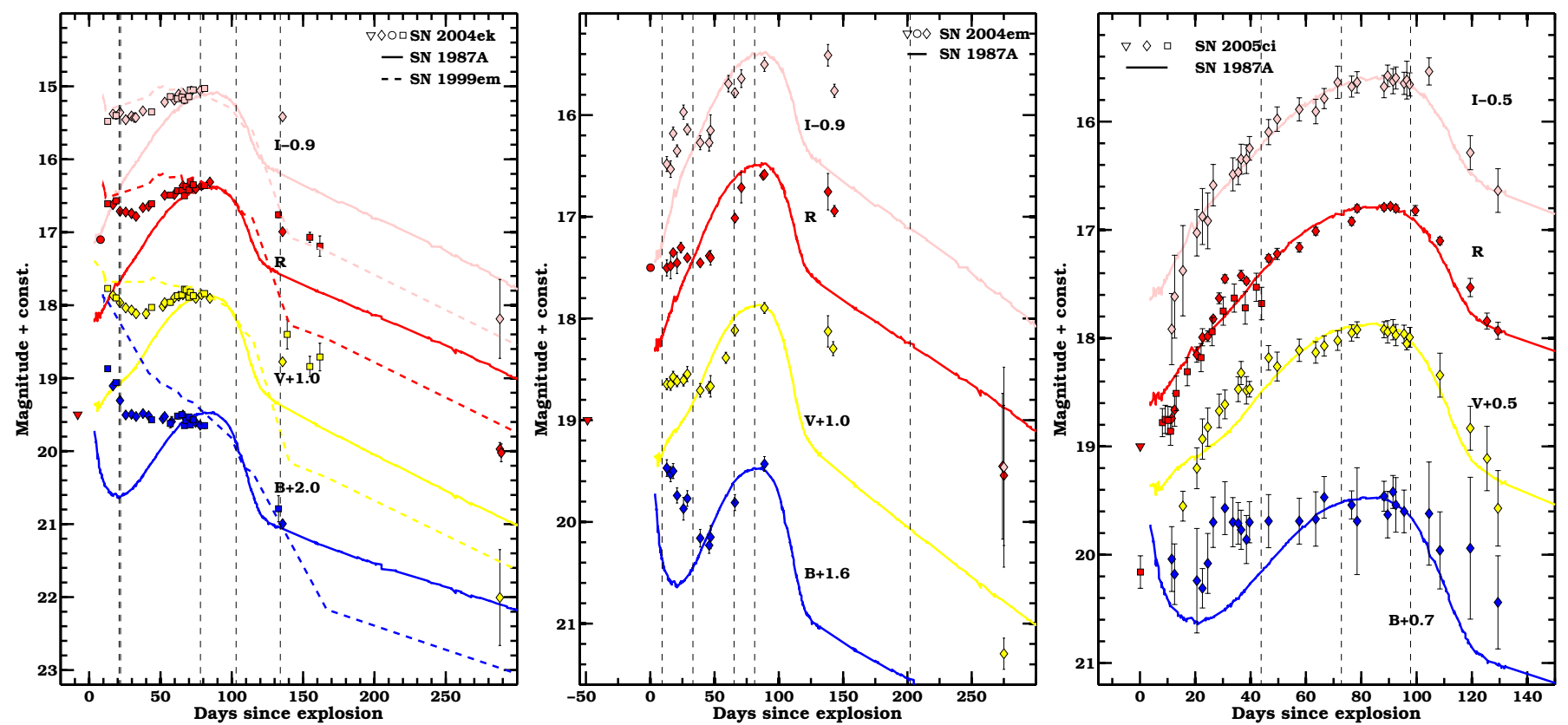

Fig. 5. Optical light curves of SNe 2004ek, 2004em, and 2005ci. Diamonds indicate CCCP photometry, squares indicate photometry from Tsvetkov (2008) for SN 2004ek and from Kleiser et al. (2011) for SN 2005ci. For SNe 2004ek and 2004em, the circle indicates the discovery magnitude from Monard et al. (2004) and Armstrong (2004). The BVRI photometry from Tsvetkov (2008) was shifted by $+0.1,+0.2,+0.2$, and +0.1 mag (respectively), in order to match the CCCP photometry of SN 2004ek. Triangles indicate limits from prediscovery nondetections from the literature (see Table 2). The corresponding light curves of SNe 1987A (solid lines) and 1999em (from Elmhamdi et al. 2003, dashed lines) are overplotted and shifted in magnitude to match the peaks of our objects. The vertical dashed lines mark the spectroscopic epochs.

Table 3. Rise times and peak magnitudes of long-rising SNe II.

\begin{tabular}{l|cc|cc|cc}
\hline \hline & $\begin{array}{c}t_{\text {rise }} \\
(\text { days })\end{array}$ & $\begin{array}{c}m_{\max } \\
(\mathrm{mag})\end{array}$ & $\begin{array}{c}t_{\text {rise }} \\
(\text { days })\end{array}$ & $\begin{array}{c}m_{\max } \\
(\mathrm{mag})\end{array}$ & $\begin{array}{c}t_{\text {rise }} \\
(\text { days })\end{array}$ & $\begin{array}{c}m_{\max } \\
(\mathrm{mag})\end{array}$ \\
\hline Band/SN & SN 2004ek & & SN 2004em & & SN 2005ci & \\
$B$ & 55 & 17.55 & $\ldots$ & $\ldots$ & 74 & 18.85 \\
$V$ & 75 & 16.84 & 111 & 16.61 & 86 & 17.43 \\
$R$ & 83 & 16.35 & 108 & 16.47 & 87 & 16.77 \\
$I$ & 94 & 15.90 & 115 & 15.93 & 89 & 16.09 \\
\hline Band/SN & PTF09gpn & & PTF12gcx & & PTF12kso & \\
$B$ & 77 & 19.74 & $\ldots$ & $\ldots$ & $\ldots$ & $\ldots$ \\
$g$ & 77 & 19.26 & $\ldots$ & $\ldots$ & $\ldots$ & $\ldots$ \\
$r$ & $>72$ & $<18.35$ & 68 & 19.32 & 68 & 18.00 \\
$i$ & 77 & 18.26 & $\ldots$ & $\ldots$ & 68 & 17.78 \\
$z$ & $>72$ & $<18.32$ & $\ldots$ & $\ldots$ & 79 & 17.60 \\
\hline
\end{tabular}

Notes. Rise times and peak magnitudes are obtained from low-order polynomial fits to the SN light curves.

Rise times and peak magnitudes for the CCCP SNe are summarized in Table 3.

\subsection{Absolute magnitudes}

In Fig. 6 we plot the absolute magnitudes in $B, g, V, r / R / u n f$., and $i / I$ filters of all our $\mathrm{SNe}$ (colored symbols) and of other well-observed long-rising SNe II resembling SN 1987A (black symbols and lines). The literature data were taken from Kleiser et al. (2011), Pastorello et al. (2005), Pastorello et al. (2012), Taddia et al. (2012), and reference therein. For our SNe we adopted the distances and extinctions listed in Table 1. For the $\mathrm{SNe}$ in the literature we obtained redshift-independent distances from NED when available; otherwise we adopted WMAP5 cosmological parameters and a redshift from NED, to be consistent with the distances computed for our objects. The Galactic extinctions were obtained from NED. The explosion epochs and the host-galaxy extinctions were obtained from the literature.
The only band in which all our objects and those from the literature have a frequently sampled and extended light curve is $r / R /$ unf. The comparison in this band reveals that the magnitude of the (second) peak ranges from $-16.0 \mathrm{mag}$ (as in the case of SNe 2005ci, 2009E, and PTF09gpn) to -18.5 mag (SN 2004ek). One PTF SN (PTF12kso) and two CCCP objects (SNe 2004ek and 2004em) are more luminous than all of the other SN 1987Alike events previously published (among those SNe, SN 2006V was hitherto the most luminous), peaking at $M_{r / R}<-17.3$ mag. PTF09gpn and SN 2005ci are instead among the least luminous SN 1987A-like SNe, being only marginally brighter than SN 2009E. On the tail (i.e., after $\sim 130$ d), the SNe which have brighter peaks also have brighter tails. However, at very late epochs ( $290 \mathrm{~d})$, the absolute magnitudes of SNe 2004ek and $2004 \mathrm{em}$ are slightly brighter (up to $0.4 \mathrm{mag}$ ) than those of SN 1998A, which at peak was up to 1.5 mag fainter. Compared to the $r / R$ band, the $B$ and $V$ bands exhibit wider ranges of peak magnitudes (3.5 and 2.5 mag, respectively). 
F. Taddia et al.: PTF and CCCP long-rising SNe II

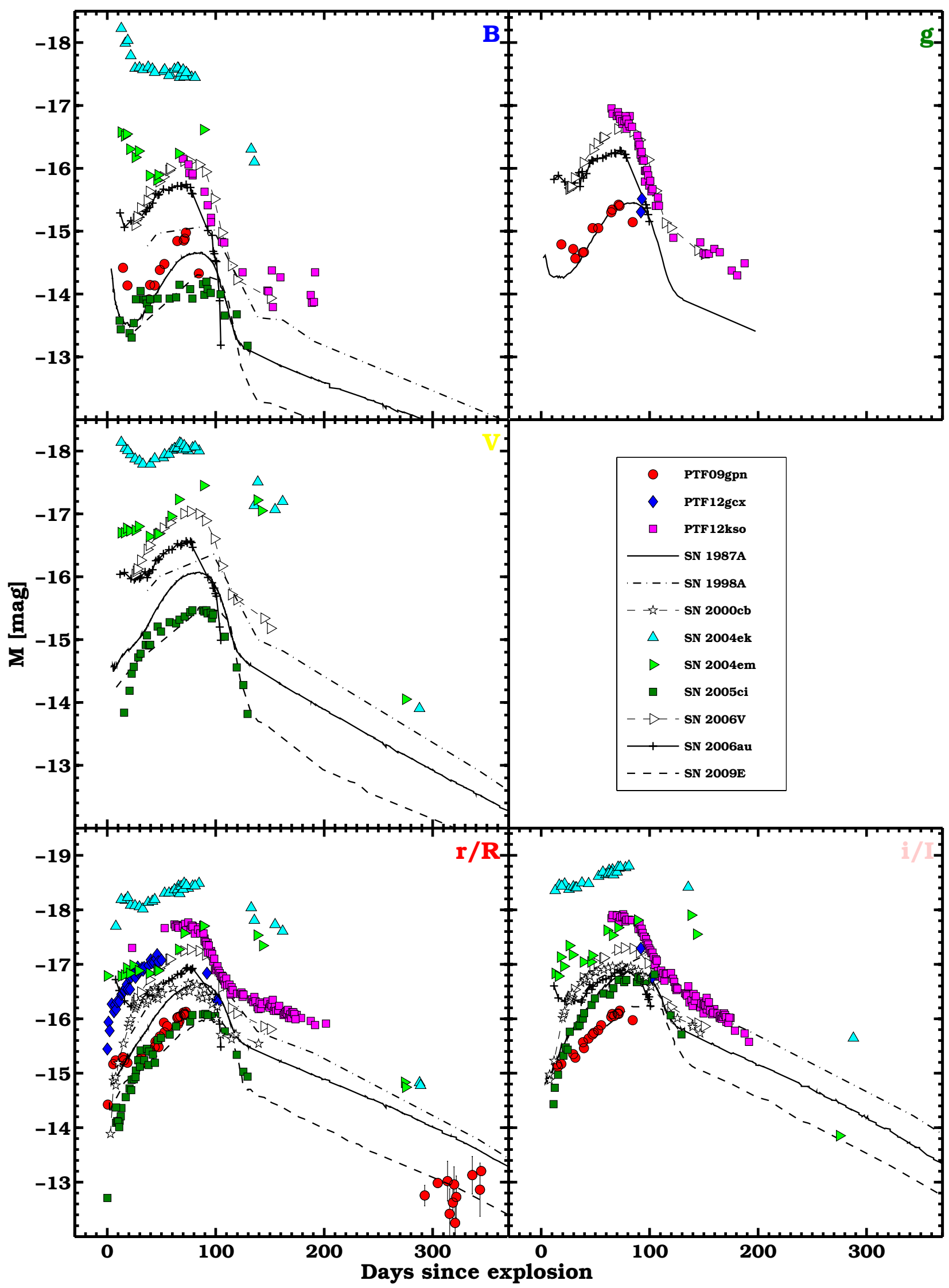

Fig. 6. $B, g, V, r / R$, and $i / I$-band absolute magnitudes for our PTF and CCCP SN 1987A-like SNe (colored symbols) and for similar events (black lines and symbols) from the literature (Kleiser et al. 2011; Pastorello et al. 2005, 2012; Taddia et al. 2012; and references therein). 


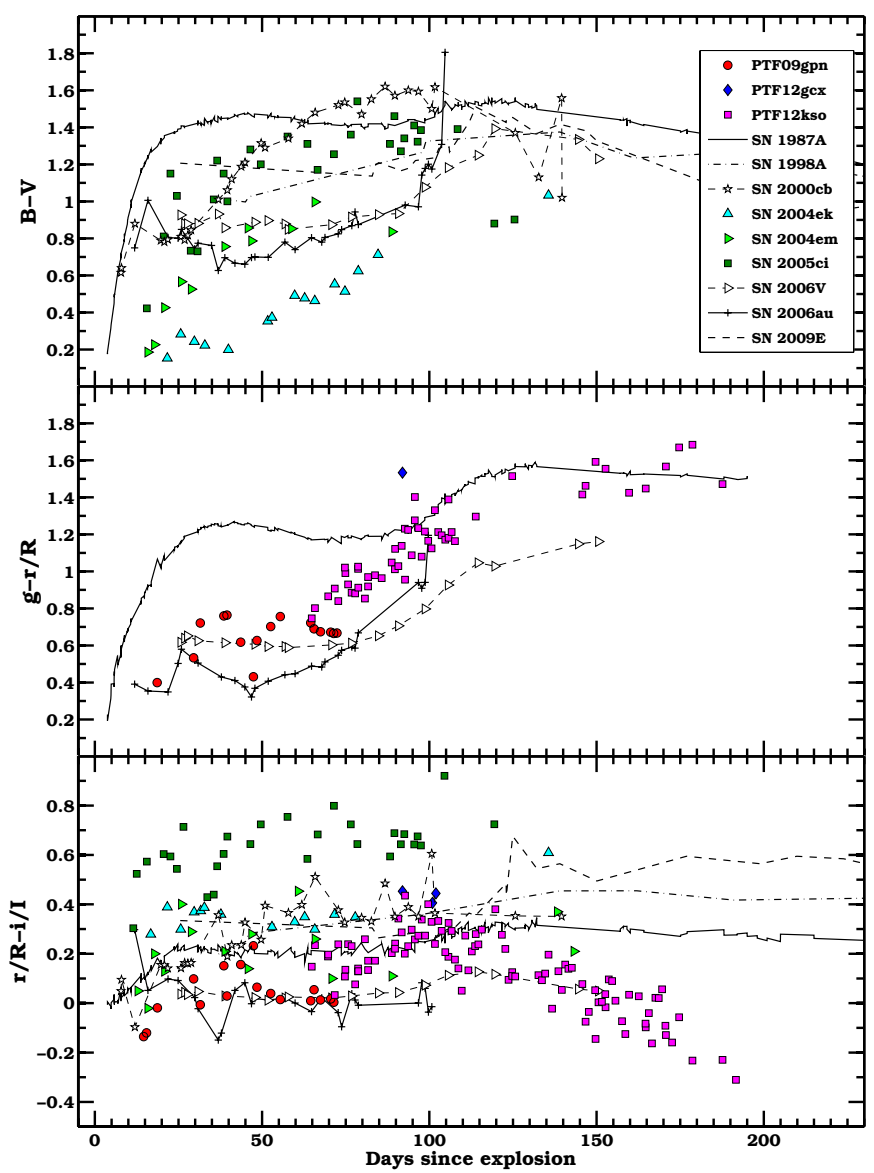

Fig. 7. Color comparison among our PTF and CCCP SN 1987A-like $\mathrm{SNe}$ (colored symbols) and similar events (black lines and symbols) from the literature (Kleiser et al. 2011; Pastorello et al. 2005, 2012; Taddia et al. 2012; and references therein).

At early phases, when most of the flux is in the bluer bands, $B$ ranges from $-18.2 \mathrm{mag}$ (SN 2004ek) down to $-13.3 \mathrm{mag}$ (SN 2005ci). In between, we find SNe 1987A, 2004em, 2006au, and PTF09gpn, showing early declines.

The large range of absolute magnitudes has important implications for the progenitor parameters, in particular for the ${ }^{56} \mathrm{Ni}$ mass and the progenitor radius (see Sect. 8).

\subsection{Colors}

Adopting the light curves and the extinctions used to compute the absolute magnitudes, we also compared the colors of our $\mathrm{SNe}$ to those of other long-rising SNe. In Fig. 7 we plot three different optical colors: $B-V, g-r / R$, and $r / R-i / I$. The $B-V$ and $g-r / R$ color evolutions are similar for all of the $\mathrm{SNe}$, with bluer colors at early epochs which progressively become redder. Among the reddest SNe we find SN 2005ci, similar to SN 1987A. SNe 2004ek, 2004em, PTF09gpn, and PTF12kso are bluer, similar to SNe 2006V and 2006au.

The $r / R-i / I$ color looks flatter for all of the $\mathrm{SNe}$, as its value is more influenced by the different strengths of the spectral lines (e.g., $\mathrm{H} \alpha$ and $\mathrm{Ca}$ II) than by the continuum.

\section{Supernova spectra}

\subsection{PTF SN spectra}

In Fig. 8 we plot the spectral sequences of PTF09gpn, PTF12gcx, and PTF12kso. The spectra are made publicly available via WISeREP (Yaron \& Gal-Yam 2012). All of the spectra were calibrated against $r$-band photometry and corrected for reddening.

In Fig. 8 (top panel) we show the only spectrum obtained of PTF09gpn, which was taken at $\sim 7 \mathrm{~d}$, during the early peak of the $r$-band light curve, as indicated by the vertical dashed line in Fig. 4 (left-hand panel). The Balmer lines ( $\mathrm{H} \alpha, \mathrm{H} \beta, \mathrm{H} \gamma, \mathrm{H} \delta, \mathrm{H} \epsilon)$ dominate the spectrum of PTF09gpn and are labeled in the figure. All of them exhibit a broad P-Cygni profile. The continuum appears quite blue, as the spectrum was obtained at early times.

In the central panel of Fig. 8 three spectra of PTF12gcx are reported. The first two spectra, obtained on the rise of the light curve at 39 and $49 \mathrm{~d}$, show clear P-Cygni profiles for the Balmer lines (from $\mathrm{H} \alpha$ to $\mathrm{H} \delta$ ), for $\mathrm{Na}$ I D, and for the Ca II triplet. Redward of Na I D there may be traces of high-velocity $\mathrm{H} \alpha$ absorption or Si II $\lambda 6355$. There are also signatures of Fe II $\lambda 5169$ in absorption. The continuum spectral energy distribution (SED) peaks in the $V$ band. The last spectrum, obtained after peak, has low signal-to-noise ratio and only reveals a faint $\mathrm{H} \alpha$ feature.

The spectral sequence of PTF12kso is shown in the bottom panel of Fig. 8. Five spectra were obtained, three of them before peak (at 61 to $68 \mathrm{~d}$ ) and two after peak (at 94 and $124 \mathrm{~d}$ ). In the early-time spectra, besides the Balmer lines down to $\mathrm{H} \epsilon$, we detect P-Cygni features associated with $\mathrm{Na} \mathrm{I} \mathrm{D} \mathrm{(possibly} \mathrm{blended}$ with $\mathrm{He}$ I 15875), Ca II triplet, Fe II triplet, and probably Ca II H\&K. There are also traces of Ba II at $6142 \AA$ and $4554 \AA$. $\mathrm{Ba}$ II $\lambda 6142$ may be blended with high-velocity $\mathrm{H} \alpha$ absorption or Si II $\lambda 6355$. The continuum SED peaks in the $V$ band. In the last spectrum, the absorption part of the P-Cygni features decreases its strength in all the lines except for $\mathrm{Na}$ I D. At these late epochs, Ca II $\lambda \lambda 7291,7323$ emerges in emission, the continuum is faint and almost constant, and the lines dominate the SED.

\subsection{CCCP SN spectra}

In Fig. 9 we plot the spectral sequences of $\mathrm{SNe} 2004 \mathrm{ek}$, 2004em, and 2005ci. The spectra are made publicly available via WISeREP (Yaron \& Gal-Yam 2012). All the spectra were calibrated against $R$-band photometry and corrected for reddening.

The spectra of SN 2004ek were obtained at 21, 22, 78 (prepeak), 103 and 134 (post-peak) days. The first two spectra were taken at the epoch of the early cooling phase, and they show a blue continuum where diluted P-Cygni profiles of Balmer lines are barely visible. Fe II $\lambda 5169$ and possibly He I $\lambda 5875$ in absorption are also visible. The later spectra peak in the continuum around 5000-5500 $\AA$, and show strong broad $\mathrm{H} \alpha$ emission with a marginal and narrow P-Cygni absorption. The other Balmer lines and in particular $\mathrm{H} \beta$ show prominent P-Cygni features. Na I D emerges and Fe II $\lambda 5169$ (along with Fe II $\lambda 5018$ ) becomes more evident. The Ca II triplet emerges and traces of Ba II $\lambda \lambda 4554$, 6142 are observable. Possibly Ca II H\&K is also detected.

The first spectrum of SN 2004em was obtained at $8 \mathrm{~d}$, at the cooling phase. Because of the early phase, it appears much bluer than the other spectra, and shows broad Balmer P-Cygni profiles. The next three spectra were also obtained before maximum, at 33, 65, and $81 \mathrm{~d}$. Although the second spectrum was taken on the decline of the $B$-band light curve, it does not appear as blue as the first two spectra of SN 2004ek or as the previous spectrum of SN 2004em. These spectra have a continuum that peaks in the $V$ band, and are dominated by the typical SN II PCygni Balmer profiles. As in the spectra of the SNe that were previously discussed, they exhibit broad P-Cygni profiles for $\mathrm{Na} I \mathrm{D}$ and for the $\mathrm{Ca}$ II and Fe II triplets. In the last spectrum, 


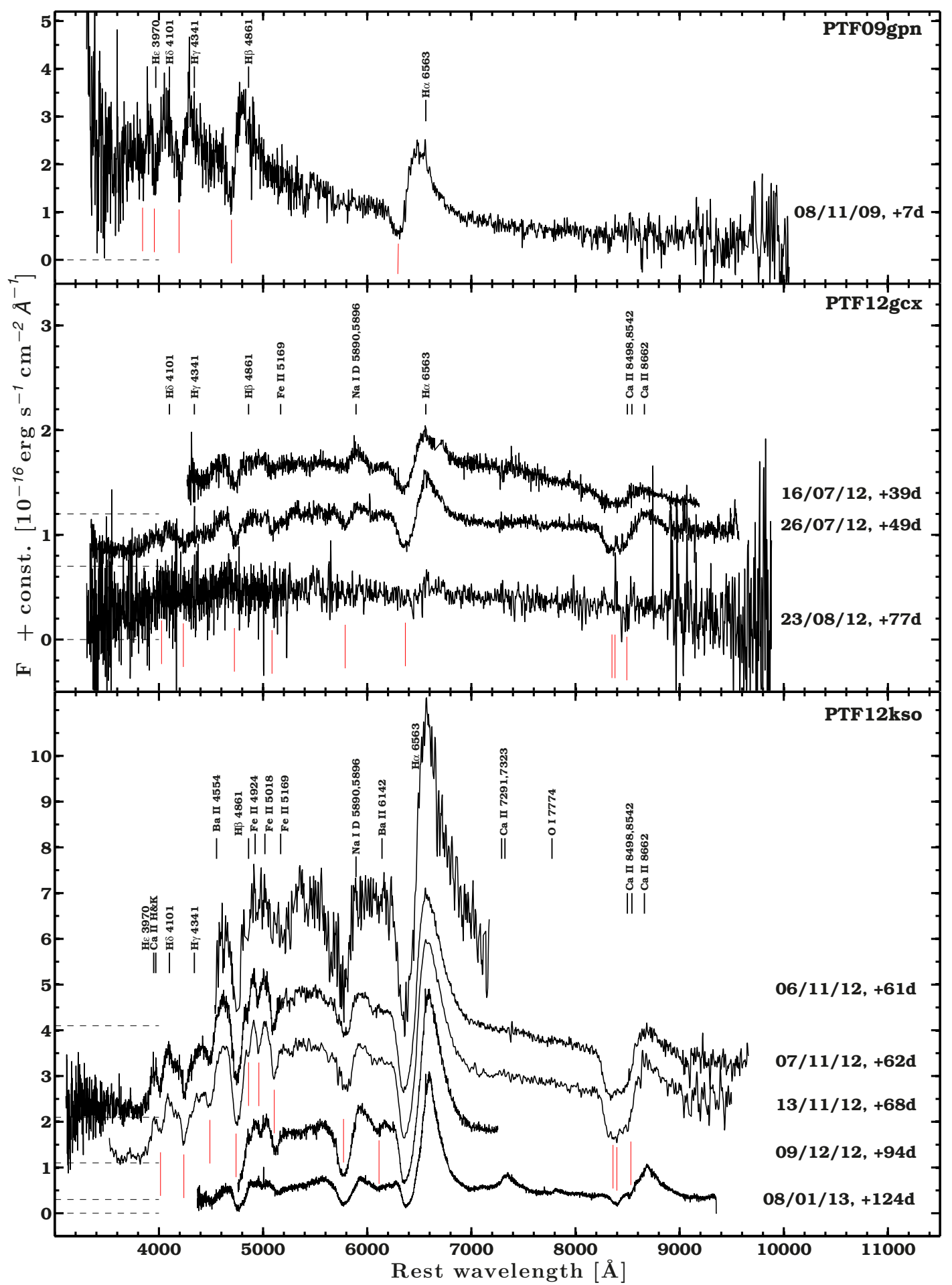

Fig. 8. Spectral sequences for PTF09gpn, PTF12gcx, and PTF12kso. All spectra were calibrated against photometry and corrected for extinction. The main spectral lines, the dates, and the phases are reported. The absorption minimum of each line is marked by a red segment, the rest wavelength by a black segment. The zero-flux level is marked by a dashed horizontal line for each spectrum.

taken at $202 \mathrm{~d}$, Ca II $\lambda \lambda 7291,7323$ emerges in emission, the Ca II triplet does not show absorption, the continuum becomes weak, and $\mathrm{H} \alpha$ dominates the SED with a strong broad emission and residual absorption.

SN 2005ci has three spectra obtained at 44, 72, and $98 \mathrm{~d}$ (only one after the peak and before the tail). These spectra are particularly rich in features and are dominated by P-Cygni Balmer lines. All of the other lines mentioned for the other $\mathrm{SNe}$ are visible in emission and absorption, including $\mathrm{Na}$ I D,
Ca II triplet, Fe II $\lambda \lambda 4924,5018,5169$, Ca II $\lambda \lambda 7291,7323$ (in emission), and Ba II $\lambda \lambda 4554,6142$. The spectral continua are quite red, peaking around $6000 \AA$.

\subsection{Spectral comparison and expansion velocities}

For a more direct spectral comparison among our SNe and other SN 1987A-like events, we plot a sequence of spectra taken at 


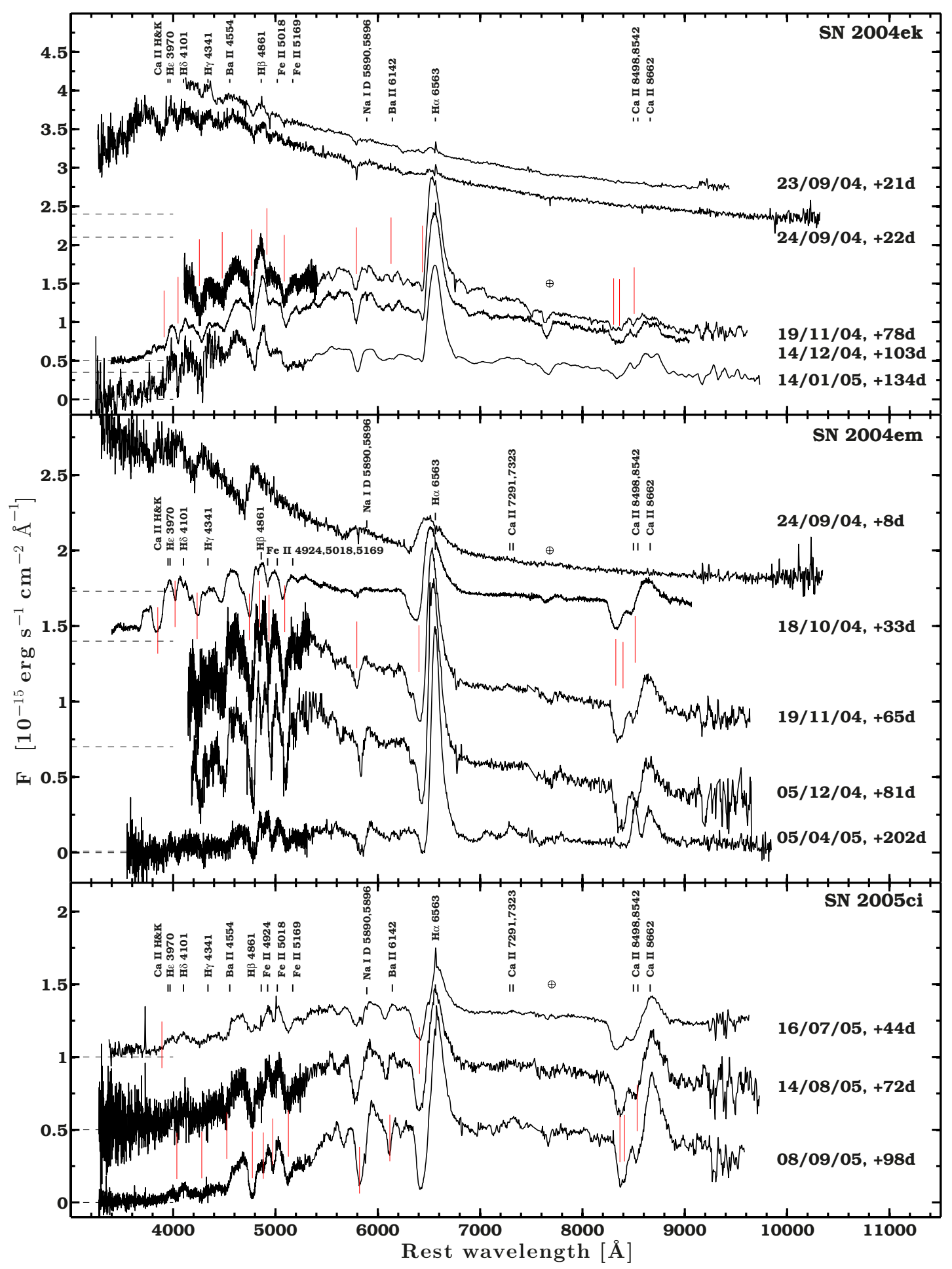

Fig. 9. Spectral sequences for SNe 2004ek, 2004em, and 2005ci. All spectra were calibrated against photometry and corrected for extinction. The main spectral lines, the dates, and the phases are reported. The absorption minimum of each line is marked by a red segment, the rest wavelength by a black segment. The zero-flux level is marked by a dashed horizontal line for each spectrum. Telluric features are marked by $\oplus$.

early epochs in the top panel of Fig. 10, one taken around peak in the central panel, and three spectra obtained at late epochs in the bottom panel.

In the top panel it is evident that at early epochs these longrising SNe II show Balmer and (later) Fe features diluted by a strong black-body continuum. The case of SN 2004ek is particularly extreme, with the spectral lines almost completely diluted even after 3 weeks from the explosion. As the ejecta expand, the continuum becomes less prominent and more features emerge.
These lines characterize the spectra taken around peak, shown in the central panel. Here we can see the SNe exhibit different line broadening, corresponding to different expansion velocities.

The late-time spectra shown in the bottom panel highlight the transition to the nebular phase, with a faint continuum and strong emission lines, in particular $\mathrm{H} \alpha$ and $\mathrm{Ca}$ II.

We measured the expansion velocities of our SNe from the P-Cygni minima of different lines at all available epochs, and 


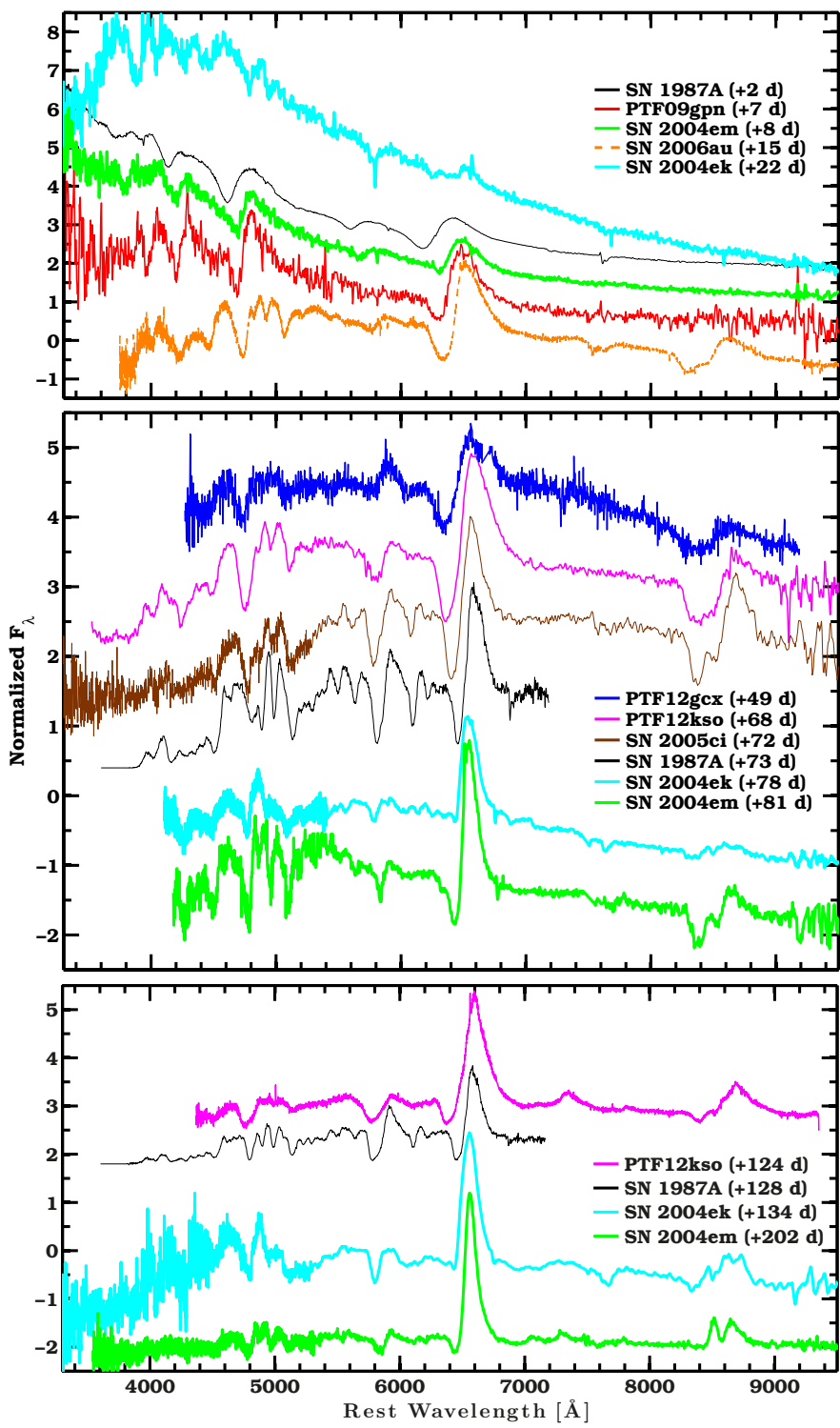

Fig. 10. Spectral comparison among our PTF and CCCP long-rising SNe II, SN 1987A (from Phillips et al. 1988) and SN 2006au (from Taddia et al. 2012). The top panel includes spectra taken at early epochs, in the central panel we show spectra obtained around peak. The bottom panel presents spectra taken on the tail of the light curves.

display the results in Fig. 11 . Balmer lines, Fe II $\lambda 5169$, Na I D, Ba II $\lambda 6142$, and Ca II $\lambda 8662$ are analyzed.

Starting with the Fe II $\lambda 5169$ line velocities, which better represent the photospheric velocities (Dessart \& Hillier 2005), we can see how our SNe are faster than SN 1987A and SN 2009E, and similar to the more energetic SNe 1998A, 2006V and 2006au. In particular, SNe 2004ek and 2004em exhibit the fastest photospheres. SN 2005ci is the slowest in our sample. These results are confirmed when we look at the Ba II lines, another good indicator of the photospheric velocity.

Looking at the bright $\mathrm{H} \alpha$ line, we observe that PTF12kso and PTF12gcx have larger velocities compared to the other SNe, which show similar expansion velocities as did SN 1987A. This is also observed in $\mathrm{H} \beta$, but the difference is less significant.

$\mathrm{Na}$ I D and especially the Ca II lines show similar velocities in all of the SNe.

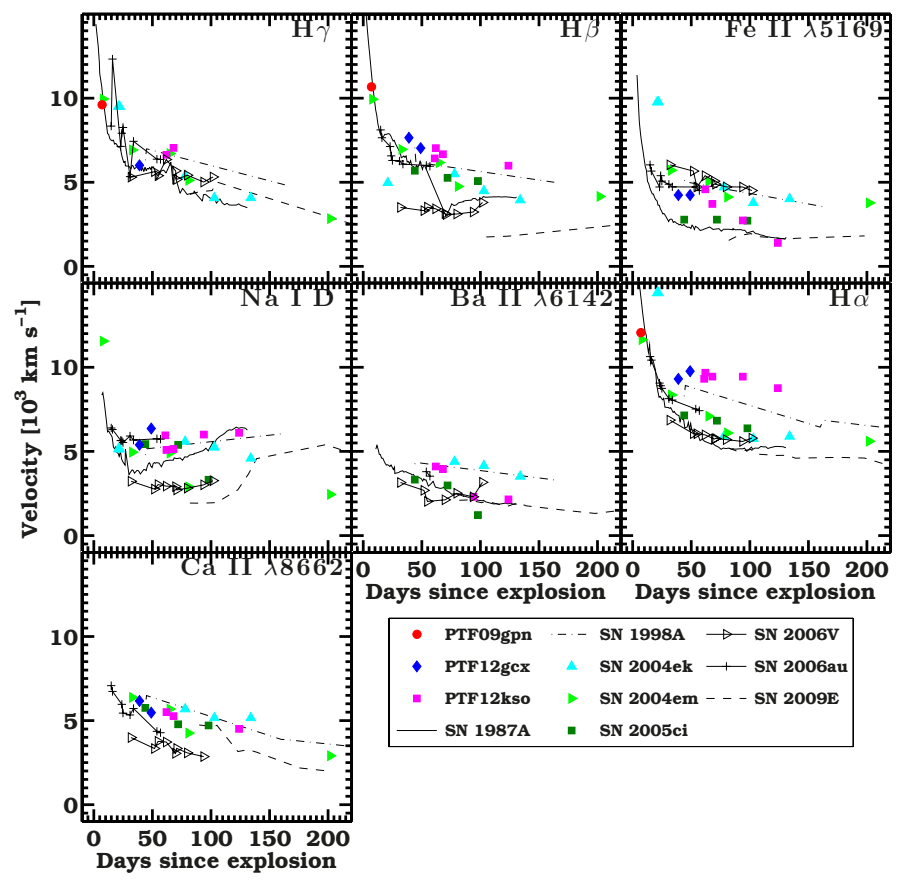

Fig. 11. Velocity evolution from the P-Cygni absorption minima of the most important spectral lines for our PTF and CCCP SNe (colored symbols) as compared to SN 1987A and other long-rising SNe II (black symbols, from the spectra presented by Phillips et al. 1988; Pastorello et al. 2005, 2012; Taddia et al. 2012). Typical uncertainties are of 500$1000 \mathrm{~km} \mathrm{~s}^{-1}$.

\section{Bolometric properties}

For the CCCP and PTF SNe (except for PTF12gcx), we constructed the SEDs after having interpolated the BVRI/Bgri light curves to the same epochs and converted the extinctioncorrected magnitudes into fluxes at the effective wavelength of each filter. The bolometric light curves were computed following the prescriptions by Lyman et al. (2014), to account for the missing near-infrared (NIR) and UV flux: (a) the optical flux was obtained as the trapezoidal integral of the BVRI/Bgri fluxes. (b) The UV flux was computed as the integral of the black-body (BB) fit to the entire SED blueward of the effective $B$-band wavelength during the early cooling phase, and as the trapezoidal integral blueward of the effective $B$-band wavelength with $F_{\lambda}(\lambda \leq 2000 \AA)=0$ at later epochs. The cooling phase coincides with the epochs when the early $B$-band light curve is observed declining. (c) the NIR flux was obtained as the integral of the best $\mathrm{BB}$ fit in the region redward of the effective $I / i$-band wavelength. The BB was fit to the entire SED during the cooling phase, and only to $V R I / \operatorname{gri}(z)$ at later epochs, as the $B$-band flux is potentially affected by line blanketing. The sum of optical, UV, and NIR fluxes was then multiplied by $4 \pi D^{2}$, with $D$ being the distance of the $\mathrm{SN}$, to obtain the bolometric luminosity $\left(L_{\mathrm{Bol}}\right)$. This is shown in Fig. 12 (top panel). In the case of SN $2005 \mathrm{ci}$, we verified that obtaining the bolometric light curve by simply integrating the best BB fit to the entire (BVRI) SED at all epochs would not have changed the result by much, with an average difference in luminosity of $\sim 2 \%$. For this reason, when the $B$ band was missing at late epochs (e.g., SN 2004em), we adopted the integral of the BB fit to VRI as the bolometric flux. For PTF12gcx, where we only have a well-sampled $r$-band light curve, the bolometric light curve was obtained using the bolometric corrections from Lyman et al. (2014) assuming $g-r$ equal to that of SN 1987A (see Fig. 7). 


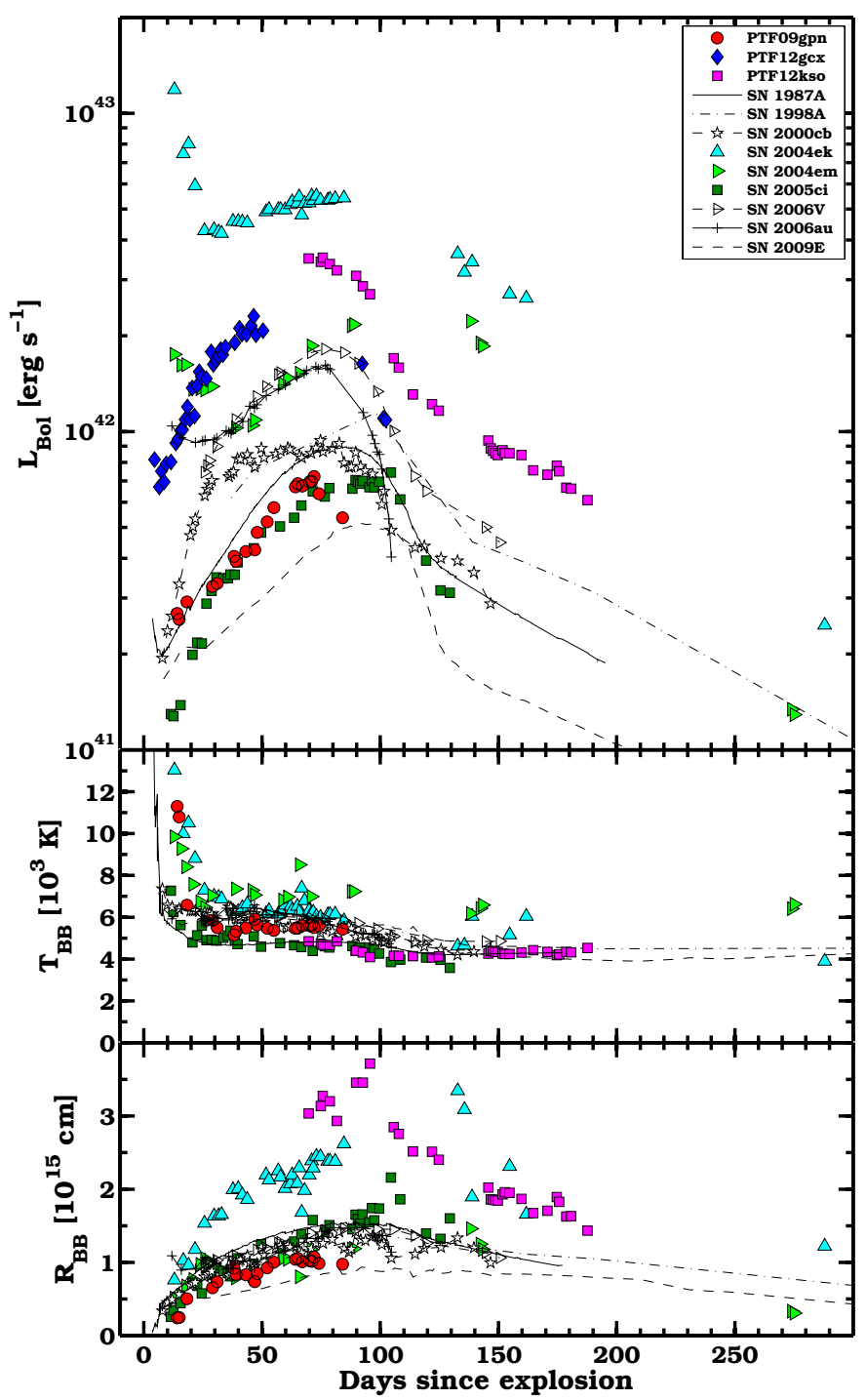

Fig. 12. Bolometric luminosity $\left(L_{\mathrm{Bol}}\right)$, radius $\left(R_{\mathrm{BB}}\right)$, and temperature $\left(T_{\mathrm{BB}}\right)$ evolution from the $\mathrm{BB}$ fit and integration of the SEDs of our $\mathrm{PTF} / \mathrm{CCCP} \mathrm{SNe}$ (colored symbols) and other similar events in the literature (black lines and symbols).

With the $\mathrm{BB}$ fit to the SED we also determined the $\mathrm{BB}$ radius $\left(R_{\mathrm{BB}}\right)$ and the temperature $\left(T_{\mathrm{BB}}\right)$, which are shown in the central and bottom panels of Fig. 12. Again, fitting only VRI/gri or the entire SED does not have a large impact on the final results, with $T_{\mathrm{BB}}$ of SN 2005 ci differing by $3 \%$ on average and by $12 \%$ at most. The radius differs by $6 \%$ on average and by $27 \%$ at most.

With the same method outlined for CCCP and PTF SNe, we also derived $L_{\mathrm{Bol}}, T_{\mathrm{BB}}$, and $R_{\mathrm{BB}}$ for other well-observed SN 1987A-like SNe from the literature (data from Pastorello et al. 2005; Kleiser et al. 2011; Taddia et al. 2012; Pastorello et al. 2012). The results are shown in Fig. 12, where the top panel confirms that among the brightest long-rising SNe II we find SN 2004ek, PTF12kso, and SN 2004em. The faintest are SN 2009E, PTF09gpn, and SN 2005ci. Long rising SNe II from the literature (black lines and symbols) are on average fainter than the SNe in our sample (colored symbols), with SNe 2006V and 2006au being the most luminous. At late epochs, all of the light curves show a decline rate identical to that of SN 1987A, which is consistent with the ${ }^{56} \mathrm{Co}$ decay rate, with the exception of SNe 2004ek and 2004em (which decline faster). SNe 2004ek, 2004em, 2005ci, and PTF09gpn have a decreasing luminosity and a decreasing temperature (central panel of Fig. 12) at early epochs. The temperature profiles are rather similar for all the $\mathrm{SNe}$, with an early cooling (up to $\sim 20 \mathrm{~d}$ ) followed by an almost constant temperature between $\sim 4000$ and $\sim 7000 \mathrm{~K}$ depending on the object. The BB radii (bottom panel of Fig. 12) are on the order of $10^{15} \mathrm{~cm}$, with PTF12kso and SN 2004ek exhibiting the largest values. After a fast expansion, they peak around the epoch of maximum luminosity and then slowly decrease. In summary, our SN sample of long-rising SNe II increases the spread of the observed bolometric properties and exhibits large variety.

\section{Modeling}

\section{1. ${ }^{56} \mathrm{Ni}$ mass from the linear decaying tail of the bolometric light curve}

We can use the late part of the bolometric light curves to estimate the amount of ${ }^{56} \mathrm{Ni}$ ejected in the SN. The light-curve decline at this phase is linear in magnitude and $\sim 1$ mag every 100 days (the decay rate of $\left.{ }^{56} \mathrm{Co}\right)$. As the decay times of ${ }^{56} \mathrm{Ni}$ and ${ }^{56} \mathrm{Co}\left(\tau_{56} \mathrm{Ni}\right.$ and $\tau_{56} \mathrm{Co}$ ) are known, as well as their specific energy generation rates $\left(\epsilon_{56} \mathrm{Ni}\right.$ and $\left.\epsilon_{56} \mathrm{Co}\right)$, it is possible to constrain the ${ }^{56} \mathrm{Ni}$ mass $\left(M_{56} \mathrm{Ni}\right)$ by fitting the following expression to the bolometric light curve:

$$
\begin{aligned}
L_{\mathrm{Bol}}= & M_{56 \mathrm{Ni}}\left[\epsilon_{56} \mathrm{Ni} \exp \left(-t / \tau_{{ }^{56} \mathrm{Ni}}\right)+\epsilon_{56}{ }_{\mathrm{Co}} \tau^{56} \mathrm{Co} /\left(\tau_{56} \mathrm{Co}\right.\right. \\
& \left.\left.-\tau_{{ }^{56} \mathrm{Ni}}\right)\left(\exp \left(-t / \tau^{56} \mathrm{Co}\right)-\exp \left(-t / \tau^{56} \mathrm{Ni}\right)\right)\right] .
\end{aligned}
$$

For PTF12kso, SN 2004em, and SN 2005ci, we can fit at least two epochs on the linear decline, while SN 2004ek was observed only once at late epochs. For PTF09gpn and PTF12gcx, we can set only an upper limit on the ${ }^{56} \mathrm{Ni}$ mass, as our last bolometric epochs coincide with the beginning of the light-curve tails.

For PTF09gpn we also have $r$-band measurements at very late epochs. If we compare the absolute magnitude at these epochs with that of SN 2009E, the two SNe turn out to have similar ${ }^{56} \mathrm{Ni}$ masses. Pastorello et al. (2012) estimated a ${ }^{56} \mathrm{Ni}$ mass of $0.04 M_{\odot}$ from the quasi-bolometric light curve of SN 2009E, which we adopt as the best value for PTF09gpn.

The objects with the largest and smallest ${ }^{56} \mathrm{Ni}$ masses are PTF12kso $\left(0.23 M_{\odot}\right)$ and PTF09gpn $\left(0.04 M_{\odot}\right)$, respectively. All of the results are reported in Table 4.

The uncertainties in these values are mainly affected by the uncertainty in the distance and in the explosion date. There could also be uncertainty related to the host-galaxy extinction. Therefore, we estimate the total error in the ${ }^{56} \mathrm{Ni}$ mass to be at least $10 \%$.

Our sample of long-rising $\mathrm{SNe}$ II extends the range of ${ }^{56} \mathrm{Ni}$ masses inferred for SN 1987A-like SNe (see Table 5 of Pastorello et al. 2012), with PTF12kso being the most ${ }^{56} \mathrm{Ni}$ rich, about four times more than for SN 1987A. When compared to the ${ }^{56} \mathrm{Ni}$ mass distribution of normal SNe II from Hamuy (2003), Inserra et al. (2013), Anderson et al. (2014), and Rubin et al. (2015), it appears that long-rising SNe II produce more ${ }^{56} \mathrm{Ni}$ on average (see Fig. 13). However, finding SN 1987A-like SNe with low ${ }^{56} \mathrm{Ni}$ masses is more difficult than discovering SNe IIP with the same ${ }^{56} \mathrm{Ni}$ masses, as their lower cooling-envelope luminosity at early epochs makes them fainter on the rise. Even accounting for this bias, long-rising SNe II seem to have a different distribution of ${ }^{56} \mathrm{Ni}$ masses as compared to SNe IIP. A likely explanation for this result is that the progenitors of SN 1987Alike events are on average more massive than those of SNe IIP, 
Table 4. Explosion and progenitor parameters.

\begin{tabular}{c|cc|cc|c}
\hline \hline$S N$ & $\begin{array}{c}E^{a} \\
\left(10^{51} \mathrm{erg}\right)\end{array}$ & $\begin{array}{c}M_{\mathrm{ej}}^{a} \\
\left(M_{\odot}\right)\end{array}$ & $\begin{array}{c}M_{56 \mathrm{Ni}}^{t} \\
\left(M_{\odot}\right)\end{array}$ & $\begin{array}{c}\operatorname{mix}_{56 \mathrm{Ni}}^{h} \\
\left(\% / M_{\mathrm{ej}}\right)\end{array}$ & $\begin{array}{c}R^{h} \\
\left(R_{\odot}\right)\end{array}$ \\
\hline 2004ek & 9.3 & 28 & 0.217 & 41 & 2303 \\
2004em & 11.3 & 43 & 0.102 & 25 & 316 \\
2005ci & 2.3 & 19 & 0.065 & 90 & 33 \\
PTF09gpn & 1.0 & 12 & 0.040 & 35 & 120 \\
PTF12gcx & 3.6 & 12 & $<0.181$ & 100 & 346 \\
PTF12kso & 3.4 & 17 & 0.230 & 90 & $\ldots$ \\
\hline
\end{tabular}

Notes. ${ }^{(a)}$ From Arnett et al. (1989). ${ }^{(t)}$ From the modeling of the bolometric light curve tail. ${ }^{(h)}$ From the hydrodynamical models. Errors in the ${ }^{56} \mathrm{Ni}$ mass are mainly due to the uncertainty in the distance and in the explosion epoch. We estimate the total uncertainty to be $\sim 10 \%$ for each SN. The ${ }^{56} \mathrm{Ni}$ mass for PTF09gpn is determined by the $r$-band light-curve comparison to SN 2009E at late epochs.

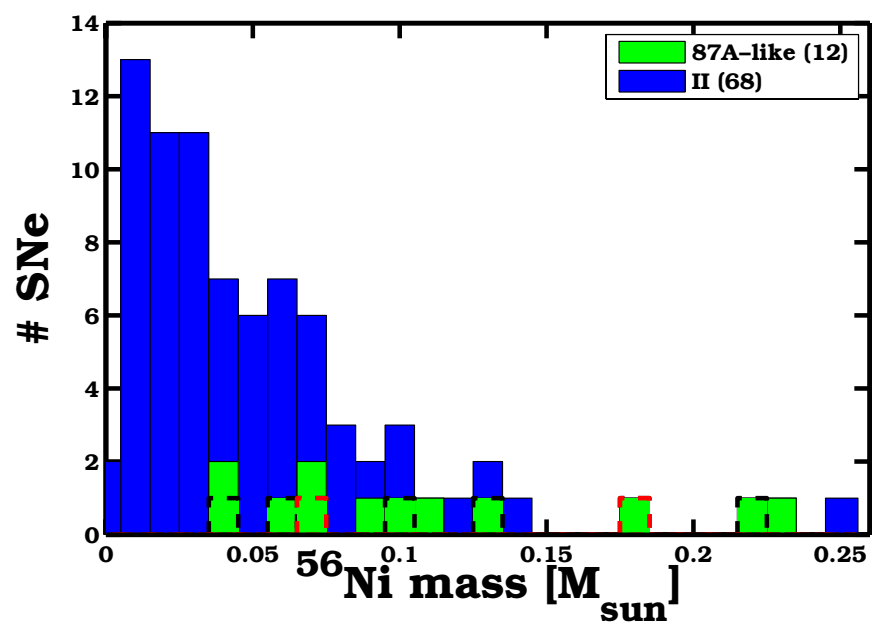

Fig. 13. ${ }^{56} \mathrm{Ni}$ mass distribution for SNe II (IIP and IIL, estimates from Hamuy 2003; Inserra et al. 2013; Anderson et al. 2014; and Rubin et al. 2015 ) in blue, and our SN 1987A-like SNe in green. ${ }^{56} \mathrm{Ni}$ mass estimates that are based on at most two points on the radioactive tail are highlighted by dashed edges (red lines indicate upper limits, black lines mark lower limits).

and therefore they also produce more ${ }^{56} \mathrm{Ni}$, as these two quantities show a correlation (see, e.g., Utrobin \& Chugai 2011). We also notice that for seven SN 1987A-like events the ${ }^{56} \mathrm{Ni}$ mass is derived from at most two points on the radioactive tail. This might introduce a bias in the ${ }^{56} \mathrm{Ni}$ mass estimates if gamma-rays are escaping.

Based on the ${ }^{56} \mathrm{Ni}$ mass estimates from the tail of the bolometric light curves of our SNe and other SN 1987A-like SNe, we can conclude that the amount of synthesized ${ }^{56} \mathrm{Ni}$ in longrising SNe II can vary widely, between $0.04 M_{\odot}$ and $0.23 M_{\odot}$. The lower value is consistent with those of normal SNe IIP such as SN 1999em, the higher one with those of luminous SNe IIP (e.g., SN 1992af; Nadyozhin 2003) or moderately faint SNe Ibc (e.g., Taddia et al. 2015a). In Fig. 14 we can see how our sample (colored symbols) extends the range of ${ }^{56} \mathrm{Ni}$ masses up to $0.23 M_{\odot}$ compared to the previously known long-rising SNe II.

\subsection{Explosion energy and ejecta mass from scaling relations}

In order to estimate the explosion energy $(E)$ and the ejecta mass $\left(M_{\mathrm{ej}}\right)$, we need to combine the information about the bolometric rise time and the photospheric velocity, as done by Taddia et al. (2012). For radioactively powered SNe, the diffusion time given by Arnett (1979) is $t_{\mathrm{d}}=\left(\kappa M_{\mathrm{ej}} / v_{\mathrm{ph}}\right)^{1 / 2}$, and

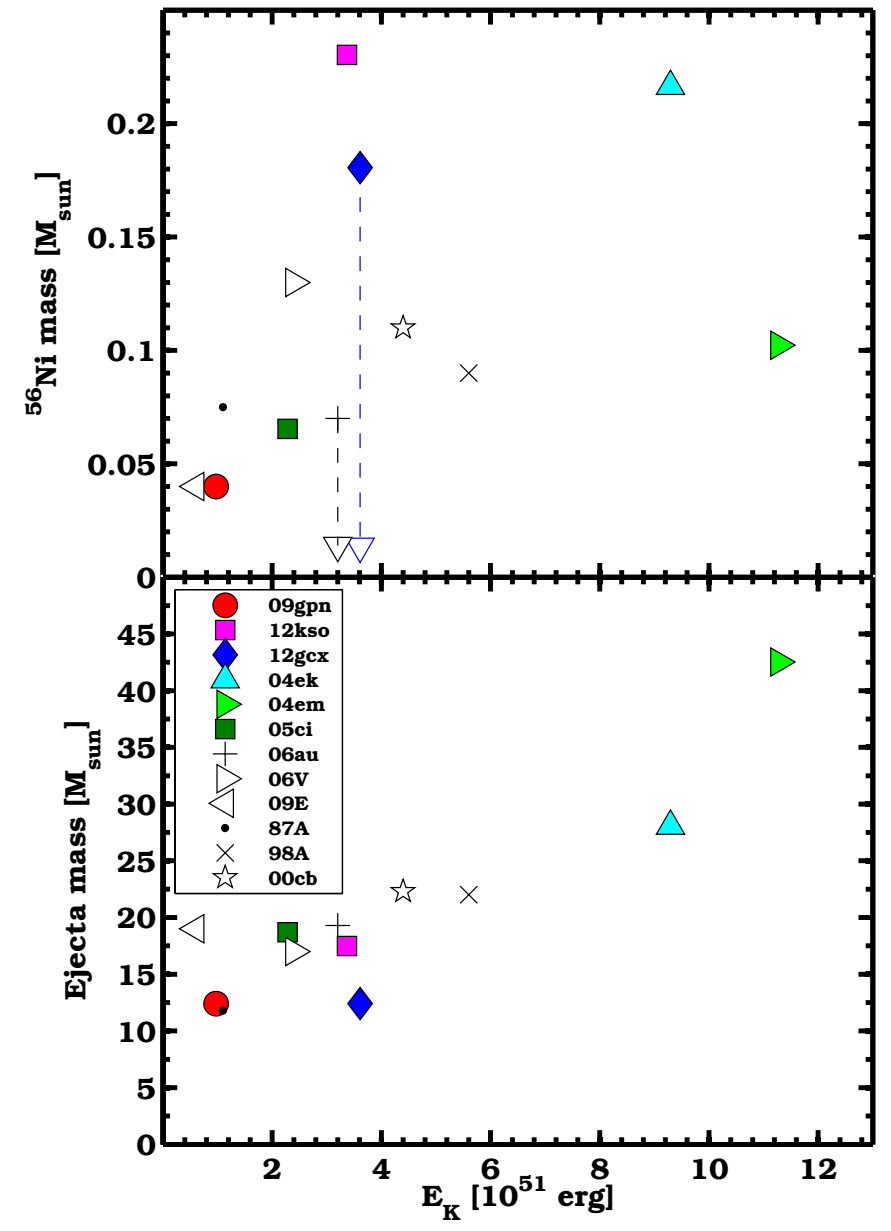

Fig. 14. ${ }^{56} \mathrm{Ni}$ mass and ejecta mass versus explosion energy for our PTF and CCCP SNe and for other well-observed SN 1987A-like SNe (Taddia et al. 2012). In the top panel, dashed vertical arrows are limits on the ${ }^{56} \mathrm{Ni}$ mass.

$E \propto M_{\mathrm{ej}} v_{\mathrm{ph}}^{2}$. We assume the same mean opacity $\kappa$ for all our SNe, and adopt the Fe II $\lambda 5169$ velocity (see Fig. 11) around peak as $v_{\text {ph }}$ (for PTF09gpn we adopted the velocity of SN 1987A) and the bolometric rise time as $t_{\mathrm{d}}$. Using values from SN 1987A, $E(87 \mathrm{~A})=1.1 \times 10^{51} \mathrm{erg}, M_{\mathrm{ej}}(87 \mathrm{~A})=14 M_{\odot}($ Blinnikov et al. $2000), t_{\mathrm{d}}(87 \mathrm{~A})=84 \mathrm{~d}$, and $v_{\mathrm{ph}}(87 \mathrm{~A})=2200 \mathrm{~km} \mathrm{~s}^{-1}$, we obtain estimates of $E$ and $M_{\mathrm{ej}}$ for the other SNe by scaling. These are listed in Table 4.

The long rise times and fast expansion velocities of SNe 2004ek and 2004em imply large ejecta masses 
$\left(\sim 30-40 M_{\odot}\right)$ and energies $\left(\sim 10^{52} \mathrm{erg}\right)$. Lower values characterize the other $\mathrm{SNe}$, which however show larger ejecta masses and energies than SN 1987A, with the exception of PTF09gpn. The $\mathrm{SNe}$ observed in our sample are found to be more energetic and have larger masses (on average) than the old sample, as shown in Fig. 14. It appears from the same figure that the ejecta mass and the explosion energy are correlated, as well as the ${ }^{56} \mathrm{Ni}$ mass and the explosion energy. The estimates of $E$ and $M_{\mathrm{ej}}$ are based on simple scaling relations and therefore must be taken with caution. We will show in Sect. 8.3 that the rise times of hydrodynamical models show a more complicated dependence on the progenitor parameters as compared to what we used here, including a dependence on the degree of ${ }^{56} \mathrm{Ni}$ mixing and on the ${ }^{56} \mathrm{Ni}$ mass.

\subsection{Hydrodynamical modeling}

In Figs. 1 and 12 we showed how the early-time shape of our long-rising $\mathrm{SNe}$ II is different for the different events. It is known, also from analytic modeling (see, e.g., Chevalier \& Fransson 2008), that SN progenitor radii influence the early SN emission, with the more luminous early light curves corresponding to the SNe with larger progenitors.

Here we aim to demonstrate that we need progenitor radii between that of a RSG ( $\gtrsim 500 R_{\odot}$, typical of SN IIP progenitors) and that of a compact BSG $\left(\lesssim 100 R_{\odot}\right.$, progenitor of a canonical SN 1987A-like SN) to explain the early light-curve dispersion of these objects. To do this, we made use of open-source codes to evolve massive and hydrogen-rich progenitor stars and explode them, producing bolometric light curves that were compared to our observations.

We used the Modules for Experiments in Stellar Astrophysics (MESA; Paxton et al. 2011) to evolve $M_{\text {ZAMS }}=15,20,25$, and $30 M_{\odot}$ stars with different metallicities (from $Z=10^{-5}$ to $Z=5 \times 10^{-3}$ ) and rotation velocities $\left(v_{\text {rot }}=0-600 \mathrm{~km} \mathrm{~s}^{-1}\right)$, until they end their lives at different positions in the HR diagram. We thus constructed a sequence of models with stellar radii from $\sim 33$ to $\sim 1071 R_{\odot}$ for stars with $M_{\text {ZAMS }}=20 M_{\odot}$. We decided to produce stars with compact radii for the other initial masses $\left(15,25\right.$, and $\left.30 M_{\odot}\right)$. We prohibited mass loss to preserve a large $\mathrm{H}$ envelope and did not include overshooting in our computations. The HR diagram including our models is shown in Fig. 15. Low metallicity and fast rotation favor the production of BSG stars in the HR diagram, whereas high metallicity and low rotation velocity produce RSG stars as SN progenitors (e.g., Podsiadlowski 1992).

The aim of this exercise was to construct a sequence of similar $M_{\text {ZAMS }}$ stars of different final radii to use as input for the SuperNova Explosion Code (SNEC; Morozova et al. 2015), in order to produce bolometric light curves and analyze the effect of the different parameters on the SN light curves. We produced a set of hydrodynamical models with a range of progenitor radii $(R)$, explosion energies $(E)$, final masses $\left(M=M_{\text {ZAMS }}=\right.$ $M_{\text {ej }}+1.4 M_{\odot}$, as we inhibited the mass loss and consider the formation of a central compact object of $\left.1.4 M_{\odot}\right),{ }^{56} \mathrm{Ni}$ masses $\left(M_{56} \mathrm{Ni}\right)$, and degrees of ${ }^{56} \mathrm{Ni}$ mixing $\left(\right.$ mix $\left._{56} \mathrm{Ni}\right)$. The degree of ${ }^{56} \mathrm{Ni}$ mixing is expressed as a fraction of the final mass, and the ${ }^{56} \mathrm{Ni}$ is uniformly distributed in the region where it is present. For example, a mix ${ }_{56} \mathrm{Ni}$ of 0.9 implies that the ${ }^{56} \mathrm{Ni}$ mass is uniformly distributed up to the radius that includes $90 \%$ of the final progenitor mass.

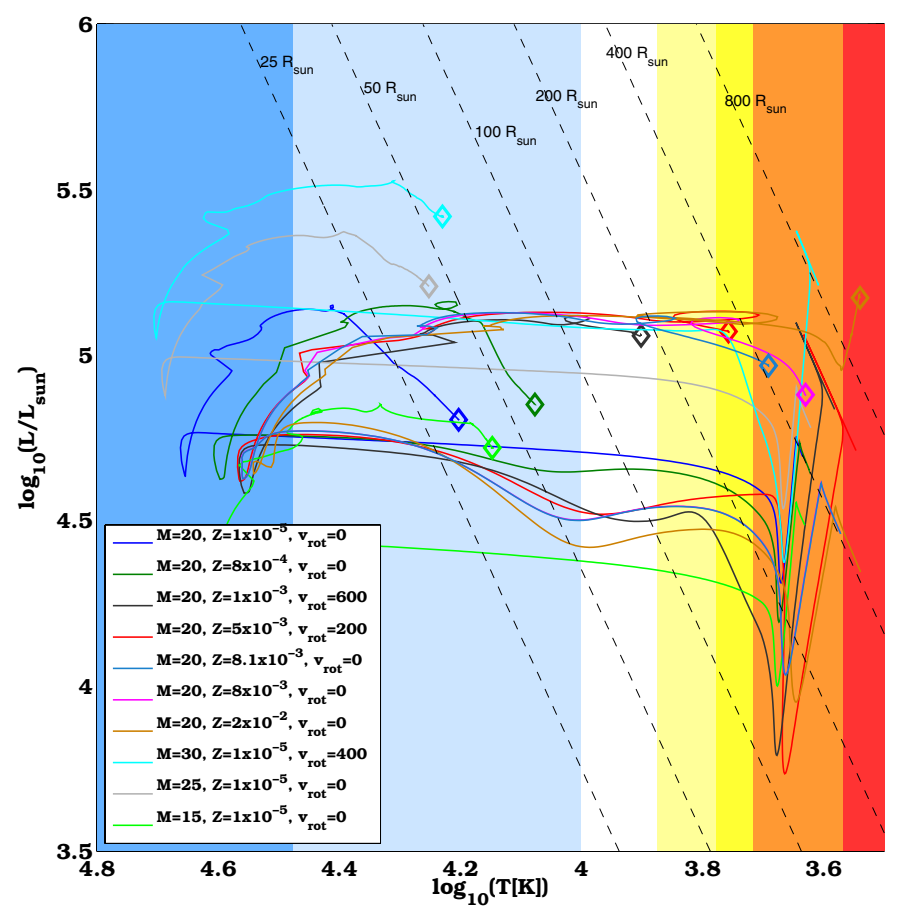

Fig. 15. HR diagram for a series of stellar models obtained with MESA. Different metallicities and rotation velocities (see the legend) are used to create a sequence of $\mathrm{SN}$ progenitors with progressively larger radii (see the diamond symbols as compared to the dashed isoradius lines).

If we keep the same mass $\left(M=20 M_{\odot}\right)$, the same ${ }^{56} \mathrm{Ni}$ mass $\left(0.075 M_{\odot}\right)$, and the same explosion energy $\left(10^{51} \mathrm{erg}=1 \mathrm{~B}\right)$, we can see in Fig. 16 (top-left panel) that for compact radii, the light curve is very similar to that of SN 1987A. For larger radii, the luminosity of the early part (before the light-curve rise) becomes larger, until the SN light curve reaches that of a normal SN IIP. This occurs for radii $\gtrsim 420 R_{\odot}$ for our given parameter set. The same result was found by Young (2004). For large radii, the decline in luminosity is also slower than for small radii.

In Fig. 16 (top-central panel) we show that, for a range of final masses from 15 to $30 M_{\odot}$, if we keep the same ratio between $E$ and $M$, as well as approximately the same $R$, then the rise time and the early luminosity of the light curves are generically very similar.

The top-right panel of Fig. 16 shows that for the same $M=$ $20 M_{\odot}, M_{56} \mathrm{Ni}=0.075 M_{\odot}$, and $R=33 R_{\odot}$, we obtain a brighter and earlier peak for larger energies (see also Young 2004).

For larger ${ }^{56} \mathrm{Ni}$ mass (keeping the same $M=20 M_{\odot}, R=$ $33 R_{\odot}$, and $\left.E=10^{51} \mathrm{erg}=1 \mathrm{~B}\right)$, the rise time is longer; for larger ${ }^{56} \mathrm{Ni}$ mixing it is shorter (bottom panels of Fig. 16). The effects of ${ }^{56} \mathrm{Ni}$ mixing on the peak was also investigated by Young (2004).

As we could not produce all the models needed to scan the entire space of the progenitor parameters, we made use of our set of models to derive scaling relations for the time of the peak, luminosity at $+10 \mathrm{~d}$, and time of the rise. This allowed us to constrain the degree of ${ }^{56} \mathrm{Ni}$ mixing, $E / M$, and the progenitor radius for our $\mathrm{SNe}$.

We measured the peak epochs of these models and we investigated how they change depending on each progenitor parameter. We found that

$t_{\text {peak }} \propto(E / M)^{-0.4} M_{56 \mathrm{Ni}}^{0.2} \operatorname{mix}_{56}^{-0.25}$. 

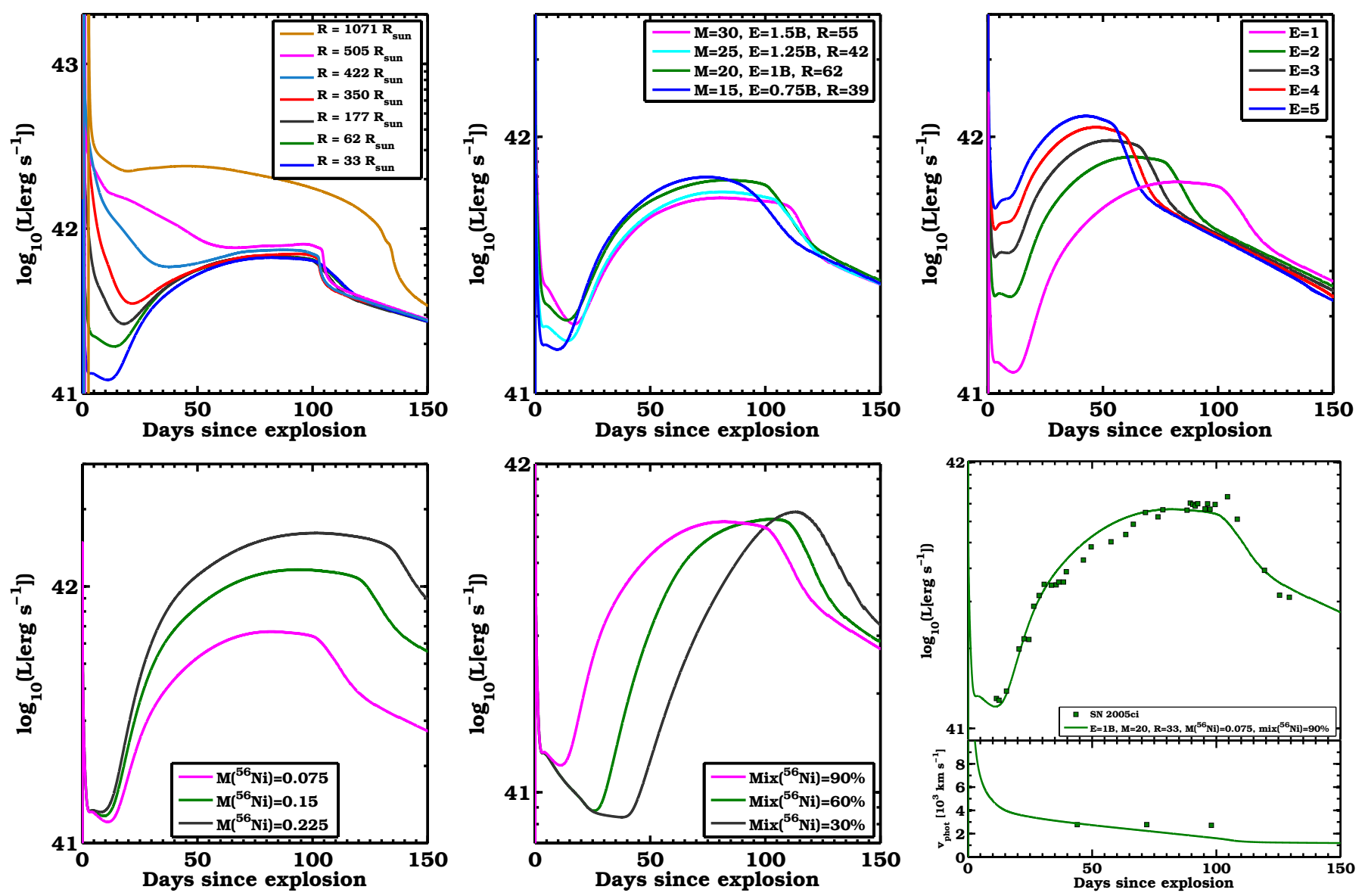

Fig. 16. SNEC bolometric light curves of the SNe from the progenitor stars obtained from the stellar models shown in Fig. 15. We tested how the light curves change depending on the different progenitor and explosion parameters. In the bottom-right panel we show one of our models fitting the bolometric light curve and the velocity profile of SN $2005 \mathrm{ci}$.

The luminosity of the early part (measured at $10 \mathrm{~d}$ after explosion) scales approximately like

$L_{+10 \mathrm{~d}} \propto(E / M)^{0.9} R^{0.7}$

for $R \lesssim 350 R_{\odot}$. The time at which the light curve starts to rise $\left(t_{\mathrm{sr}}\right)$ after the early cooling depends mainly on the degree of ${ }^{56} \mathrm{Ni}$ mixing as

$t_{\mathrm{sr}} \propto \operatorname{mix}_{56 \mathrm{Ni}}^{-0.9}$.

We also obtained estimates of the progenitor radius from these relations by scaling with the parameters of the hydrodynamical model of SN 2005ci, shown in the bottom-left panel of Fig. 16. The bolometric light curve and the photospheric velocity of SN 2005ci are well reproduced by a model with $M=20 M_{\odot}$, $R=33 R_{\odot}, E=1 \mathrm{~B}, M_{56} \mathrm{Ni}=0.075 M_{\odot}$, and $\operatorname{mix}_{56}{ }^{\mathrm{Ni}}=90 \%$. The light curve of SN 2005ci is rather similar to that of SN 2000cb (Kleiser et al. 2011), which was modeled by Utrobin \& Chugai (2011), who found a similar mass $\left(M_{\mathrm{env}}=22.3 M_{\odot}\right)$ and progenitor radius $\left(R=35 R_{\odot}\right)$, but higher energy $(E=4.4 \mathrm{~B})$.

For each of our SNe we make use of the $M^{56}{ }^{\mathrm{Ni}}$ measured in Sect. 8.1. We measure the epoch of the light-curve rise $\left(t_{\mathrm{sr}}\right)$ to assess the degree of mix ${ }^{6} \mathrm{Ni}_{\mathrm{i}}$ via Eq. (4) and scaling to SN $2005 \mathrm{ci}$. By measuring the rise time we make use of Eq. (2) to estimate $E / M$ by scaling to SN 2005 ci. Then we can use Eq. (3) and the obtained values of $E / M$ to estimate the radius by scaling to SN 2005ci.

In Table 4 we report the parameter estimates obtained from these scaling relations. We obtain radii of about $320-350 R_{\odot}$ for
SN 2004em and PTF12gcx, of $\sim 120 R_{\odot}$ for PTF09gpn, and of a few thousands $R_{\odot}$ for SN 2004ek. Therefore, we suggest that we have observed $\mathrm{H}$-rich $\mathrm{SNe}$ with progenitor radii that are intermediate between those of RSGs and BSGs, implying that H-rich stars in the A to K spectral classes can also explode and become $\mathrm{SNe}$ II. Moreover, we found that there is a large variety in the degree of ${ }^{56} \mathrm{Ni}$ mixing, ranging from $25 \%$ to $100 \%$ of the final mass.

\section{Discussion}

In this work we have added three new and well-observed longrising SNe II from PTF and analyzed three more SNe from CCCP. These objects are an important addition to this $\mathrm{SN}$ class as long-rising SNe II are extremely rare events. Pastorello et al. (2012) estimated that SN 1987A-like SNe make up 1$3 \%$ of CC SNe based on the data from the Asiago Supernova Catalogue. Also, Kleiser et al. (2011) derived a similar value based on the data from the Lick Observatory SN Search (Filippenko et al. 2001; Leaman et al. 2011; Li et al. 2011a,b). If we consider the CCCP SN program, which observed nearby CC SNe, three SN 1987A-like SNe (from a total of 62 CC $\mathrm{SNe}$ ) were followed, suggesting a fraction of $5 \%$. The PTF/iPTF survey is better suited to estimate the rate as it is not a targeted search. Within a distance modulus of $\mu \approx 35 \mathrm{mag}$, we found only PTF09gpn out of a total of $108 \mathrm{CC}$ SNe observed within the same volume. This suggests a fraction of $1 \%$, consistent with the value suggested by Pastorello et al. (2012). 
The progenitor parameters suggest stars with a radius smaller than that of a typical RSG, which is $\gtrsim 500 R_{\odot}$ (with the exception of SN 2004ek).

We estimated the progenitor radii by using hydrodynamical models. In particular, we obtained a good fit to the bolometric luminosity and photospheric velocity of SN 2005ci. Thereafter, using the hydrodynamical models, we derived scaling relations for the peak epoch, the early luminosity, and the epoch of the lightcurve rise, which depends on $R, E / M, M_{56} \mathrm{Ni}$, and mix ${ }_{56} \mathrm{Ni}$. We confirmed that the early luminosity depends on the ratio $E / M$ and on the $R$ with similar power-law indexes. However, compared to the scaling relation based on the diffusion time from the model of Arnett, we found important differences. For instance, we found that $t_{\text {peak }}$ depends on $M / E$ instead of $M^{3} / E$. This was also found by Utrobin (2005) for his set of hydrodynamical models in the envelope-mass range 15-21 $M_{\odot}$. We also considered the dependence of $t_{\text {peak }}$ on $M_{56} \mathrm{Ni}$ and $\operatorname{mix}_{56} \mathrm{Ni}$, which were previously ignored.

With the relations estimated from this series of hydrodynamical models, we obtained progenitor radii of $\sim 30 R_{\odot}$ for SN 2005ci, $\sim 120 R_{\odot}$ for PTF09gpn, and $\sim 320-350 R_{\odot}$ for SN 2004em and PTF12gcx. Considering that the ejecta masses range from $\sim 10$ to $\sim 40 M_{\odot}$, the radii of these stars imply a range of progenitor stars from BSGs (SN 2005ci and PTF09gpn) to yellow supergiants (YSGs; e.g., SN 2004em and PTF12gcx), as can be seen in the HR diagram of Taddia et al. (2012) and in Fig. 15.

For SN 2004ek, we obtained a large progenitor radius of a few thousand $R_{\odot}$, typical of a RSG. Its bolometric light curve shows a late-time peak, but the rise occurring after the initial luminosity drop is very shallow compared to that of SN 1987A, and the early-time light curve is very bright. We can interpret the light curve of SN 2004ek as the combination of radioactive emission from its large ${ }^{56} \mathrm{Ni}$ mass (important only at peak and even more at later epochs) and cooling emission from its large envelope (dominating the early epochs).

For two host galaxies of our PTF SNe (PTF09gpn and PTF12kso), we have measured very low metallicity $(Z \approx$ $1 / 10 Z_{\odot}$ ), lower than the metallicity measured in the Small Magellanic Cloud (SMC). A low metallicity $\left(\lesssim 1 / 3 Z_{\odot}\right)$ can favor a blue solution in the HR diagram for a single star, with the star exploding as a BSG (see Podsiadlowski 1992 and Weiss 1989). In this case, the SN progenitors could have spent their entire lives in the BSG stage, and did not have a RSG phase such as that of SN 1987A. These two SNe confirm the trend of longrising SNe II at low metallicity found by Taddia et al. (2013).

\section{Conclusions}

- We have added and analyzed six new long-rising SNe II to this rare family, three from PTF and three from CCCP. These objects present similarities in the light curves, but also interesting differences, especially at early epochs.

- Our SN sample expands the range of ${ }^{56} \mathrm{Ni}$ masses, explosion energies, and ejecta masses for the long-rising SNe II.

- The radii and the large ejecta masses found for our SNe suggest a BSG to YSG origin for these events. Canonical $\mathrm{SN}$ 1987A-like SNe arise from compact BSGs (e.g., SN 2005ci), while SNe with more luminous early phases arise from stars with a radius of a few hundred $R_{\odot}$ (e.g., SN 2004em). We also found that SN 2004ek comes from an even more extended RSG progenitor star, but shows a longrising light curve owing to the large amount of ejected ${ }^{56} \mathrm{Ni}$.
- We found PTF09gpn and PTF12kso at the lowest host metallicities ever estimated for long-rising SNe II, confirming that low host metallicity is an important ingredient for producing peculiar SNe II, whose radii are typically shorter than those of normal SN IIP progenitors.

Acknowledgements. We thank the staffs of the various observatories (Palomar, Lick, Keck, etc.) where data for this study were obtained. The Oskar Klein Centre is funded by the Swedish Research Council. We gratefully acknowledge the support from the Knut and Alice Wallenberg Foundation. A.G.-Y. is supported by the EU/FP7 via ERC grant No. 307260, the Quantum Universe I-Core program by the Israeli Committee for Planning and Budgeting and the ISF; by Minerva and ISF grants; by the Weizmann-UK "making connections" program; and by Kimmel and ARCHES awards. A.V.F.'s research is supported by the Christopher R. Redlich Fund, the TABASGO Foundation, and NSF grant AST-1211916. D.C.L. and S.F.A. acknowledge support from NSF grants AST-1009571 and AST-1210311, under which part of this research (photometry data collected at MLO) was carried out. We thank Joseph Fedrow, Alyssa Del Rosario, Chuck Horst, and David Jaimes for assistance with the MLO observations. J.M.S. is supported by an NSF Astronomy and Astrophysics Postdoctoral Fellowship under award AST-1302771. This research has made use of the APASS database, located at the AAVSO web site; funding for APASS has been provided by the Robert Martin Ayers Sciences Fund. Research at Lick Observatory is partially supported by a generous gift from Google. Some of the data presented herein were obtained at the W. M. Keck Observatory, which is operated as a scientific partnership among the California Institute of Technology, the University of California, and NASA; the observatory was made possible by the generous financial support of the W. M. Keck Foundation. We acknowledge contributions to CCCP by S. B. Cenko, D. Fox, D. Sand, and A. Soderberg. We acknowledge M. Sullivan and K. Sharon for helping with the CCCP spectral observations. LANL participation in IPTF is supported by the US Department of Energy as part of the Laboratory Directed Research and Development program.

\section{References}

Ahn, C. P., Alexandroff, R., Allende Prieto, C., et al. 2014, ApJS, 211, 17 Amorín, R., Pérez-Montero, E., Contini, T., et al. 2015, A\&A, 578, A105 Anderson, J. P., González-Gaitán, S., Hamuy, M., et al. 2014, ApJ, 786, 67 Arcavi, I., Gal-Yam, A., Kasliwal, M. M., et al. 2010, ApJ, 721, 777 Arcavi, I., Gal-Yam, A., Cenko, S. B., et al. 2012, ApJ, 756, L30 Armstrong, M. 2004, Central Bureau Electronic Telegrams, 82, 1 Arnett, W. D. 1979, ApJ, 230, L37

Arnett, W. D., Bahcall, J. N., Kirshner, R. P., \& Woosley, S. E. 1989, ARA\&A, 27, 629

Asplund, M., Grevesse, N., Sauval, A. J., \& Scott, P. 2009, ARA\&A, 47, 481 Ben-Ami, S., Gal-Yam, A., Filippenko, A. V., et al. 2012, ApJ, 760, L33 Blinnikov, S., Lundqvist, P., Bartunov, O., Nomoto, K., \& Iwamoto, K. 2000, ApJ, 532, 1132

Brown, T. M., Baliber, N., Bianco, F. B., et al. 2013, PASP, 125, 1031

Cao, Y., Kasliwal, M. M., Arcavi, I., et al. 2013, ApJ, 775, L7

Cardamone, C., Schawinski, K., Sarzi, M., et al. 2009, MNRAS, 399, 1191

Cenko, S. B., Fox, D. B., Moon, D.-S., et al. 2006, PASP, 118, 1396

Chevalier, R. A. 1992, ApJ, 394, 599

Chevalier, R. A., \& Fransson, C. 2008, ApJ, 683, L135

Cid Fernandes, R., Mateus, A., Sodré, L., Stasińska, G., \& Gomes, J. M. 2005, MNRAS, 358, 363

Dessart, L., \& Hillier, D. J. 2005, A\&A, 439, 671

Elmhamdi, A., Danziger, I. J., Chugai, N., et al. 2003, MNRAS, 338, 939

Faber, S. M., Phillips, A. C., Kibrick, R. I., et al. 2003, Proc. SPIE, 4841, 1657 Filippenko, A. V. 1997, ARA\&A, 35, 307

Filippenko, A. V., Li, W. D., Treffers, R. R., \& Modjaz, M. 2001, in SmallTelescope Astronomy on Global Scales, eds. W. P. Chen, C. Lemme, \& B. Paczyński (San Francisco: ASP), 246, 121

Filippenko, A. V., Ganeshalingam, M., \& Swift, B. J. 2004, IAU Circ., 8411, 1 Fitzpatrick, E. L. 1999, PASP, 111, 63

Gal-Yam, A. 2012, Science, 337, 927

Gal-Yam, A., Cenko, S. B., Fox, D. B., et al. 2007, in AIP Conf. Ser., Vol. 924, The Multicolored Landscape of Compact Objects and Their Explosive Origins, 924, 297

Gal-Yam, A., Ben-Ami, S., Arcavi, I., et al. 2012, The Astronomer's Telegram, 4293, 1

Gal-Yam, A., Arcavi, I., Ofek, E. O., et al. 2014, Nature, 509, 471

Gall, E. E. E., Polshaw, J., Kotak, R., et al. 2015, A\&A, 582, A3

Gilmozzi, R., Cassatella, A., Clavel, J., et al. 1987, Nature, 328, 318

González-Gaitán, S., Tominaga, N., Molina, J., et al. 2015, MNRAS, 451, 2212

Hamuy, M. 2003, ApJ, 582, 905 
Inserra, C., Pastorello, A., Turatto, M., et al. 2013, A\&A, 555, A142 Kennicutt, R. C., \& Evans, N. J. 2012, ARA\&A, 50, 531

Kewley, L. J., \& Ellison, S. L. 2008, ApJ, 681, 1183

Kleiser, I. K. W., Poznanski, D., Kasen, D., et al. 2011, MNRAS, 415, 372

Komatsu, E., Dunkley, J., Nolta, M. R., et al. 2009, ApJS, 180, 330

Kulkarni, S. R. 2013, The Astronomer's Telegram, 4807, 1

Jaskot, A. E., \& Oey, M. S. 2013, ApJ, 766, 91

Jones, M. I., Hamuy, M., Lira, P., et al. 2009, ApJ, 696, 1176

Larsson, J., Fransson, C., Östlin, G., et al. 2011, Nature, 474, 484

Leaman, J., Li, W., Chornock, R., \& Filippenko, A. V. 2011, MNRAS, 412, 1419

Leloudas, G., Schulze, S., Krühler, T., et al. 2015, MNRAS, 449, 917

Li, W., Leaman, J., Chornock, R., et al. 2011a, MNRAS, 412, 1441

Li, W., Leaman, J., Chornock, R., et al. 2011b, MNRAS, 412, 1473

Lunnan, R., Chornock, R., Berger, E., et al. 2014, ApJ, 787, 138

Lyman, J. D., Bersier, D., \& James, P. A. 2014, MNRAS, 437, 3848

Madison, D. R., \& Li, W. 2005, Central Bureau Electronic Telegrams, 168, 1

Mahabal, A. A., Drake, A. J., Djorgovski, S. G., et al. 2012, Central Bureau Electronic Telegrams, 3296, 1

McCray, R. 1993, ARA\&A, 31, 175

McGaugh, S. S. 1991, ApJ, 380, 140

Miller, J. S., \& Stone, R. P. S. 1993, Lick Observatory Technical Report 66 (Santa Cruz, CA)

Modjaz, M., Kirshner, R., Challis, P., Matheson, T., \& Calkins, M. 2004, Central Bureau Electronic Telegrams, 86, 1

Modjaz, M., Kirshner, R., Challis, P., \& Calkins, M. 2005, Central Bureau Electronic Telegrams, 169, 1

Monard, L. A. G., Boles, T., Puckett, T., \& Cox, L. 2004, IAU Circ., 8405, 1

Morozova, V., Piro, A. L., Renzo, M., et al. 2015, ApJ, 814, 63

Nadyozhin, D. K. 2003, MNRAS, 346, 97

Ofek, E. O., Laher, R., Law, N., et al. 2012, PASP, 124, 62

Oke, J. B., \& Gunn, J. E. 1982, PASP, 94, 586

Oke, J. B., Cohen, J. G., Carr, M., et al. 1995, PASP, 107, 375

Osterbrock, D. E. 1989, Astrophysics of Gaseous Nebulae and Active Galactic

Nuclei, 2nd edn. (Mill Valley, CA: University Science Books)
Pastorello, A., Baron, E., Branch, D., et al. 2005, MNRAS, 360, 950

Pastorello, A., Pumo, M. L., Navasardyan, H., et al. 2012, A\&A, 537, A141

Paxton, B., Bildsten, L., Dotter, A., et al. 2011, ApJS, 192, 3

Pettini, M., \& Pagel, B. E. J. 2004, MNRAS, 348, L59

Phillips, M. M., Heathcote, S. R., Hamuy, M., \& Navarrete, M. 1988, AJ, 95, 1087

Piro, A. L., \& Nakar, E. 2013, ApJ, 769, 67

Podsiadlowski, P. 1992, PASP, 104, 717

Quimby, R. M., Kulkarni, S. R., Kasliwal, M. M., et al. 2011, Nature, 474, 487

Rabinak, I., \& Waxman, E. 2011, ApJ, 728, 63

Rahmer, G., Smith, R., Velur, V., et al. 2008, Proc. SPIE, 7014,

Rau, A., Kulkarni, S. R., Law, N. M., et al. 2009, PASP, 121, 1334

Rubin, A., Gal-Yam, A., De Cia, A., et al. 2015, ApJ, submitted [arXiv: 1512.00733]

Schlafly, E. F., \& Finkbeiner, D. P. 2011, ApJ, 737, 103

Smith, C. E., \& Nelson, B. 1969, PASP, 81, 74

Smith, N., Mauerhan, J. C., Cenko, S. B., et al. 2015, MNRAS, 449, 1876

Sternberg, A., El-Hage, P., Elias-Rosa, N., et al. 2012, The Astronomer's Telegram, 4493, 1

Taddia, F., Stritzinger, M. D., Sollerman, J., et al. 2012, A\&A, 537, A140

Taddia, F., Sollerman, J., Razza, A., et al. 2013, A\&A, 558, A143

Taddia, F., Sollerman, J., Leloudas, G., et al. 2015a, A\&A, 574, A60

Taddia, F., Sollerman, J., Fremling, C., et al. 2015b, A\&A, 580, A131

Taubenberger, S., Pastorello, A., Mazzali, P. A., et al. 2006, MNRAS, 371, 1459

Tomasella, L., Benetti, S., Cappellaro, E., et al. 2012, Central Bureau Electronic Telegrams, 3296, 2

Tsvetkov, D. Y. 2008, Peremennye Zvezdy, 28, 3

Utrobin, V. P. 2005, Astron. Lett., 31, 806

Utrobin, V. P., \& Chugai, N. N. 2011, A\&A, 532, A100

Utrobin, V. P., Chugai, N. N., \& Botticella, M. T. 2010, ApJ, 723, L89

Weiss, A. 1989, ApJ, 339, 365

Yaron, O., \& Gal-Yam, A. 2012, PASP, 124, 668

Young, T. R. 2004, ApJ, 617, 1233

Young, T. R., \& Branch, D. 1988, Nature, 333, 305 


\section{Appendix A: Additional Tables}

Table A.1. Optical photometry of three CCCP long-rising SNe II.

\begin{tabular}{|c|c|c|c|c|}
\hline $\begin{array}{l}\text { JD-2 } 400000 \\
\text { (days) }\end{array}$ & $\begin{array}{c}B \\
(\mathrm{mag})\end{array}$ & $\begin{array}{c}V \\
(\mathrm{mag})\end{array}$ & $\begin{array}{c}R / \text { unf. } \\
\text { (mag) }\end{array}$ & $\begin{array}{c}I \\
(\mathrm{mag})\end{array}$ \\
\hline \multicolumn{5}{|c|}{ SN 2004ek } \\
\hline $53263.47 *$ & $16.870(0.030)$ & $16.770(0.030)$ & $16.610(0.020)$ & $16.380(0.040)$ \\
\hline 53267.20 & $17.105(0.016)$ & $16.866(0.018)$ & $16.622(0.007)$ & $16.277(0.032)$ \\
\hline $53269.41 *$ & $17.060(0.030)$ & $16.900(0.040)$ & $16.570(0.040)$ & $16.300(0.050)$ \\
\hline 53272.17 & $17.305(0.016)$ & $16.966(0.019)$ & $16.712(0.010)$ & $16.258(0.033)$ \\
\hline 53276.18 & $17.505(0.038)$ & $17.036(0.030)$ & $16.723(0.010)$ & $16.358(0.037)$ \\
\hline 53280.24 & $17.495(0.016)$ & $17.066(0.020)$ & $16.742(0.009)$ & $16.308(0.032)$ \\
\hline 53282.16 & & & & $16.328(0.033)$ \\
\hline 53283.40 & $17.525(0.016)$ & $17.116(0.019)$ & $16.782(0.007)$ & $16.328(0.033)$ \\
\hline 53288.22 & $17.485(0.019)$ & & $16.663(0.018)$ & $16.238(0.034)$ \\
\hline 53290.41 & & \multicolumn{2}{|l|}{$17.116(0.066)$} & $\ldots$ \\
\hline 53292.17 & $17.515(0.020)$ & & $16.642(0.010)$ & \\
\hline $53294.26^{*}$ & $17.570(0.030)$ & $17.030(0.030)$ & $16.610(0.030)$ & $16.250(0.030)$ \\
\hline 53302.18 & $17.555(0.025)$ & $17.016(0.021)$ & & \\
\hline 53303.46 & $17.525(0.019)$ & $16.966(0.027)$ & $16.492(0.007)$ & $16.117(0.037)$ \\
\hline $53307.41 *$ & $17.620(0.060)$ & $16.960(0.040)$ & $16.490(0.030)$ & $16.040(0.030)$ \\
\hline 53308.37 & \multicolumn{2}{|l|}{$17.595(0.070)$} & & \\
\hline 53310.24 & \multirow{3}{*}{$\begin{array}{c}\ldots \\
17.520(0.040)\end{array}$} & $16.896(0.023)$ & $16.483(0.015)$ & $16.087(0.044)$ \\
\hline $53312.51 *$ & & $16.870(0.030)$ & $16.430(0.030)$ & $16.070(0.030)$ \\
\hline 53313.24 & & $16.876(0.018)$ & & $16.008(0.033)$ \\
\hline $53315.45^{*}$ & $17.500(0.040)$ & $16.860(0.020)$ & $16.430(0.020)$ & $16.050(0.050)$ \\
\hline 53316.24 & $17.505(0.019)$ & $16.856(0.021)$ & $16.363(0.007)$ & $15.998(0.032)$ \\
\hline $53317.30 *$ & $17.650(0.030)$ & $16.780(0.020)$ & $16.500(0.020)$ & $16.090(0.050)$ \\
\hline $53318.50 *$ & $17.580(0.040)$ & $16.790(0.030)$ & $16.370(0.040)$ & \\
\hline $53320.45^{*}$ & $17.530(0.020)$ & $16.890(0.020)$ & $16.420(0.020)$ & $16.040(0.040)$ \\
\hline $53321.47 *$ & $17.640(0.030)$ & $16.820(0.020)$ & $16.310(0.010)$ & $15.950(0.040)$ \\
\hline 53322.17 & $17.595(0.018)$ & $16.856(0.019)$ & $16.392(0.007)$ & $15.967(0.033)$ \\
\hline $53323.49^{*}$ & $17.570(0.030)$ & $16.870(0.020)$ & $16.350(0.020)$ & $15.950(0.030)$ \\
\hline 53325.26 & $17.605(0.015)$ & $16.906(0.018)$ & $16.402(0.006)$ & \\
\hline 53328.35 & \multirow[t]{2}{*}{$17.645(0.015)$} & & & $15.957(0.032)$ \\
\hline 53329.25 & & $16.856(0.019)$ & $16.363(0.007)$ & \\
\hline $53331.47 *$ & \multirow[t]{2}{*}{$17.650(0.040)$} & $16.840(0.020)$ & $16.360(0.020)$ & $15.930(0.030)$ \\
\hline 53335.16 & & $16.906(0.027)$ & $16.312(0.026)$ & $\ldots$ \\
\hline $53383.27 *$ & \multicolumn{2}{|l|}{$18.790(0.180)$} & $16.760(0.040)$ & \\
\hline 53386.12 & $18.995(0.051)$ & $17.776(0.029)$ & $16.992(0.014)$ & $16.317(0.046)$ \\
\hline $53389.37 *$ & \multirow[t]{2}{*}{. } & $17.400(0.200)$ & & $\ldots$ \\
\hline $53405.22 *$ & & \multirow{2}{*}{$\begin{array}{l}17.840(0.140) \\
17.710(0.190)\end{array}$} & $17.070(0.070)$ & \\
\hline $53412.26^{*}$ & $\ldots$ & & $17.190(0.140)$ & \\
\hline 53538.45 & $\cdots$ & $21.006(0.657)$ & $19.973(0.091)$ & $19.087(0.540)$ \\
\hline 53539.41 & $\ldots$ & $\ldots$ & $20.023(0.122)$ & $\ldots$ \\
\hline & & SN 2004em & & \\
\hline 53276.17 & $17.870(0.082)$ & $17.647(0.055)$ & $17.503(0.079)$ & $17.382(0.073)$ \\
\hline 53279.14 & $17.930(0.074)$ & $17.647(0.060)$ & $17.483(0.126)$ & $17.432(0.081)$ \\
\hline 53281.13 & $17.900(0.074)$ & $17.577(0.058)$ & $17.353(0.032)$ & $17.082(0.064)$ \\
\hline 53284.14 & $18.140(0.075)$ & $17.617(0.054)$ & $17.453(0.104)$ & $17.252(0.059)$ \\
\hline 53287.10 & & & $17.303(0.053)$ & \\
\hline 53289.17 & $18.270(0.112)$ & $17.607(0.057)$ & & $16.872(0.071)$ \\
\hline 53292.12 & $18.170(0.077)$ & $17.547(0.074)$ & $17.403(0.026)$ & $17.042(0.054)$ \\
\hline 53302.17 & $18.560(0.086)$ & $17.707(0.066)$ & $17.453(0.039)$ & $17.172(0.069)$ \\
\hline 53309.11 & $18.630(0.079)$ & $17.677(0.057)$ & $17.383(0.031)$ & $17.172(0.082)$ \\
\hline 53310.18 & $18.550(0.113)$ & $17.667(0.106)$ & $17.403(0.072)$ & $17.052(0.155)$ \\
\hline 53322.11 & $\ldots$ & $17.387(0.054)$ & $\ldots$ & \\
\hline 53324.11 & $\ldots$ & $\ldots$ & $\ldots$ & $16.592(0.081)$ \\
\hline 53329.14 & $18.210(0.079)$ & $17.117(0.054)$ & $17.013(0.041)$ & $16.682(0.046)$ \\
\hline 53334.14 & $\ldots$ & $\ldots$ & $16.713(0.151)$ & $16.542(0.088)$ \\
\hline 53351.14 & $\ldots$ & $\ldots$ & $16.593(0.035)$ & \\
\hline 53352.11 & $17.830(0.073)$ & $16.897(0.053)$ & $16.583(0.020)$ & $16.402(0.068)$ \\
\hline 53401.55 & $\ldots$ & $17.127(0.154)$ & $16.753(0.179)$ & $16.312(0.106)$ \\
\hline 53405.56 & $\ldots$ & $17.297(0.069)$ & & \\
\hline 53406.54 & $\ldots$ & $\ldots$ & $16.943(0.054)$ & $16.662(0.065)$ \\
\hline 53537.32 & $\ldots$ & $\ldots$ & $19.453(0.717)$ & \\
\hline 53538.37 & $\ldots$ & $20.296(0.153)$ & $19.543(0.689)$ & $20.362(0.983)$ \\
\hline & & SN 2005ci & & \\
\hline $53523.86^{* *}$ & & & $20.160(0.150)$ & \\
\hline $53531.81 * *$ & $\ldots$ & $\ldots$ & $18.780(0.130)$ & \\
\hline $53532.85 * *$ & $\ldots$ & $\ldots$ & $18.750(0.130)$ & $\ldots$ \\
\hline
\end{tabular}

Notes. Besides CCCP photometry from Arcavi et al. (2012), the table includes BVRI photometry from Tsvetkov (2008) (marked by "**" and shifted by $+0.1(B),+0.2(V),+0.2(R)$, and $+0.1(I)$ mag with respect to the original values of Tsvetkov 2008 to match the CCCP photometry) of SN 2004ek and unfiltered photometry of SN 2005ci from Kleiser et al. (2011) (marked by “**”). 
F. Taddia et al.: PTF and CCCP long-rising SNe II

Table A.1. continued.

\begin{tabular}{|c|c|c|c|c|}
\hline $\begin{array}{l}\text { JD-2 } 400000 \\
\text { (days) }\end{array}$ & $\begin{array}{c}B \\
(\mathrm{mag})\end{array}$ & $\begin{array}{c}V \\
(\mathrm{mag})\end{array}$ & $\begin{array}{l}R / \text { unf. } \\
\text { (mag) }\end{array}$ & $\begin{array}{c}I \\
(\mathrm{mag})\end{array}$ \\
\hline $53533.83 * *$ & $\ldots$ & $\ldots$ & $18.760(0.130)$ & $\ldots$ \\
\hline $53534.76 * *$ & & & $18.860(0.130)$ & \\
\hline 53535.18 & $19.340(0.299)$ & 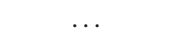 & $18.742(0.107)$ & $18.417(0.329)$ \\
\hline 53536.21 & $19.480(0.280)$ & & $18.662(0.152)$ & $18.117(0.383)$ \\
\hline $53536.80 * *$ & 然 & & $18.510(0.160)$ & \\
\hline 53539.23 & & $19.052(0.138)$ & & $17.877(0.417)$ \\
\hline $53540.82 * *$ & & & $18.310(0.130)$ & \\
\hline 53544.26 & $19.540(0.482)$ & $18.702(0.188)$ & $18.152(0.071)$ & $17.527(0.214)$ \\
\hline $53545.79 * *$ & & & $18.180(0.130)$ & \\
\hline 53546.28 & $19.610(0.182)$ & $18.432(0.186)$ & $17.992(0.079)$ & $17.377(0.259)$ \\
\hline 53548.23 & $19.380(0.277)$ & $18.322(0.177)$ & $17.982(0.049)$ & $17.417(0.258)$ \\
\hline $53549.78 * *$ & & & $17.940(0.130)$ & \\
\hline 53550.18 & $19.000(0.233)$ & & $17.822(0.026)$ & $17.087(0.191)$ \\
\hline 53552.34 & & $18.172(0.156)$ & $17.632(0.050)$ & \\
\hline $53553.79 * *$ & & & $17.750(0.130)$ & \\
\hline 53554.41 & $18.870(0.244)$ & $18.112(0.133)$ & $17.452(0.037)$ & \\
\hline 53557.30 & $19.000(0.227)$ & & & $16.987(0.152)$ \\
\hline $53557.78 * *$ & & & $17.630(0.130)$ & \\
\hline 53559.22 & $19.010(0.192)$ & $17.972(0.115)$ & & $16.967(0.115)$ \\
\hline 53560.26 & $19.070(0.181)$ & $17.822(0.107)$ & $17.422(0.047)$ & $16.847(0.138)$ \\
\hline $53561.76 * *$ & & & $17.720(0.150)$ & \\
\hline 53562.18 & $19.160(0.222)$ & $17.982(0.127)$ & $17.472(0.028)$ & $16.847(0.133)$ \\
\hline 53563.30 & $19.000(0.190)$ & $17.972(0.072)$ & & $16.747(0.110)$ \\
\hline $53565.75^{* *}$ & & & $17.530(0.130)$ & \\
\hline $53567.68 * *$ & & & $17.680(0.150)$ & \\
\hline 53570.19 & $18.990(0.245)$ & $17.682(0.113)$ & $17.262(0.033)$ & $16.597(0.116)$ \\
\hline 53573.30 & & $17.762(0.134)$ & $17.222(0.051)$ & $16.477(0.113)$ \\
\hline 53581.29 & $18.990(0.212)$ & $17.612(0.103)$ & $17.162(0.043)$ & $16.387(0.107)$ \\
\hline 53587.24 & $18.970(0.251)$ & $17.632(0.099)$ & $17.012(0.036)$ & $16.407(0.113)$ \\
\hline 53590.29 & $18.770(0.192)$ & $17.572(0.094)$ & ent & $16.287(0.093)$ \\
\hline 53595.16 & & $17.522(0.091)$ & & $16.137(0.149)$ \\
\hline 53600.21 & $18.840(0.129)$ & $17.452(0.075)$ & $16.922(0.035)$ & $16.177(0.097)$ \\
\hline 53602.18 & $18.990(0.493)$ & $17.422(0.073)$ & $16.802(0.033)$ & $16.137(0.098)$ \\
\hline 53611.93 & $18.760(0.139)$ & $17.422(0.074)$ & $16.792(0.028)$ & $16.177(0.103)$ \\
\hline 53613.23 & $18.930(0.215)$ & $17.442(0.103)$ & & $16.077(0.100)$ \\
\hline 53614.24 & & & $16.782(0.023)$ & \\
\hline 53615.17 & $18.720(0.152)$ & $17.422(0.093)$ & & $16.127(0.116)$ \\
\hline 53616.21 & $18.840(0.252)$ & $17.472(0.098)$ & $16.802(0.033)$ & $16.097(0.102)$ \\
\hline 53619.16 & $18.900(0.197)$ & $17.462(0.082)$ & & $16.147(0.103)$ \\
\hline 53620.18 & $\ldots$ & $17.552(0.056)$ & $\cdots$ & $16.117(0.174)$ \\
\hline 53621.19 & & $17.492(0.092)$ & & $16.157(0.107)$ \\
\hline 53623.23 & & (x) & $16.822(0.047)$ & \\
\hline 53628.20 & $18.920(0.476)$ & & & $16.037(0.125)$ \\
\hline 53632.13 & $19.260(0.356)$ & $17.842(0.203)$ & $17.102(0.033)$ & \\
\hline 53643.11 & $19.240(0.655)$ & $18.332(0.203)$ & $17.532(0.084)$ & $16.787(0.157)$ \\
\hline 53649.15 & & $18.612(0.297)$ & $17.842(0.074)$ & \\
\hline 53653.11 & $19.740(0.430)$ & $19.072(0.349)$ & $17.932(0.078)$ & $17.137(0.202)$ \\
\hline
\end{tabular}


Table A.2. Optical photometry of three PTF SN 1987A-like SNe.

\begin{tabular}{|c|c|c|c|c|c|}
\hline $\begin{array}{l}\text { JD-2 } 400000 \\
\text { (days) }\end{array}$ & $\begin{array}{c}B \\
(\mathrm{mag})\end{array}$ & $\begin{array}{c}g \\
(\mathrm{mag})\end{array}$ & $\begin{array}{c}r \\
(\mathrm{mag})\end{array}$ & $\begin{array}{c}i \\
(\mathrm{mag})\end{array}$ & $\begin{array}{c}z \\
(\mathrm{mag})\end{array}$ \\
\hline \multicolumn{6}{|c|}{ PTF09gpn } \\
\hline $55137.77 *$ & $\ldots$ & $\ldots$ & $20.300(0.017)$ & & $\ldots$ \\
\hline $55142.82 *$ & $\ldots$ & $\ldots$ & $19.560(0.170)$ & $\ldots$ & $\ldots$ \\
\hline $55144.89 *$ & & $\cdots$ & $19.491(0.094)$ & & $\cdots$ \\
\hline 55151.80 & $20.302(0.143)$ & $\ldots$ & $19.181(0.048)$ & $19.248(0.086)$ & $\ldots$ \\
\hline 55152.71 & $\ldots$ & $\ldots$ & $19.238(0.027)$ & $19.290(0.043)$ & $\ldots$ \\
\hline $55152.72 *$ & & & $19.510(0.004)$ & & \\
\hline 55155.89 & $20.585(0.065)$ & $19.873(0.044)$ & $19.286(0.027)$ & $19.238(0.036)$ & $19.446(0.110)$ \\
\hline 55166.78 & $\ldots$ & $19.947(0.129)$ & $19.227(0.054)$ & $19.061(0.065)$ & $19.268(0.133)$ \\
\hline 55168.72 & $\ldots$ & $20.097(0.086)$ & $19.188(0.045)$ & $19.127(0.051)$ & $19.139(0.083)$ \\
\hline $55168.74 *$ & & & $19.557(0.045)$ & & ... \\
\hline 55175.80 & 20.804(0.119) & $20.004(0.056)$ & $19.058(0.036)$ & $18.839(0.034)$ & \\
\hline 55176.73 & $20.576(0.111)$ & $19.997(0.049)$ & $19.046(0.043)$ & $18.949(0.050)$ & $18.704(0.198)$ \\
\hline 55180.80 & $20.584(0.126)$ & $\ldots$ & $18.997(0.042)$ & $18.772(0.042)$ & $18.808(0.096)$ \\
\hline $55181.62 *$ & ... & $\ldots$ & $19.149(0.260)$ & & ... \\
\hline $55183.64 *$ & $\ldots$ & $\ldots$ & $19.153(0.046)$ & & 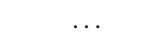 \\
\hline 55184.64 & & $19.615(0.059)$ & $18.997(0.079)$ & $18.697(0.069)$ & \\
\hline 55185.65 & $20.336(0.043)$ & $\ldots$ & $18.801(0.020)$ & $18.669(0.020)$ & $18.847(0.062)$ \\
\hline $55189.60 *$ & 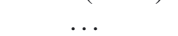 & & $18.798(0.137)$ & & \\
\hline 55189.83 & $20.243(0.121)$ & $19.616(0.057)$ & $18.727(0.032)$ & $18.621(0.035)$ & $18.608(0.185)$ \\
\hline $55192.59 *$ & $\ldots$ & $\ldots$ & $18.910(0.227)$ & & \\
\hline 55192.67 & $\cdots$ & $\cdots$ & $18.612(0.017)$ & $18.530(0.032)$ & $18.617(0.072)$ \\
\hline 55201.78 & $19.878(0.078)$ & $19.363(0.035)$ & $18.454(0.014)$ & $18.376(0.017)$ & $18.446(0.063)$ \\
\hline $55202.60 *$ & $\ldots$ & & $18.827(0.318)$ & & \\
\hline 55202.78 & $\ldots$ & $19.321(0.035)$ & $18.444(0.019)$ & $18.321(0.025)$ & $18.374(0.103)$ \\
\hline $55203.74 *$ & $\ldots$ & $\ldots$ & $18.772(0.056)$ & & \\
\hline 55204.71 & $\ldots$ & $\ldots$ & $18.434(0.011)$ & $18.354(0.013)$ & $18.487(0.035)$ \\
\hline $55207.69 *$ & $\ldots$ & $\ldots$ & $18.613(0.059)$ & & \\
\hline 55207.79 & $19.869(0.056)$ & $\ldots$ & $18.397(0.017)$ & $18.312(0.022)$ & $18.353(0.037)$ \\
\hline 55208.67 & $19.840(0.159)$ & $19.244(0.087)$ & 18.391(0.016) & $18.320(0.034)$ & $18.393(0.108)$ \\
\hline $55209.65^{*}$ & & & $18.600(0.008)$ & & \\
\hline 55209.67 & $19.745(0.029)$ & $19.260(0.014)$ & $18.406(0.009)$ & $18.259(0.012)$ & $18.325(0.082)$ \\
\hline 55221.70 & $20.392(0.141)$ & $19.519(0.051)$ & & $18.437(0.023)$ & $\ldots$ \\
\hline $55429.98^{*}$ & $\ldots$ & $\ldots$ & 21.972(0.189) & $\ldots$ & $\ldots$ \\
\hline $55441.98 *$ & $\ldots$ & $\ldots$ & $21.741(0.012)$ & $\ldots$ & $\ldots$ \\
\hline $55450.96^{*}$ & $\ldots$ & $\ldots$ & $21.706(0.365)$ & $\ldots$ & $\ldots$ \\
\hline $55452.98 *$ & $\ldots$ & $\cdots$ & $22.307(0.458)$ & $\ldots$ & $\ldots$ \\
\hline $55455.96^{*}$ & $\ldots$ & $\ldots$ & $22.103(0.336)$ & $\ldots$ & $\ldots$ \\
\hline $55456.92 *$ & $\ldots$ & $\ldots$ & $21.767(0.323)$ & $\ldots$ & $\ldots$ \\
\hline $55457.97 *$ & $\ldots$ & $\ldots$ & $22.475(0.451)$ & $\ldots$ & $\ldots$ \\
\hline $55459.01 *$ & $\ldots$ & $\ldots$ & $21.998(0.386)$ & $\ldots$ & $\ldots$ \\
\hline $55473.84 *$ & $\ldots$ & $\ldots$ & $21.598(0.345)$ & $\ldots$ & $\ldots$ \\
\hline $55480.90 *$ & $\ldots$ & $\ldots$ & $21.864(0.490)$ & $\ldots$ & $\ldots$ \\
\hline \multirow{2}{*}{\multicolumn{6}{|c|}{$\begin{array}{l}\cdots \quad \text { PTF12gex } \\
\quad \text { PT }\end{array}$}} \\
\hline & & & & & \\
\hline $56085.85^{*}$ & $\ldots$ & $\ldots$ & $21.145(0.343)$ & $\ldots$ & $\ldots$ \\
\hline $56086.93 *$ & $\ldots$ & $\ldots$ & $20.653(0.371)$ & $\ldots$ & $\ldots$ \\
\hline $56087.91 *$ & $\ldots$ & $\ldots$ & $20.811(0.098)$ & $\ldots$ & $\ldots$ \\
\hline $56089.84^{*}$ & $\ldots$ & $\ldots$ & $20.322(0.137)$ & $\ldots$ & $\ldots$ \\
\hline $56091.89 *$ & $\ldots$ & $\ldots$ & $20.462(0.147)$ & $\ldots$ & $\ldots$ \\
\hline $56092.79 *$ & $\ldots$ & $\ldots$ & $20.337(0.080)$ & $\ldots$ & $\ldots$ \\
\hline $56093.88^{*}$ & $\ldots$ & $\ldots$ & $20.416(0.157)$ & $\ldots$ & $\ldots$ \\
\hline $56094.79^{*}$ & $\ldots$ & $\ldots$ & $20.276(0.082)$ & $\ldots$ & $\ldots$ \\
\hline $56096.81 *$ & $\ldots$ & $\ldots$ & $20.273(0.090)$ & $\ldots$ & $\ldots$ \\
\hline $56098.83 *$ & $\ldots$ & $\ldots$ & $20.142(0.072)$ & $\ldots$ & $\ldots$ \\
\hline $56099.74 *$ & $\ldots$ & $\ldots$ & $20.124(0.015)$ & $\ldots$ & $\ldots$ \\
\hline $56100.80^{*}$ & $\ldots$ & $\ldots$ & $20.073(0.085)$ & $\ldots$ & $\ldots$ \\
\hline $56101.73 *$ & $\ldots$ & $\ldots$ & $20.074(0.153)$ & $\ldots$ & $\ldots$ \\
\hline $56102.80^{*}$ & $\ldots$ & $\ldots$ & $20.025(0.062)$ & $\ldots$ & $\ldots$ \\
\hline $56103.73^{*}$ & $\ldots$ & $\ldots$ & $19.962(0.078)$ & $\ldots$ & $\ldots$ \\
\hline $56104.77^{*}$ & $\ldots$ & $\ldots$ & $20.048(0.072)$ & $\ldots$ & $\ldots$ \\
\hline $56105.76^{*}$ & $\ldots$ & $\ldots$ & $19.827(0.058)$ & $\ldots$ & $\ldots$ \\
\hline $56106.75^{*}$ & $\ldots$ & $\ldots$ & $20.050(0.162)$ & $\cdots$ & $\ldots$ \\
\hline $56107.78^{*}$ & $\ldots$ & $\ldots$ & $19.827(0.075)$ & $\ldots$ & $\ldots$ \\
\hline $56108.78 *$ & $\ldots$ & $\ldots$ & $19.732(0.233)$ & $\ldots$ & $\ldots$ \\
\hline $56109.68^{*}$ & $\ldots$ & $\ldots$ & $19.785(0.083)$ & $\ldots$ & $\ldots$ \\
\hline $56111.76^{*}$ & $\ldots$ & $\ldots$ & $19.830(0.147)$ & $\ldots$ & $\ldots$ \\
\hline $56113.79 *$ & $\ldots$ & $\ldots$ & $19.643(0.062)$ & & \\
\hline
\end{tabular}

Notes. Photometric epochs obtained with P48 are marked with “*”, those obtained by LCOGT are marked with “**”. For PTF12kso the epochs marked with "†" correspond to BVRI photometry from MLO, which was standardized to the Johnson-Cousins filter system (no correction has been made to convert the obtained VRI magnitudes to the gri system). The rest of the data were obtained by P60. 
F. Taddia et al.: PTF and CCCP long-rising SNe II

Table A.2. continued.

\begin{tabular}{|c|c|c|c|c|c|}
\hline $\begin{array}{l}\text { JD-2 } 400000 \\
\text { (days) }\end{array}$ & $\begin{array}{c}B \\
(\mathrm{mag})\end{array}$ & $\begin{array}{c}g \\
(\mathrm{mag})\end{array}$ & $\begin{array}{c}r \\
(\mathrm{mag}) \\
\end{array}$ & $\begin{array}{c}i \\
(\mathrm{mag}) \\
\end{array}$ & $\begin{array}{c}z \\
(\mathrm{mag})\end{array}$ \\
\hline $56114.73^{*}$ & $\cdots$ & . & $19.747(0.064)$ & $\ldots$ & . \\
\hline $56115.84^{*}$ & $\ldots$ & $\ldots$ & $19.723(0.045)$ & $\ldots$ & $\ldots$ \\
\hline $56116.74 *$ & $\ldots$ & $\ldots$ & $19.697(0.090)$ & $\ldots$ & $\ldots$ \\
\hline $56117.82 *$ & $\ldots$ & $\ldots$ & $19.658(0.039)$ & $\ldots$ & $\ldots$ \\
\hline $56118.75^{*}$ & $\ldots$ & $\ldots$ & $19.700(0.078)$ & $\ldots$ & $\ldots$ \\
\hline $56119.80^{*}$ & $\ldots$ & $\ldots$ & $19.649(0.065)$ & $\ldots$ & $\ldots$ \\
\hline $56123.78^{*}$ & $\ldots$ & $\ldots$ & $19.611(0.051)$ & $\ldots$ & $\ldots$ \\
\hline $56125.80^{*}$ & $\ldots$ & $\ldots$ & $19.510(0.088)$ & $\ldots$ & $\ldots$ \\
\hline $56126.80^{*}$ & $\ldots$ & $\ldots$ & $19.565(0.049)$ & $\ldots$ & $\ldots$ \\
\hline $56128.78^{*}$ & $\ldots$ & $\ldots$ & $19.556(0.084)$ & $\ldots$ & $\ldots$ \\
\hline $56130.75^{*}$ & $\ldots$ & $\ldots$ & $19.489(0.047)$ & $\ldots$ & $\ldots$ \\
\hline $56131.77 *$ & $\ldots$ & $\ldots$ & $19.413(0.075)$ & $\ldots$ & $\ldots$ \\
\hline $56132.77 *$ & $\ldots$ & $\ldots$ & $19.545(0.045)$ & $\ldots$ & $\ldots$ \\
\hline $56135.73^{*}$ & $\ldots$ & $\ldots$ & $19.513(0.036)$ & $\ldots$ & $\ldots$ \\
\hline 56177.68 & $\ldots$ & $21.343(0.167)$ & $19.754(0.054)$ & $19.281(0.028)$ & $\ldots$ \\
\hline 56178.67 & $\ldots$ & $21.136(0.125)$ & & $\ldots$ & $\ldots$ \\
\hline 56186.65 & $\ldots$ & $\ldots$ & $20.259(0.051)$ & $19.833(0.067)$ & $\ldots$ \\
\hline 56187.68 & $\ldots$ & $\ldots$ & $20.289(0.068)$ & $19.825(0.074)$ & $\ldots$ \\
\hline \multicolumn{6}{|c|}{$\begin{array}{l}{ }^{\prime} \text { PTF12kso } \\
\text { PTo }\end{array}$} \\
\hline $2456198.983 *$ & $\ldots$ & $\ldots$ & $18.406(0.018)$ & $\ldots$ & $\ldots$ \\
\hline $2456228.944 *$ & $\ldots$ & $\ldots$ & $18.041(0.025)$ & $\ldots$ & $\ldots$ \\
\hline $2456238.002 *$ & $\ldots$ & $\ldots$ & $17.974(0.002)$ & $\ldots$ & $\ldots$ \\
\hline 2456238.97 & $\ldots$ & $\ldots$ & $18.020(0.008)$ & $\ldots$ & $\ldots$ \\
\hline $2456239.992 *$ & $\ldots$ & $\ldots$ & $17.984(0.027)$ & $\ldots$ & $\ldots$ \\
\hline $2456240.84 * *$ & $\ldots$ & $18.931(0.028)$ & $18.007(0.013)$ & $17.837(0.038)$ & $\ldots$ \\
\hline $2456241.80 * *$ & $\ldots$ & $19.016(0.047)$ & $18.036(0.060)$ & $17.779(0.067)$ & $\ldots$ \\
\hline 2456245.71 & $19.652(0.060)$ & $18.950(0.024)$ & $17.993(0.014)$ & $17.782(0.012)$ & $\ldots$ \\
\hline $2456245.77 * *$ & $\ldots$ & $19.054(0.036)$ & $18.010(0.048)$ & $17.791(0.046)$ & $17.532(0.020)$ \\
\hline $2456246.81^{* *}$ & $\ldots$ & $18.991(0.018)$ & & $\ldots$ & $\ldots$ \\
\hline $2456247.74 * *$ & $\ldots$ & $19.049(0.151)$ & $17.964(0.023)$ & $17.909(0.029)$ & $17.843(0.093)$ \\
\hline $2456248.78^{* *}$ & $\ldots$ & $19.101(0.060)$ & $18.084(0.044)$ & $17.822(0.059)$ & $17.639(0.122)$ \\
\hline $2456250.77 * *$ & $\ldots$ & $19.135(0.070)$ & $17.937(0.074)$ & $17.807(0.028)$ & $17.539(0.092)$ \\
\hline 2456250.88 & $19.744(0.039)$ & $19.080(0.019)$ & $18.011(0.011)$ & $17.853(0.014)$ & $\ldots$ \\
\hline 2456251.70 & $19.882(0.076)$ & $19.034(0.026)$ & $18.026(0.020)$ & $17.765(0.016)$ & $\ldots$ \\
\hline $2456252.76^{* *}$ & $\ldots$ & $19.130(0.032)$ & $18.066(0.117)$ & $17.813(0.045)$ & $17.640(0.074)$ \\
\hline $2456252.99 \dagger$ & $\ldots$ & & $18.057(0.129)$ & & $\ldots$ \\
\hline $2456253.81^{* *}$ & $\ldots$ & $19.055(0.102)$ & $17.996(0.068)$ & $17.897(0.137)$ & $\ldots$ \\
\hline 2456254.69 & $19.903(0.079)$ & $19.136(0.036)$ & $18.029(0.014)$ & $17.872(0.015)$ & $\ldots$ \\
\hline $2456254.716^{*}$ & $\ldots$ & & $18.000(0.035)$ & & $\ldots$ \\
\hline $2456254.76^{* *}$ & $\ldots$ & $19.109(0.030)$ & $18.018(0.006)$ & $17.867(0.061)$ & $17.748(0.104)$ \\
\hline $2456256.76^{* *}$ & $\ldots$ & $19.176(0.032)$ & $18.144(0.028)$ & $17.863(0.076)$ & $17.672(0.101)$ \\
\hline $2456256.915^{*}$ & $\ldots$ & & $18.033(0.005)$ & & $\ldots$ \\
\hline 2456257.68 & $\ldots$ & $19.036(0.078)$ & $18.047(0.029)$ & $17.875(0.024)$ & $\ldots$ \\
\hline $2456259.76^{* *}$ & $\ldots$ & $19.226(0.104)$ & $18.069(0.063)$ & $17.875(0.123)$ & $17.544(0.083)$ \\
\hline $2456261.75^{* *}$ & $\ldots$ & & $18.140(0.048)$ & $\ldots$ & $\ldots$ \\
\hline $2456264.77 * *$ & $\ldots$ & $19.365(0.112)$ & $18.140(0.100)$ & $17.916(0.015)$ & $17.588(0.081)$ \\
\hline $2456265.73^{* *}$ & $\ldots$ & $19.470(0.110)$ & $18.280(0.120)$ & $18.038(0.124)$ & $17.649(0.064)$ \\
\hline 2456265.76 & $20.179(0.139)$ & $19.433(0.042)$ & $18.233(0.021)$ & $17.970(0.023)$ & $\ldots$ \\
\hline $2456266.73^{* *}$ & .. & $19.509(0.020)$ & $18.303(0.036)$ & $17.938(0.068)$ & $17.799(0.155)$ \\
\hline $2456267.73^{* *}$ & $\ldots$ & $19.666(0.082)$ & $18.349(0.085)$ & $18.041(0.067)$ & $17.873(0.051)$ \\
\hline 2456268.65 & $20.394(0.069)$ & $19.618(0.029)$ & $18.309(0.012)$ & $18.086(0.023)$ & $\ldots$ \\
\hline $2456268.73^{* *}$ & $\ldots$ & $19.623(0.145)$ & $18.491(0.035)$ & $18.032(0.017)$ & $17.834(0.039)$ \\
\hline $2456269.67 * *$ & $\ldots$ & $19.764(0.043)$ & $18.362(0.048)$ & $18.110(0.066)$ & $17.809(0.049)$ \\
\hline $2456270.72 * *$ & $\ldots$ & $19.761(0.075)$ & $18.496(0.055)$ & $18.177(0.064)$ & $17.773(0.146)$ \\
\hline $2456270.88 \dagger$ & $20.401(0.076)$ & $18.897(0.057)$ & $18.203(0.097)$ & $18.250(0.170)$ & $\ldots$ \\
\hline 2456271.67 & $20.628(0.077)$ & $19.910(0.033)$ & $18.467(0.017)$ & $18.183(0.034)$ & $\ldots$ \\
\hline $2456272.72 * *$ & $\ldots$ & $19.928(0.121)$ & $18.516(0.055)$ & $18.222(0.092)$ & $17.847(0.142)$ \\
\hline $2456273.72 * *$ & $\ldots$ & $19.911(0.119)$ & $18.655(0.043)$ & $18.294(0.053)$ & $17.987(0.123)$ \\
\hline $2456274.71 * *$ & $\ldots$ & $19.997(0.075)$ & $18.604(0.010)$ & $18.319(0.102)$ & $17.993(0.075)$ \\
\hline $2456275.67 * *$ & $\ldots$ & $20.160(0.052)$ & $18.816(0.107)$ & $18.393(0.037)$ & $18.091(0.068)$ \\
\hline $2456276.68 * *$ & $\ldots$ & $20.085(0.045)$ & $18.783(0.060)$ & $18.432(0.042)$ & $17.989(0.090)$ \\
\hline $2456277.67 * *$ & $\ldots$ & $20.250(0.080)$ & $18.741(0.039)$ & $18.479(0.069)$ & $18.112(0.101)$ \\
\hline $2456278.67 * *$ & $\ldots$ & $20.215(0.046)$ & $18.825(0.079)$ & $18.470(0.097)$ & $18.250(0.060)$ \\
\hline $2456279.67 * *$ & $\ldots$ & $\ldots$ & $18.869(0.034)$ & $18.554(0.153)$ & $18.186(0.108)$ \\
\hline $2456280.77 * *$ & $\ldots$ & $\ldots$ & $18.922(0.065)$ & $18.693(0.020)$ & $18.283(0.055)$ \\
\hline 2456281.71 & $20.977(0.135)$ & $20.381(0.114)$ & $18.914(0.038)$ & $18.704(0.035)$ & $\ldots$ \\
\hline $2456281.77 * *$ & $\ldots$ & $\ldots$ & $18.939(0.021)$ & $18.592(0.032)$ & .. \\
\hline $2456282.67 * *$ & $\ldots$ & $\ldots$ & $18.931(0.053)$ & $18.617(0.120)$ & $\ldots$ \\
\hline $2456283.67 * *$ & $\ldots$ & $20.349(0.019)$ & $19.008(0.117)$ & $18.810(0.072)$ & $18.233(0.127)$ \\
\hline 2456283.88 & $20.986(0.157)$ & & & $\ldots$ & $\ldots$ \\
\hline $2456284.67 * *$ & $\ldots$ & $20.480(0.093)$ & $19.076(0.093)$ & $18.912(0.038)$ & $18.519(0.099)$ \\
\hline $2456285.72 * *$ & $\ldots$ & $\ldots$ & $18.968(0.151)$ & $18.898(0.109)$ & $18.214(0.000)$ \\
\hline
\end{tabular}


A\&A 588, A5 (2016)

Table A.2. continued.

\begin{tabular}{|c|c|c|c|c|c|}
\hline $\begin{array}{l}\text { JD-2 } 400000 \\
\text { (days) }\end{array}$ & $\begin{array}{c}B \\
(\mathrm{mag}) \\
\end{array}$ & $\begin{array}{c}g \\
(\mathrm{mag})\end{array}$ & $\begin{array}{c}r \\
(\mathrm{mag}) \\
\end{array}$ & $\begin{array}{c}i \\
(\mathrm{mag}) \\
\end{array}$ & $\begin{array}{c}z \\
(\mathrm{mag})\end{array}$ \\
\hline $2456286.72 * *$ & $\cdots$ & $\cdots$ & $19.094(0.109)$ & $18.798(0.135)$ & . \\
\hline $2456287.67 * *$ & $\ldots$ & $\ldots$ & & $18.931(0.172)$ & $\ldots$ \\
\hline $2456288.71 * *$ & $\ldots$ & $\ldots$ & $19.078(0.101)$ & $18.846(0.110)$ & $\ldots$ \\
\hline $2456289.67 * *$ & $\ldots$ & $\ldots$ & $19.240(0.007)$ & $18.986(0.124)$ & $\ldots$ \\
\hline 2456289.86 & $\ldots$ & $\ldots$ & $19.263(0.078)$ & $18.961(0.089)$ & 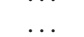 \\
\hline $2456290.67 * *$ & $\ldots$ & $\ldots$ & $19.128(0.073)$ & $18.868(0.105)$ & $\ldots$ \\
\hline $2456291.67 * *$ & $\ldots$ & $\ldots$ & $19.180(0.111)$ & $18.860(0.136)$ & $\ldots$ \\
\hline $2456295.72 * *$ & & & $19.246(0.040)$ & $18.843(0.106)$ & . \\
\hline $2456295.80 \dagger$ & $20.768(0.136)$ & $19.758(0.078)$ & $18.904(0.111)$ & $19.159(0.171)$ & $\ldots$ \\
\hline 2456297.68 ** & $\ldots$ & & & $18.949(0.139)$ & $\ldots$ \\
\hline 2456297.87 & $\ldots$ & $20.889(0.128)$ & & & \\
\hline $2456298.72 * *$ & $\ldots$ & $\ldots$ & $19.250(0.076)$ & $19.008(0.105)$ & $\ldots$ \\
\hline $2456299.68 * *$ & $\cdots$ & $\ldots$ & $19.223(0.064)$ & $19.107(0.038)$ & $\ldots$ \\
\hline 2456300.76 & $21.460(0.179)$ & $\ldots$ & $19.304(0.029)$ & $19.157(0.038)$ & $\ldots$ \\
\hline $2456300.77 * *$ & $\ldots$ & $\ldots$ & $19.256(0.149)$ & $19.124(0.093)$ & $\ldots$ \\
\hline $2456301.68 * *$ & $\ldots$ & $\ldots$ & $19.262(0.059)$ & $19.132(0.064)$ & .. \\
\hline $2456308.63 * *$ & $\ldots$ & $\ldots$ & $19.349(0.050)$ & $19.213(0.099)$ & $\ldots$ \\
\hline $2456309.68 * *$ & $\ldots$ & $\ldots$ & $19.350(0.065)$ & $19.235(0.077)$ & .. \\
\hline $2456310.62 * *$ & $\ldots$ & $\ldots$ & $19.402(0.027)$ & $19.259(0.134)$ & .. \\
\hline $2456311.63 * *$ & $\ldots$ & $\ldots$ & $19.306(0.066)$ & $19.088(0.089)$ & $\ldots$ \\
\hline $2456312.68 * *$ & $\ldots$ & $\ldots$ & $19.384(0.011)$ & $19.385(0.034)$ & $\ldots$ \\
\hline $2456314.57 * *$ & $\ldots$ & $\ldots$ & $19.497(0.016)$ & $19.346(0.108)$ & $\ldots$ \\
\hline $2456315.61 * *$ & $\ldots$ & $\ldots$ & $19.529(0.032)$ & $19.450(0.051)$ & $\ldots$ \\
\hline $2456316.61 * *$ & $\ldots$ & $\ldots$ & $19.405(0.161)$ & $19.228(0.127)$ & .. \\
\hline $2456317.57 * *$ & $\ldots$ & $\ldots$ & $19.516(0.074)$ & $19.354(0.061)$ & $\ldots$ \\
\hline $2456318.61 * *$ & $\ldots$ & $\ldots$ & $19.435(0.071)$ & $19.269(0.115)$ & $\ldots$ \\
\hline 2456321.74 & $\ldots$ & & $19.465(0.110)$ & $19.366(0.102)$ & $\ldots$ \\
\hline 2456322.71 & $\ldots$ & $20.962(0.145)$ & $19.422(0.050)$ & $19.474(0.055)$ & $\ldots$ \\
\hline $2456323.68 * *$ & & $\ldots$ & $19.389(0.029)$ & $19.402(0.014)$ & $\ldots$ \\
\hline 2456323.71 & $21.752(0.171)$ & $\ldots$ & $\ldots$ & $\ldots$ & $\ldots$ \\
\hline 2456324.69 & $21.763(0.191)$ & $\ldots$ & & & $\ldots$ \\
\hline $2456325.57 * *$ & ... & $\ldots$ & $19.500(0.039)$ & $19.425(0.060)$ & $\ldots$ \\
\hline 2456325.69 & $\ldots$ & $21.134(0.155)$ & $19.464(0.119)$ & $19.587(0.172)$ & $\ldots$ \\
\hline $2456326.57 * *$ & $\ldots$ & $\ldots$ & $19.435(0.101)$ & $19.410(0.167)$ & $\ldots$ \\
\hline $2456327.60 * *$ & & & $19.463(0.040)$ & $19.434(0.061)$ & .. \\
\hline 2456327.77 & $21.431(0.185)$ & $21.152(0.112)$ & & & .. \\
\hline $2456328.60 * *$ & & $\ldots$ & $19.358(0.031)$ & $19.301(0.063)$ & $\ldots$ \\
\hline 2456328.68 & $22.014(0.202)$ & $\ldots$ & $19.517(0.028)$ & $19.511(0.038)$ & .. \\
\hline $2456329.64 * *$ & $\ldots$ & $\ldots$ & $19.560(0.016)$ & $19.441(0.053)$ & $\ldots$ \\
\hline $2456330.64 * *$ & $\ldots$ & & $19.491(0.025)$ & $19.379(0.030)$ & $\ldots$ \\
\hline 2456330.68 & $\ldots$ & $21.143(0.086)$ & & & $\ldots$ \\
\hline $2456331.64 * *$ & $\ldots$ & $\ldots$ & $19.563(0.009)$ & $19.531(0.051)$ & $\ldots$ \\
\hline $2456333.60 * *$ & $\ldots$ & $\ldots$ & $19.550(0.059)$ & $19.600(0.030)$ & $\ldots$ \\
\hline $2456334.60 * *$ & & & $19.470(0.036)$ & $19.573(0.021)$ & $\ldots$ \\
\hline 2456335.72 & $21.540(0.191)$ & $21.066(0.121)$ & $19.564(0.053)$ & $19.508(0.053)$ & $\ldots$ \\
\hline $2456338.61 * *$ & $\ldots$ & ... & $19.637(0.001)$ & $19.587(0.001)$ & $\ldots$ \\
\hline $2456339.60 * *$ & $\ldots$ & $\ldots$ & $19.617(0.041)$ & & $\ldots$ \\
\hline $2456340.61 * *$ & $\ldots$ & & $19.584(0.039)$ & $19.644(0.006)$ & $\ldots$ \\
\hline 2456340.70 & $\ldots$ & $21.120(0.127)$ & $19.594(0.055)$ & $19.670(0.057)$ & $\ldots$ \\
\hline $2456341.61 * *$ & $\ldots$ & $\ldots$ & $19.609(0.054)$ & $19.626(0.051)$ & $\ldots$ \\
\hline $2456342.57 * *$ & $\ldots$ & $\ldots$ & $19.543(0.006)$ & $19.685(0.068)$ & $\ldots$ \\
\hline $2456343.57 * *$ & $\ldots$ & $\ldots$ & $19.692(0.091)$ & $19.648(0.040)$ & $\ldots$ \\
\hline $2456344.57 * *$ & $\ldots$ & $\ldots$ & $19.627(0.031)$ & $19.585(0.087)$ & $\ldots$ \\
\hline $2456345.57 * *$ & $\ldots$ & $\ldots$ & $19.695(0.049)$ & $19.616(0.082)$ & $\ldots$ \\
\hline $2456346.57 * *$ & $\ldots$ & $\ldots$ & $19.584(0.058)$ & $19.653(0.041)$ & $\ldots$ \\
\hline 2456346.73 & $\ldots$ & $\ldots$ & $19.635(0.078)$ & $19.741(0.122)$ & $\ldots$ \\
\hline $2456347.56^{* *}$ & $\ldots$ & $\ldots$ & $19.585(0.151)$ & & $\ldots$ \\
\hline $2456348.57 * *$ & $\ldots$ & $\ldots$ & $19.622(0.069)$ & $19.759(0.083)$ & $\ldots$ \\
\hline $2456349.57 * *$ & $\ldots$ & $\ldots$ & $19.716(0.090)$ & $19.662(0.040)$ & $\ldots$ \\
\hline 2456350.67 & $\ldots$ & $\ldots$ & $19.636(0.105)$ & $19.671(0.117)$ & $\ldots$ \\
\hline 2456351.66 & $\ldots$ & $21.409(0.176)$ & & & \\
\hline 2456354.66 & $\ldots$ & & $19.693(0.048)$ & $19.903(0.083)$ & $\ldots$ \\
\hline 2456356.72 & & $21.488(0.15$ & & & \\
\hline 2456363.66 & $21.822(0.170)$ & $21.296(0.161)$ & $19.746(0.044)$ & $19.953(0.059)$ & $\ldots$ \\
\hline 2456364.65 & $21.946(0.168)$ & $\ldots$ & $\ldots$ & $\ldots$ & $\ldots$ \\
\hline 2456366.64 & 21.933(0.199) & & & & \\
\hline 2456367.65 & $21.460(0.142)$ & $\ldots$ & $19.821(0.062)$ & $20.109(0.143)$ & $\ldots$ \\
\hline 2456377.66 & & & $19.795(0.128)$ & & \\
\hline
\end{tabular}


F. Taddia et al.: PTF and CCCP long-rising SNe II

Table A.3. Optical spectroscopy of PTF and CCCP SN 1987A-like SNe and their host galaxies.

\begin{tabular}{|c|c|c|c|c|c|c|}
\hline Date (UT) & $\begin{array}{c}\text { JD-2 } 450000 \\
\text { (days) }\end{array}$ & $\begin{array}{l}\text { Phase }^{a} \\
\text { (days) }\end{array}$ & Target & Telescope & Instrument & $\begin{array}{l}\text { Range } \\
(\AA)\end{array}$ \\
\hline 08 Nov. 2009 & 5143.9 & +6.7 & PTF09gpn & P200 & DBSP & $3357-10207$ \\
\hline 06 Nov. 2012 & 6237.6 & +61.6 & PTF12kso & Copernico $1.82 \mathrm{~m}$ & AFOSC & $4673-7386$ \\
\hline 07 Nov. 2012 & 6238.9 & +62.9 & PTF12kso & P200 & DBSP & $3202-9946$ \\
\hline 08 Jan. 2013 & 6301.8 & +124.8 & PTF12kso & Keck-2 & DEIMOS & $4500-9632$ \\
\hline 27 Sep. 2015 & 7292.5 & $\ldots$ & PTF12kso host galaxy & Keck-2 & DEIMOS & $4650-9500$ \\
\hline 16 Jul. 2012 & 6124.5 & +38.8 & PTF12gcx & Keck-2 & DEIMOS & $4402-9593$ \\
\hline 26 Jul. 2012 & 6134.5 & +48.8 & PTF12gcx & P200 & DBSP & 3502-9992 \\
\hline 23 Aug. 2012 & 6162.7 & +77.0 & PTF12gcx & Lick 3-m & Kast & $3458-10332$ \\
\hline 19 Nov. 2004 & 3328.8 & +78.3 & SN 2004ek & P200 & DBSP & 4000-9776 \\
\hline 14 Dec. 2004 & 3353.5 & +103.0 & SN 2004ek & Keck-1 & LRIS & $3450-9200$ \\
\hline 14 Jan. 2005 & 3385.1 & +133.6 & SN 2004ek & P200 & DBSP & $3300-9900$ \\
\hline 24 Sep. 2004 & 3272.5 & +8.2 & SN 2004em & Lick 3-m & Kast & $3320.00-10500$ \\
\hline 18 Oct. 2004 & 3296.5 & +32.2 & SN 2004em & Keck-1 & LRIS & $3450-9200$ \\
\hline 19 Nov. 2004 & 3328.6 & +65.3 & SN 2004em & P200 & DBSP & $3500-9780$ \\
\hline 05 Dec. 2004 & 3344.6 & +81.3 & SN 2004em & P200 & DBSP & $3500-9796$ \\
\hline 05 Apr. 2005 & 3466.0 & +201.7 & SN 2004em & P200 & DBSP & 3600-9991 \\
\hline 16 Jul. 2005 & 3567.5 & +43.8 & SN 2005ci & P200 & DBSP & $3400-9700$ \\
\hline 14 Aug. 2005 & 3596.7 & +73.0 & SN 2005ci & P200 & DBSP & 3500-9801 \\
\hline
\end{tabular}

Notes. ${ }^{(a)}$ From explosion date.

Table A.4. Relative line fluxes and host-galaxy metallicity measurements.

\begin{tabular}{|c|c|c|c|}
\hline Line & $\begin{array}{c}\text { Flux } \\
\left(F / F_{\mathrm{H} \alpha}\right)\end{array}$ & Method & $\begin{array}{c}12+\log (\mathrm{O} / \mathrm{H}) \\
(\mathrm{dex})\end{array}$ \\
\hline \multicolumn{4}{|c|}{ PTF09gpn host } \\
\hline [O II] $\lambda 3727$ & $0.4093 \pm 0.2367$ & R23 & $7.77 \pm 0.15$ \\
\hline $\mathrm{H} \delta \lambda 4101$ & $0.0507 \pm 0.0259$ & N2 & $8.06 \pm 0.18$ \\
\hline $\mathrm{H} \gamma \lambda 4341$ & $0.1227 \pm 0.0335$ & $\mathrm{O} 3 \mathrm{~N} 2$ & $8.06 \pm 0.14$ \\
\hline [O III] $\lambda 4363$ & $0.0487 \pm 0.0186$ & & \\
\hline $\mathrm{H} \beta \lambda 4861$ & $0.3683 \pm 0.0244$ & & \\
\hline [O III] $\lambda 4959$ & $0.4341 \pm 0.0703$ & & \\
\hline [O III] $\lambda 5007$ & $1.2848 \pm 0.0249$ & & \\
\hline Н $\alpha \lambda 6563$ & $1.0000 \pm 0.0192^{*}$ & & \\
\hline [N II] $\lambda 6584$ & $0.0289 \pm 0.0113$ & & \\
\hline [S II] $\lambda 6717$ & $0.1225 \pm 0.0140$ & & \\
\hline [S II] $\lambda 6731$ & $0.0547 \pm 0.0178$ & & \\
\hline \multicolumn{4}{|c|}{ PTF12gex host } \\
\hline [O II] $\lambda 3727$ & $2.0219 \pm 0.0111$ & $\mathrm{R} 23$ & $8.27 \pm 0.15$ \\
\hline $\mathrm{H} \gamma \lambda 4341$ & $0.1198 \pm 0.0075$ & $\mathrm{~N} 2$ & $8.52 \pm 0.18$ \\
\hline $\mathrm{H} \beta \lambda 4861$ & $0.3485 \pm 0.0055$ & $\mathrm{O} 3 \mathrm{~N} 2$ & $8.56 \pm 0.14$ \\
\hline [O III] $\lambda 4959$ & $0.0883 \pm 0.0093$ & & \\
\hline [O III] $\lambda 5007$ & $0.2860 \pm 0.0073$ & & \\
\hline [N II] $\lambda 6548$ & $0.083 \pm 0.0099$ & & \\
\hline $\mathrm{H} \alpha \lambda 6563$ & $1.0000 \pm 0.0089^{\#}$ & & \\
\hline [N II] $\lambda 6584$ & $0.2408 \pm 0.0041$ & & \\
\hline [S II] $\lambda 6717$ & $0.2166 \pm 0.0062$ & & \\
\hline [S II] $\lambda 6731$ & $0.1590 \pm 0.0079$ & & \\
\hline \multicolumn{4}{|c|}{ PTF12kso host } \\
\hline [O III] $\lambda 4959$ & $0.2429 \pm 0.0256$ & N2 & $\leq 8.04 \pm 0.18$ \\
\hline $\mathrm{H} \alpha \lambda 6563$ & $1.0000 \pm 0.0028^{* *}$ & & \\
\hline$[\mathrm{N} \mathrm{II}] \lambda 6584$ & $\leq 0.0257$ & & \\
\hline
\end{tabular}

Notes. ${ }^{(*)} \mathrm{H} \alpha$ flux is $2.42 \times 10^{-14} \mathrm{erg} \mathrm{s}^{-1} \mathrm{~cm}^{-2}$. ${ }^{(\#)} \mathrm{H} \alpha$ flux is $6.72 \times 10^{-14} \mathrm{erg} \mathrm{s}^{-1} \mathrm{~cm}^{-2}$. (**) $\mathrm{H} \alpha$ flux is $1.36 \times 10^{-15} \mathrm{erg} \mathrm{s}^{-1} \mathrm{~cm}^{-2}$. 